\title{
Fast, Three-Dimensional Fluorescence Imaging of Living Cells
}

Doctoral Dissertation

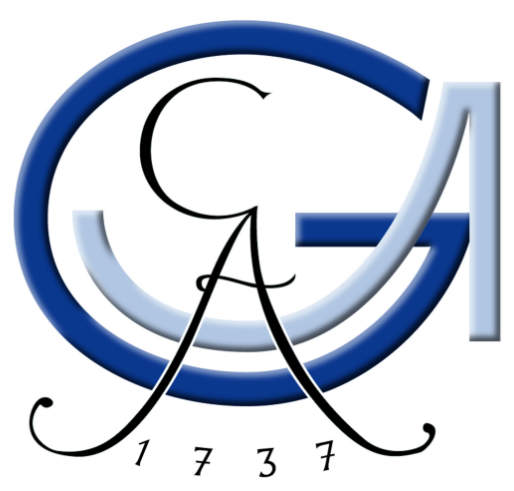

by

HONGJE JANG

III. Institute of Physics - Biophysics

GEORG-August-UNIVERSITY GÖTTINGEN

OCTOBER 2021 



\title{
Fast, Three-Dimensional Fluorescence Imaging of Living Cells
}

\author{
Dissertation \\ for the award of the degree \\ 'Doctor rerum naturalium' \\ of the Georg-August-Universität Göttingen \\ within the doctoral program in Physics \\ of the Georg-August University School of Science (GAUSS)
}

SUBMITTED BY

Hongje Jang

FROM BUSAN, SOUTH KOREA

GÖTTINGEN, OCTOBER 2021 
Thesis ADVISORY COMMITTEE

Prof Dr. Jörg Enderlein

Drittes Physikalisches Institut, Georg-August-Universität Göttingen

Prof Dr. Andreas Janshoff

Institut für Physikalische Chemie, Georg-August-Universität Göttingen

(apl.) Prof. Dr. Alexander Egner

Laser-Laboratorium Göttingen

MEMBERS OF THE EXAMINATION BOARD

\section{Referee: Prof Dr. Jörg Enderlein}

Drittes Physikalisches Institut, Georg-August-Universität Göttingen

Co-referee: (apl.) Prof. Dr. Alexander Egner

Laser-Laboratorium Göttingen

OTHER EXAMINATION BOARD

Prof. Dr. Andreas Janshoff

Institut für Physikalische Chemie, Georg-August-Universität Göttingen

Prof. Dr. Timo Betz

Drittes Physikalisches Institut, Georg-August-Universität Göttingen

Prof. Dr. Sarah Köster

Institut für Röntgenphysik, Georg-August-Universität Göttingen

Prof. Dr. Simone Techert

Institut für Röntgenphysik, Georg-August-Universität Göttingen

DATE OF THE ORAL EXAM: 13. DECEMBER. 2021 


\section{AFFidavit}

Hereby, I declare that the presented thesis has been written independently and with no other sources and aids than quoted. 



\section{TABle of Contents}

Page

$\begin{array}{ll}\text { Abstract } & \text { xi }\end{array}$

1 Introduction 1

1.1 Description of modern fluorescence microscopy methods in comparison to wide-field microscopy . . . . . . . . . . . . . . . . . . . . . 1

1.2 Deconvolution methods to improve wide-field microscopy data . . . . . . 6

1.3 3D-SUPPOSe and multi-plane wide-field microscopy for live-cell fluorescence 9

2 Theory 13

2.1 Multi-plane Wide-field Fluorescence Microscopy . . . . . . . . . . . . . . . 13

2.1 .1 Fluorescence . . . . . . . . . . . . . . . . . . . . 13

2.1.2 Illuminators and filters to isolate specific wavelengths . . . . . . 15

2.1 .3 Lenses . . . . . . . . . . . . . . . . . . . . 18

2.1.4 Point Spread Function (PSF) . . . . . . . . . . . . . . . 21

2.1.5 Multi-plane Wide-field Microscopy . . . . . . . . . . . . . . . . 22

2.2 Digital Image Processing . . . . . . . . . . . . . . . . . . . . . . . 25

2.2.1 Image sensor and sampling . . . . . . . . . . . . . 26

2.2.2 Convolution and Image Kernel . . . . . . . . . . . . . . . . 27

2.2 .3 Noise . . . . . . . . . . . . . . . . . . . . . . 29

2.2.4 Deconvolution as an Ill-posed Inverse Problem . . . . . . . . . . 30

2.3 Mathematical Tools to Solve the Inverse Problem . . . . . . . . . . . . . . 31

2.3.1 Regression Analysis . . . . . . . . . . . . . . . . . 31

2.3 .2 Penalized Regression . . . . . . . . . . . . . . . . . 32

2.3.3 Optimization Method . . . . . . . . . . . . . . 34

2.4 Image Deconvolution . . . . . . . . . . . . . . . . . . . . . . . 36

2.4 .1 Lucy-Richardson . . . . . . . . . . . . . . . . . 37 
2.4 .2 ThreeDeconv . . . . . . . . . . . . . . . . 38

2.4 .3 Sparse Deconvolution . . . . . . . . . . . . . . . 40

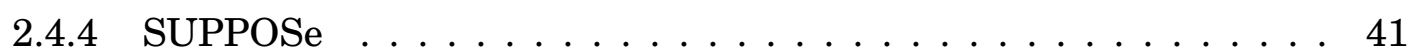

2.5 Analysis methods for fluorescence microscopy . . . . . . . . . . . . . 43

2.5.1 Center of fluorescence mass and geometric center . . . . . . . . 43

2.5.2 3D Optical Flow Estimation . . . . . . . . . . . . . . . 45

3 Image analysis methods for multi-plane microscopy 49

3.1 Method ......................... 50

3.1.1 Calibration of the Multi-plane Setup . . . . . . . . . . . . . 50

3.1.2 Intensity Calibration of the Individual Imaging Planes . . . . . . 51

3.1.3 Deconvolution Method by Superposition of Dispersed Points (3DSUPPOSe . . . . . . . . . . . . . . . . . . 54

3.1.4 New 3D Optical Flow Estimation . . . . . . . . . . . . . . . 57

3.2 Results . . . . . . . . . . . . . . . . . . . . . 60

3.2.1 Intensity Profile of Standard Samples _ . . . . . . . . . . . . . 60

3.2.2 Deconvolution Results - 3D-SUPPOSe . . . . . . . . . . . . . 65

3.2.3 Combination of Multi-plane and 3D-SUPPOSe . . . . . . . . 70

3.2 .4 3D Bead Flow Analysis . . . . . . . . . . . . . . . . 73

3.3 Discussion . . . . . . . . . . . . . . . . . . . . 74

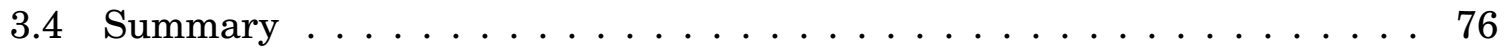

4 Live Cell Imaging - Cardiomyocyte 79

4.1 Method ......................... 81

4.1.1 Sample Preparation . . . . . . . . . . . . . . 81

4.1 .2 Imaging and image processing . . . . . . . . . . . . . 81

4.2 Results . . . . . . . . . . . . . . . . . . . . 82

4.2.1 Planar Beating Motion of Single Live Cardiomyocyte . . . . . . . . 82

4.2.2 3D Movement of $\alpha$-ACTN-2 in Single Z-discs . . . . . . . . . . . 86

4.3 Discussion . . . . . . . . . . . . . . . . . . . 88

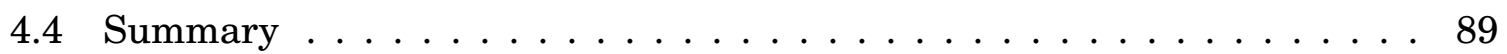

5 Live Cell Imaging - Dictyostelium Discoideum 91

5.1 Method .............................. 92

5.1 .1 Cell Preparation . . . . . . . . . . . . . . . . . 92

5.1 .2 Microscope . . . . . . . . . . . . . . . . . 93 
5.1 .3 Cell Flattening Device . . . . . . . . . . . . . . . . . . 93

5.2 Results . . . . . . . . . . . . . . . . . . 96

5.2.1 Height Profile - Cell Flattening Effect . . . . . . . . . . . . . . . 96

5.2 .2 Evaluation of Effect on Cell Vitality . . . . . . . . . . . . . . 98

5.2.3 Temporal Relation between Actin Movement and Geometric Center of a Cell . . . . . . . . . . . . . . . . . . . . . . . 99

5.3 Discussion . . . . . . . . . . . . . . . . . 105

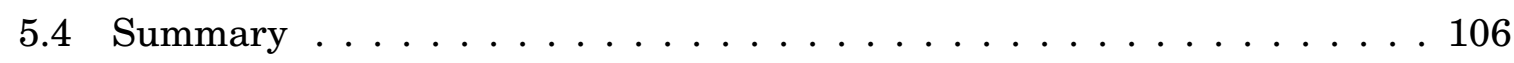

$\begin{array}{llr}6 & \text { Conclusion } & 107\end{array}$

$\begin{array}{ll}\text { Bibliography } & 111\end{array}$

$\begin{array}{ll}\text { Acknowledgements } & 123\end{array}$ 


\section{List OF FiguRES}

1.1 Scheme of stimulated emission depletion microscopy (STED) . . . . . . . . 3

1.2 Image of strucured illumination microscopy $\ldots \ldots \ldots \ldots$

1.3 Schematics of single-molecule localization microscopy . . . . . . . . . . 4

2.1 Jablonski diagram . . . . . . . . . . . . . . . . . . . . 14

2.2 Absorption and emission spectra . . . . . . . . . . . . . 15

2.3 Spectra of different types of light sources . . . . . . . . . . . . . . . 17

2.4 Thin lens . . . . . . . . . . . . . . . . . . . . . 19

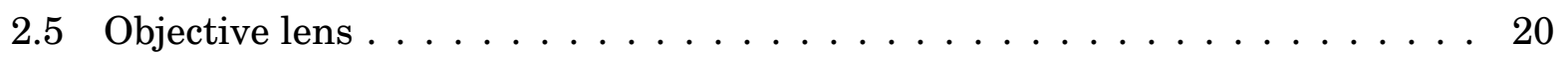

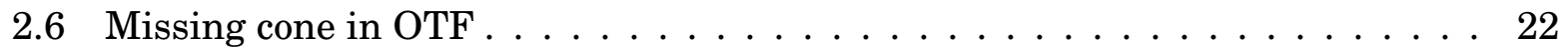

2.7 bi-focal image scheme . . . . . . . . . . . . . . . . . 23

2.8 Multi-plane setup . . . . . . . . . . . . . . . . . . . 24

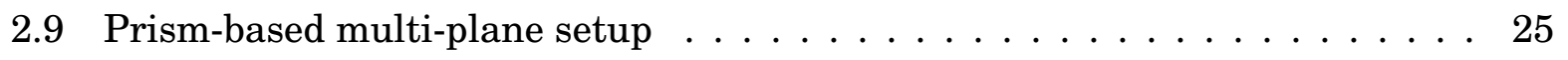

2.10 Digital image sampling process . . . . . . . . . . . . . . . . . 26

2.11 Examples of image kernels . . . . . . . . . . . . . . . . . . 28

2.12 Effect of PSF and noise on an image . . . . . . . . . . . . . 30

2.13 Overfitting example . . . . . . . . . . . . . . . . . . 32

2.14 Ridge and Lasso regression . . . . . . . . . . . . . . . . . . . 34

2.15 Deconvolution result using ThreeDeconv . . . . . . . . . . . . . . . 38

2.16 Sparse deconvolution . . . . . . . . . . . . . . . . . . 41

2.17 Deconvolution result of SUPPOSe . . . . . . . . . . . . . . . . 43

2.18 Flow estimation using PatchMatch method $\ldots \ldots \ldots \ldots$. . . . . . . 47

3.1 Calibration method for multi-plane setup . . . . . . . . . . . . . . 51

3.2 New brightness calibration scheme for multi-plane setup . . . . . . . . . . 53

3.3 Deconvolution scheme of 3 D-SUPPOSe $\ldots \ldots \ldots \ldots \ldots \ldots$

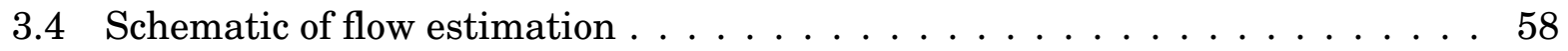


3.5 Relative intensity of image planes . . . . . . . . . . . . . . . 61

3.6 ArgoSIM image: Comparison of calibration results . . . . . . . . . . . . 64

3.7 ArgoSIM image: 3D rendering results of two different calibration methods . . 64

3.8 Bead images with different deconvolution methods . . . . . . . . . . . 66

3.9 Interpolated bead images with different deconvolution methods $\ldots . \ldots . . .69$

3.10 Resolution enhancement of an ArgoSIM pattern 'I' . . . . . . . . . . . . . 72

3.11 Flow estimation result $\ldots \ldots \ldots \ldots \ldots \ldots$. . . . . . . . . . . . . . . .

4.1 Sarcomere structure . . . . . . . . . . . . . . . . . . . . 80

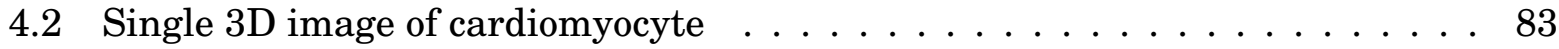

$4.33 \mathrm{D}$ images of cardiomyocyte over time . . . . . . . . . . . . . . . 84

4.4 Covariance graph of cardiomyocyte movies . . . . . . . . . . . . . 85

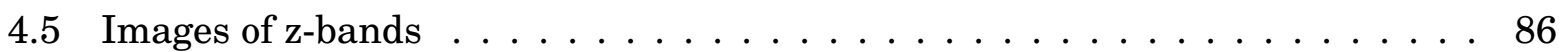

4.6 Analysis result of single Z-disc position $\ldots \ldots \ldots \ldots \ldots$

5.1 Design of the double layered cell flattening device . . . . . . . . . . . . . . . 94

5.2 Modified design of cell flattening device . . . . . . . . . . . . . . . . 95

5.3 A brief description of the making process . . . . . . . . . . . . . . 95

5.4 Change of the height and shape of cells $\ldots \ldots \ldots \ldots$. . . . . . . . 97

$5.53 \mathrm{D}$ rendering result of dicty cells $\ldots \ldots \ldots \ldots \ldots$

5.6 Activity of pressed cells . . . . . . . . . . . . . . . . . . 98

$5.73 \mathrm{D}$ rendering result of the dicty image and actin movement . . . . . . . . 100

5.8 plots of center and actin movement . . . . . . . . . . . . . . . . 101

5.9 Vector plot of actin flow $-1 \ldots \ldots \ldots$. . . . . . . . . . . . . . . . . . . . . . . .

5.10 Vector plot of actin flow $-2 \ldots \ldots \ldots$. . . . . . . . . . . . . 104 



\section{Abstract}

7 his thesis focuses on multi-plane fluorescence microscopy for fast live-cell imaging. To I improve the performance of multi-plane microscopy, I developed new image analysis methods. I used these methods to measure and analyze the movements of cardiomyocytes and Dictyostelium discoideum cells. The multi-plane setup is based on a conventional wide-field microscope using a custom multiple beam-splitter in the detection path. This prism creates separate images of eight distinct focal planes in the sample. Since 3D volume is imaged without scanning, three-dimensional imaging at a very high speed becomes possible. However, as in conventional wide-field microscopy, the "missing cone" of spatial frequencies along the optical axis in the optical transfer function (OTF) prevents optical sectioning in such a microscope. This is in stark contrast to other truly threedimensional imaging modalities like confocal and light-sheet microscopy.

In order to overcome the lack of optical sectioning, I developed a new deconvolution method. Deconvolution describes methods that restore or sharpen an image based on physical assumptions and knowledge of the imaging process. Deconvolution methods have been widely used to sharpen images of microscopes and telescopes. The recently developed SUPPOSe algorithm is a deconvolution algorithm that uses a set of numerous virtual point sources. It tries to reconstruct an image by distributing these point sources in space and optimizing their positions so that the resulting image reproduces as good as possible the measured data. SUPPOSe has never been used for 3D images. Compared to other algorithms, this method has superior performance when the number of pixels is increased by interpolation. In this work, I extended the method to work also with 3D image data. The 3D-SUPPOSe program is suitable for analyzing data of our multi-plane setup. The multi-plane setup has only eight vertically aligned image planes. Furthermore, for accurate reconstruction of 3D images, I studied a method of correcting each image plane's relative brightness constituting an image, and I also developed a method of measuring the movement of point emitters in $3 \mathrm{D}$ space.

Using these methods, I measured and analyzed the beating motion of cardiomyocytes and the chemotaxis of Dicyosteilium discoidem. Cardiomyocytes are the cells of the heart muscle and consist of repetitive sarcomeres. These cells are characterized by fast and periodic movements, and so far the dynamics of these cells was studied only with two-dimensional imaging. In this thesis, the beating motion was analyzed by tracing the spatial distribution of the so-called z-discs, one of the constituent components of 
cardiomyocytes. I found that the vertical distribution of $\alpha$-actinine- 2 in a single $\mathrm{z}$-disc changed very rapidly, which may serve as a starting point for a better understanding the motion of cardiomyocytes.

Dictyostelium discoideum is a well established single cell model organism that migrates along the gradient of a chemoattractant. One has conducted much research to understand the mechanism of chemotaxis, and many efforts have been made to understand the role of actin in the chemotactic motion. By suppressing the motor protein, myosin, a cell line was created that prevented the formation of normal actin filaments. In these myosin null cells, F-actin moves in a flow-like behaviour and induces cell movement. In this study, I imaged the actin dynamics, and I analyzed the flow using the newly created deconvolution and flow estimation methods. As a result of the analysis, the spatio-temporal correlation between pseudo-pod formation and dynamics and actin flow was investigated. 


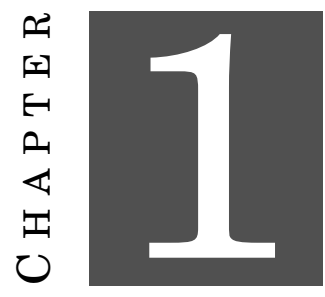

\section{INTRODUCTION}

\subsection{Description of modern fluorescence microscopy methods in comparison to wide-field microscopy}

luorescence microscopy, a measurement technique commonly used in various fields
such as chemistry, biology, and geology, has the ability to selectively measure specific
molecules. A fluorescence microscope generates an image by detecting a fluorescence
signal, which is a photon emitted from an electronically excited molecule. Fluores-
cence is a phenomenon in which photons are emitted after the energetic excitation of
molecules. Molecules that can emit fluorescence signal are called fluorophores or fluores-
cent molecules, and when a target molecule is labeled with this fluorophore, its spatial
distribution can be selectively measured. Therefore, this microscopy technique is widely
used in biological studies to observe the distribution of fluorophore-labeled biomolecules
in vitro and in vivo. In fluorescence microscopy, resolution is one of the main factors that evaluate the performance of the microscope and the quality of measured images. Resolution is the ability of a measurement system to detect and accurately display small changes in an image. In fluorescence microscopy, resolution often refers to spatial resolution, which means the minimum resolvable distance between two molecules. The Abbe diffraction limit, which describes the spatial resolution, $\Delta x$, of general optical systems, including 
microscopes, is defined as[18]

$$
\Delta x=\frac{\lambda}{2 N A}
$$

where $\lambda$ is the emission wavelength and $N A$ is the numerical aperture representing the ability of the lens to collect light. According to the equation, $\Delta x$ becomes larger as the wavelength increases, and spatial resolution decreases. Therefore, spatial resolution is determined by the wavelength of light used and the performance of the microscope.

Another type of resolution to be considered in fluorescence microscopy is temporal resolution. Temporal resolution is highly dependent on the data acquisition rate and refers to the smallest unit of time in which a microscope can detect a change. A high spatial resolution can report about the exact distribution of molecules, and a high temporal resolution can accurately visualize the fast motion of an object. However, there is a trade-off between the two types of resolutions. For example, if we increase the acquisition time to reduce noise and to get a sharper image, the temporal resolution is lowered.

Efforts to improve the spatial resolution of fluorescence microscopy are ongoing. In 1978, C. Cremer and T. Cremer proposed the idea of a complete hologram, so-called 4Pi hologram, which conveys full-field information about the emission of a point source in all directions.[25] After that, in 1994, S. Hell et. al implemented the first 4Pi microscopy system and super-resolution microscopy, Stimulated Emission Depletion Microscopy (STED).[52, 53] Since then, various super-resolution microscopy techniques have been developed, such as Structured Illumination Microscopy (SIM) and single molecule localization microscopy (SMLM).[16, 25, 31, 49, 53, 81, 93]

STED, one of the representative super-resolution microscopy techniques, is a microscopy technique that uses two laser pulses. One pulse electronically excites the fluorophores in the whole volume of illumination, and the STED pulse induces stimulated emission and de-excitation of fluorophores, leaving only a portion of the volume in which excited molecules are distributed. By de-excitation using STED pulses leaving only a part of excited molecules, the volume in which the fluorescence signal is measured at the focal point can be minimized, so that the spatial resolution in a STED microscope is improved (see figure. 1.1). 

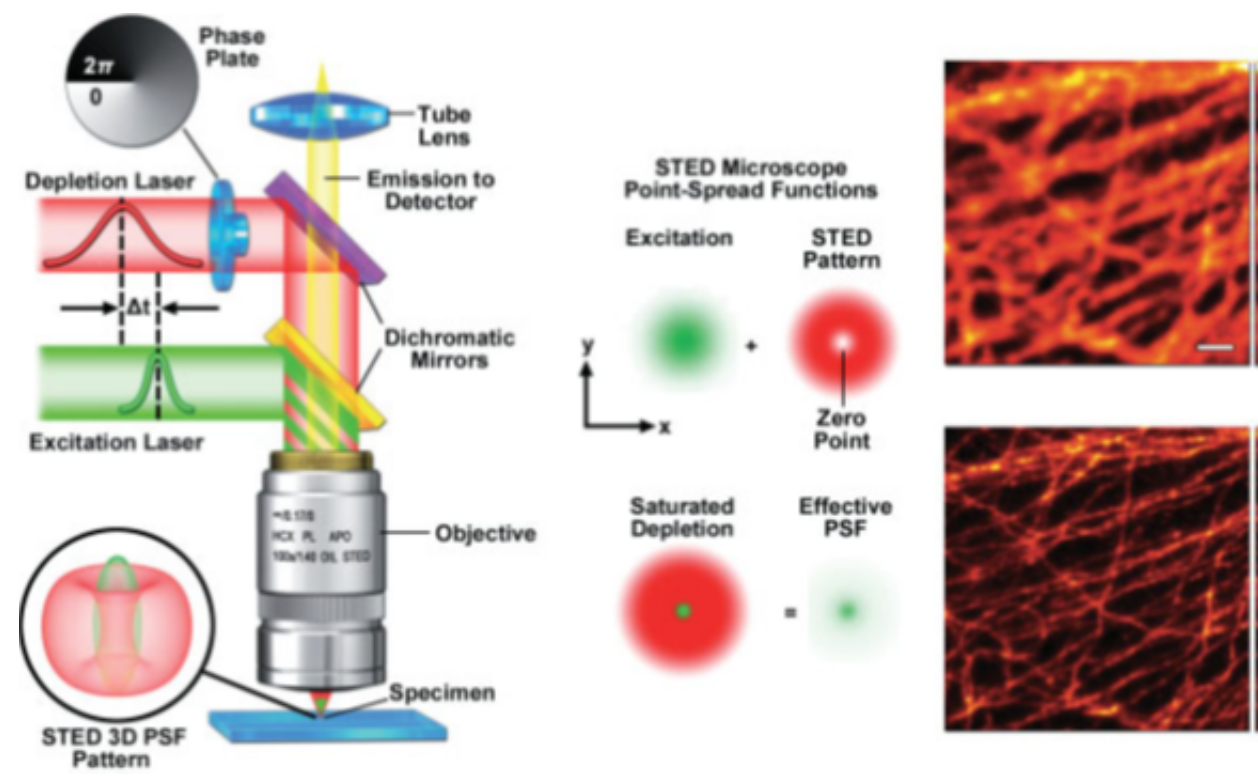

Figure 1.1: Scheme of stimulated emission depletion microscopy (STED) and point spread function (PSF). (a) STED microscopy optical setup showing excitation and depletion lasers, spiral phase plate, and 3D shape of STED PSF. (b) STED PSF. By controlling the zero point area, one can reduce the effective size of the PSF. (c) Widefield image of microtubules stained with Alexa Fluor 594 (d) imaged with the STED system.[79]

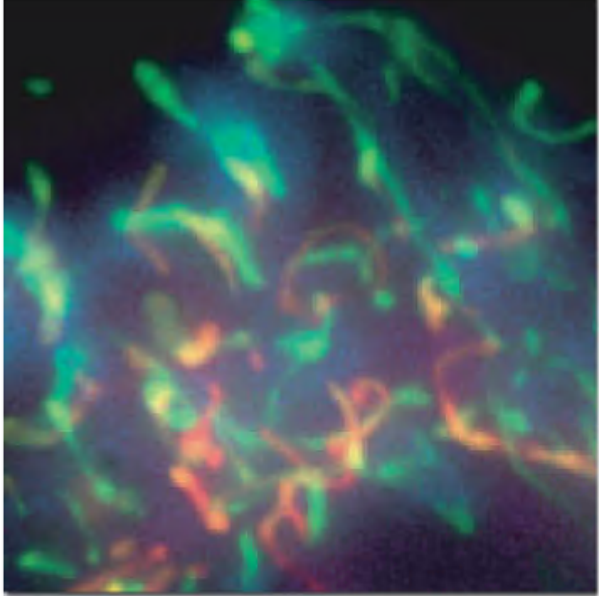

(a)

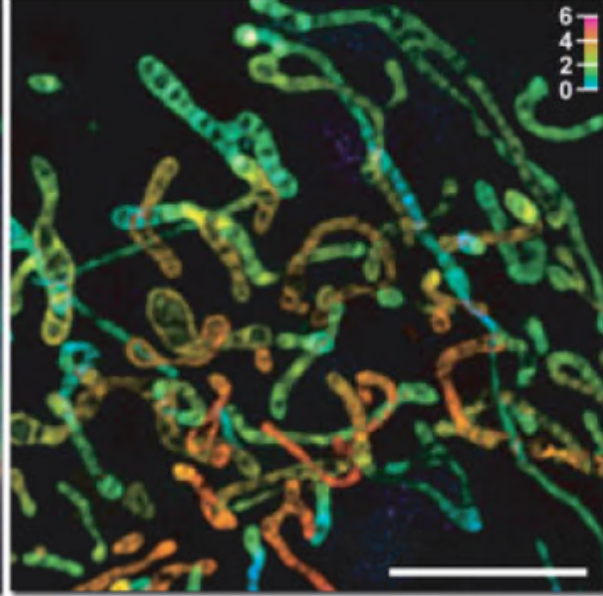

(b)

Figure 1.2: Images of living HeLa cells stained with MitoTracker Green (Life Technologies) (a) Wide-field fluorescence and (b) 3D SIM imaging. The SIM image is a maximum intensity projection along 38 image planes through the cell, while the wide-field image is displayed as a single exposure. The $\mathrm{z}$-depth $(\mu \mathrm{m})$ of the SIM image is color-coded as indicated by the scale bar (height above the cover slip surface in $\mu \mathrm{m}$ ). The SIM result clearly shows details of the mitochondrial matrix and cristae. Bar $=5 \mu \mathrm{m}$.[79] 


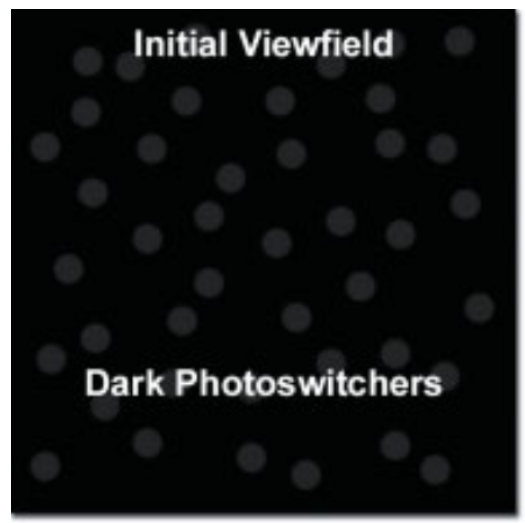

(a)

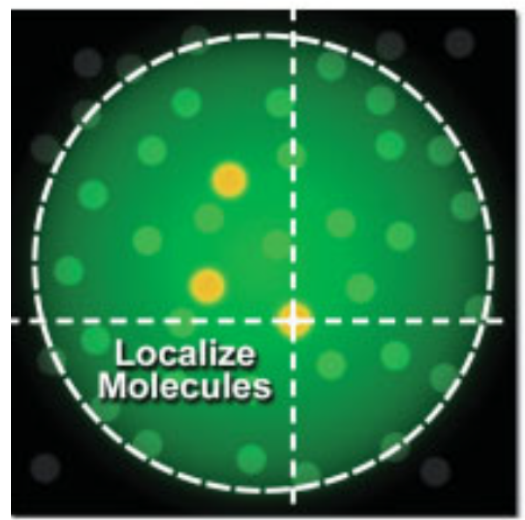

(d)

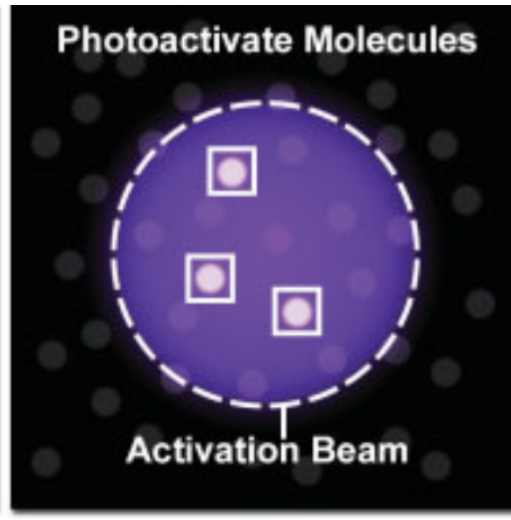

(b)

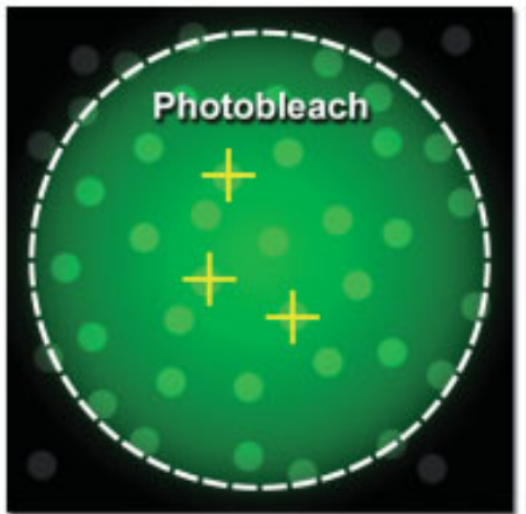

(e)

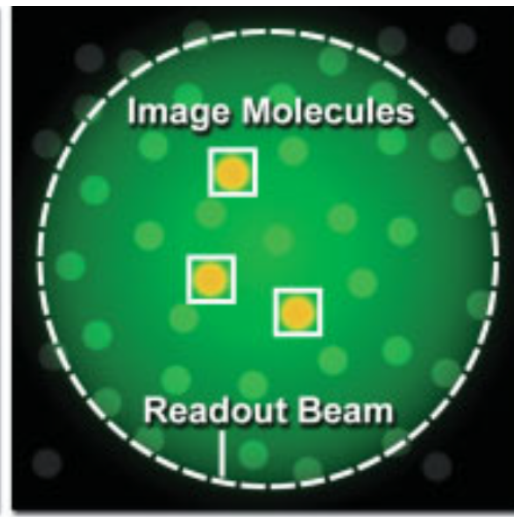

(c)

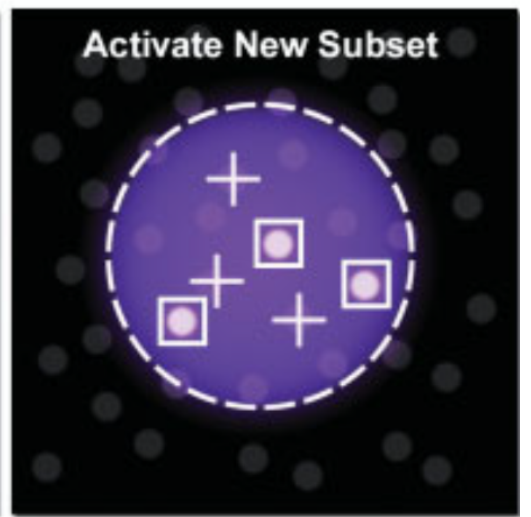

(f)

Figure 1.3: Schematics of single molecule localization microscopy. (a) A densely labeled structural network labeled with a photo-switcher in a dark or inactive state. (b) A sparse set of non-overlapping (boxed circles) fluorescent probes is activated by near ultraviolet. (c) Activated molecules are imaged with a readout laser (green beam) and an EMCCD camera. (d) Sufficient photons are collected from each photoactive molecule to localize their position with high precision. (e) The active molecule is photoconverted or photobleached into a dark state. (f) The process is restarted and continues until all fluorescent probes are exhausted due to photobleaching or the background fluorescence is too high. The final super-resolution image is constructed by plotting the measured positions of the localized fluorescent probes. [79]

Next, SIM is one of the super-resolution microscopy techniques with good compatibility for various biological applications. In 1995, Guerra et al. published the first results of SIM.[49] In SIM, a sample is illuminated with light in a periodic pattern, usually in a sinusoidal pattern along one direction. By taking images at many different phases and orientations of the structured light, one obtains an image set. From the image set, one can compute an super-resolved image. Since SIM does not use any special condition or 
spectroscopic characteristics of fluorophores but the patterned illumination to improve spatial resolution, the technique has the advantage that there are no restrictions on the type of fluorescent molecule or labeling protocol (see figure. 1.2).

Another representative super-resolution microscopy technique is SMLM. In SMLM, such as photoactivated localization microscopy (PALM) and stochastic optical reconstruction microscopy (STORM), molecules are localized with an accuracy of several nanometers. Every single molecule in the image is localized with high precision. After localizing the positions of numerous single emitters, a super-resolved image is reconstructed by synthesizing the location of each emitter. In this process, if several fluorophores are placed in an adjacent position, localization precision is lowered due to signal interference between each other. Therefore, in order to enhance the localization precision of every single emitter, the concentration of emitters generating a signal is controlled by using several methods such as controlling the blinking of a fluorophore by illumination or chemical reaction. Although resolutions down to $1 \mathrm{~nm}$ are theoretically possible with these methods, their actual observed resolution is typically $20 \sim 50 \mathrm{~nm}$, which is much better than Abbe's diffraction limit $(\sim 200 \mathrm{~nm})$ (see figure 1.3).

One can apply super-resolution fluorescence microscopy to determine the nanoscopic molecular distribution in living cells. The improved spatial resolution of fluorescence microscopy by these super-resolution microscopy techniques has expanded the biological application range of fluorescence microscopy. However, its temporal resolution is not sufficient to describe some of fast three-dimensional molecular distributions in living cells. Although the acquisition rate of high-resolution three-dimensional images is still near $1 \mathrm{~Hz}$ range, the movement of molecules in cells is much faster than this rate. For example, the diffusion constant of a typical protein molecule is about $1-100 \mu \mathrm{m}^{2} / \mathrm{s}$ [76], and it moves several micrometers per second. However, even light-sheet microscopy, a three-dimensional microscopy technique that achieves higher temporal resolution than confocal microscopy, is difficult to achieve more than 1-2 frames per second.[114] That is, the protein can move several micrometers while one image is taken. This distance is much larger than the spatial resolution achieved by the super-resolution techniques. Therefore, it is necessary to increase the temporal resolution along with the spatial resolution for precise measurement using a fluorescence microscope.

In order to understand the motion of living cells, a high-resolution three-dimensional fluorescence microscope is important. One good example is a cardiomyocyte. Cardiomy- 
ocytes, which form the cardiac muscle tissue, undergo periodic contractions at a rate of about $1 \mathrm{~Hz}$. Reaction mechanisms of myosin and actin filament, which are representative proteins involved in cardiac motion, are already revealed, but various other proteins are also involved in cell function and contraction. A fast microscope is needed to study the distribution and motion of these molecules involved in cell movement. In particular, the imaging rate should be faster than $1 \mathrm{~Hz}$, since a sufficient number of images is required for each contraction cycle to analyze repetitive patterns. In $2 \mathrm{D}$ studies of a normal cardiomyocyte motion, the motion was measured at a speed of about 10 times faster than the cardiomyocyte's beating rate to describe the natural movement.[8] The study of these objects requires a microscope with particularly excellent temporal resolution.

Wide-field fluorescence microscopy has high temporal resolution, so this technique can allow us to study biological objects like cardiomyocytes. Wide-field fluorescence microscopy is a technique to acquire fluorescence images by illuminating the entire sample volume at once. By illuminating the whole field-of-view at once without scanning, frame rates can go up to hundreds of $\mathrm{Hz}$. Therefore, wide-field microscopes are suitable for studying the rapid movement of biological objects.

However, a wide-field fluorescence microscope has a lower spatial resolution than the super-resolution microscopes described above. Even this microscopy technique has a problem that an image of a focal plane contains also contributions from signals coming from outside the focal plane. Confocal microscopy, a type of fluorescence microscopy commonly used, can generate a clear image of a selected focal plane within a sample through optical sectioning that blocks signals coming from outside the focal plane. However, wide-field microscopes are not designed to block signals from outside the focal plane. Therefore, the wide-field fluorescence microscopy image cannot resolve structural details along the optical axis, which is the propagation direction of the illumination beam.

\subsection{Deconvolution methods to improve wide-field microscopy data}

Deconvolution method allows us to overcome this limitation of wide-field microscopes. Deconvolution is a method of sharpening an image using computational methods. This is done by trying to reverse the convolution of the source distribution with the point spread 
function that constitutes an image. A first computational method for this purpose was developed in the 1940s by Nobert Wiener.

The Wiener filter was developed for the purpose of restoring convoluted signals. In the frequency domain of a digital signal, deconvolution can be expressed in the form of a simple division. Nobert Wiener considered the presence of additional noise when developing his deconvolution procedure. As a result, it was possible to restore a clear signal without noise. After the development of the Wiener filter, this idea was developed into various forms such as the Richardson-Lucy method using an iterative method.[67, 91, 116] In 1984, Agard and Sedat applied this deconvolution method for the first time to reconstruct the 3D structure of a single chromosome.[7] After this first application, the method was then applied widely for 3D microscopy.

Recent advancement in computational techniques have resulted in the development of several new deconvolution algorithms and improvements of existing algorithms.[50, $59,73]$ In 2018, Hayato et al. introduced ThreeDeconv, a deconvolution program for 3D image reconstruction. This program was designed to get images by deconvolution of noisy fluorescent images. During the deconvolution, the noise impact on results is suppressed by the equations that describe the characteristics of noise. By constraining the variation between neighouring pixels, unphysical brightness variations are suppressed. This method was used for image analysis of wide-field microscopy data to eliminate noise and soften the missing cone problem.

Deconvolution has not been used only for the analysis of wide-field microscopic image analysis, but also for other microscopic images like SMLM datasets. In the initial analysis of localization microscopy data, the position of each molecule was fitted using a Gaussian function. However, when several fluorophores are simultaneously excited at close-by positions, the method cannot correctly determine the positions of individual molecules. If the fluorophores are located too close to each other, it is difficult to precisely localize them, since the signals of each fluorophore overlap with each other. Therefore, in SMLM, methods to excite each fluorophore separately are used. Since a single image can only contain a small number of emitters in their fluorescent state, one needs to take hundreds of frames to reconstruct one single super-resolved image. Accordingly, this method cannot provide high temporal resolution.

To solve this problem, compressed sensing stochastic optical reconstruction microscopy (CSSTORM) has been developed. [122] Compressed sensing is a signal processing method 
that can separate a signal under conditions of signal sparsity and incoherence. The sparse deconvolution method based on compressed sensing executes a large-scale localization that estimates the position of each emitter. The program localizes every fluorophore in an image simultaneously under the assumption, that signals generated from each fluorophore are independent of each other. The main purpose of the sparse deconvolution is to increase localization accuracy in densely labeled SMLM images. Although sparse deconvolution localizes fluorophores with high accuracy, it cannot deconvolve the images of concentrated emitters. ThreeDeconv and Lucy-Richardson can deconvolve the images of concentrated emitters, but the methods have lower precision than sparse deconvolution. After the development of CSSTORM, the first sparse deconvolution, FAst Localization algorithm based on a CONtinuous-space formulation (FALCON) and SParse Image DEconvolution and Reconstruction (SPIDER) were developed. [57, 77] The new programs enhanced the performance of localization, but even the new programs could not localize concentrated fluorophores in a single frame image.

Martinez et al. developed a new deconvolution program, super-position of virtual point sources (SUPPOSe), to apply the localization method for the analysis of a densely labeled fluorescence image.[73] In this program, all brightness information of an image is expressed as a super-position of single-point emitters having uniform brightness. These emitters are considered as virtual molecules that mimic the properties of the fluorophores, and the image blur can be removed through localization of these virtual fluorophores. One can localize virtual molecules by minimizing the difference between the measured data and the reconstructed image.

This deconvolution method requires a large amount of computation. In a general image processing method, a two-dimensional or three-dimensional array storing brightness information becomes the main variable. However, in order to restore an image, SUPPOSe adjusts positions of virtual molecules, and this process generates additionoal variable about the positions of the virtual emitters. Therefore, we need to handle not only the array of brightness but also the positions of virtual molecules. How to quickly and precisely optimize multiple positions at once is an important factor in determining the performance of this program.

In order to optimize the positions of virtual emitters, SUPPOSe uses a genetic algorithm. In every optimization step of the genetic algorithm, some of the variables about the locations of the emitters are randomly selected, and the selected variables are exchanged 
or transformed. Then, if the new variables are more suitable, the new variables are adopted as the initial values of the variables for the next step. By repeating this process, the variables are optimized to increase the fitness of all variables. Since the genetic algorithm does not need any gradient in the optimization process, it is easy to localize a large number of individual emitters. However, since the locations and emitters to exchange are randomly selected in each optimization process, the same result cannot be expected from the iterative optimization process.[64, 111, 112] This problem can be overcome by using a global optimization algorithm having a verification procedure for the global minimum. The authors of the SUPPOSe paper did also briefly mention the possibility of using a global optimization algorithm, but it has never been used yet.

In this study, I introduce a modified SUPPOSe algorithm for 3D wide-field fluorescence microscopy which I call 3D-SUPPOSe. The missing cone problem of wide-field microscopy can be solved by using SUPPOSe, which can deconvolve three-dimensional images with high precision. In order to maximize the precision of SUPPOSe, the optimization method can be changed to a gradient descent algorithm. By using Adam, a type of gradient descent algorithm that has been recently published and is widely used,[63] the convergence to a global optimum is also increased. The new method achieves consistent and high-precision results, but the method may increase memory consumption due to gradient computation. In addition, since three-dimensional images require additional memory several times more than two-dimensional images, the program is modified to manage memory usage. Through these additional modifications, the SUPPOSe program for three-dimensional wide-field fluorescence microscopy becomes more practical than before.

\subsection{D-SUPPOSe and multi-plane wide-field microscopy for live-cell fluorescence}

By using 3D-SUPPOSe and a wide-field fluorescence microscope, the three-dimensional motions of cells can be rapidly imaged. In particular, a multi-plane microscope that can record signals in three-dimensional space at once can maximize temporal resolution. The high temporal resolution is an advantage of a wide-field microscope and is achieved by recording images without scanning. This technique evolved from imaging in two focal planes published in 2004. Prabhat et al. presented a technique for simultaneously measuring large field-of-view images composed of two lateral image planes.[87] Bi- 
focal imaging has developed into a multi-plane imaging that simultaneously images 9 to 25 focal planes using a grating,[1-3, 3, 4, 83] and a multi-plane microscope that simultaneously images 8 planes using prisms and mirrors.[44, 87] This initial multiplane setup recorded images in 8 focal planes using 3 beam-splitter prisms and 4 mirrors. After that, a precisely designed single prism was used to stabilize the alignment of the prisms and mirrors, and it was successfully used for 3D Super-resolution Optical Fluctuation Imaging (SOFI), and for 3D phase contrast imaging.[32, 33, 44] Multiplane microscopy has a high temporal resolution because it can record a 3D image of a full volume in one shot. However, this technique is also affected by the missing cone problem, which is a disadvantage of wide-field microscopes. In this study, I apply the new deconvolution method, 3D-SUPPOSe, to reconstruct 3D images from data obtained with the multi-plane setup, and I apply this technique for investigating the mechanisms of biological three-dimensional motions.

In order to make this measurement technique more precise, I also introduce a method to measure precisely the parameters required for 3D image rendering. For 3D rendering, images recorded in eight focal planes are vertically aligned at regular intervals and reconstructed into a three-dimensional image. In this process, if the relative brightness of each image plane, and the distancec between image planes are correctly known, one can render an accurate $3 \mathrm{D}$ image. In the papers about the multi-plane setup, these values were calibrated using the three-dimensional brightness and positions of fluorescent beads. However, fluctuations and photobleaching of bead fluorescence may cause errors in each parameter. Therefore, I introduce a method that can correct errors in parameter calibration caused by this experimental error.

The three-dimensional images are arranged according to time, and the arranged images make a movie. The movie composed of several fluorescence images contains information on the movement of fluorescent molecules over time, and by quantifying the movement, the role of molecules in cellullar motion and function can be investigated. Therefore, in order to quantify changes in the spatial distribution of fluorescent molecules, I introduce a new optical flow estimation that can calculate the directions and numbers of moving fluorophores. The introduced calibration, deconvolution, and optical flow estimation will be used in the future to increase the precision of motion detection in live cells imaged with the multi-plane setup, which will broaden the application range of the setup.

In this thesis, I investigate the motion of cardiomyocytes and Dictyostelium discoideum 
cells using deconvolution and the multi-plane setup. Cardiomyocytes are the constituent cells of a heart muscle and which exhibit planar contractile motion. First of all, by measuring the three-dimensional distribution of specific molecules in the cell, the planar motion of the cell and the three-dimensional spatial movement of the molecules are observed. Dictyostelium discoideum is an amoeboid cell with chemotactic behavior that shows directed motion in response to a specific chemoattractant. By examining the changes in the distribution of proteins in these cells during cell migration, the mechanism of cell migration will be investigated. Through the two examples of cardiomyocyte and Dictyostelium discoideum imaging, I will confirm the applicability of the multi-plane setup for three-dimensional imaging of live cells. 



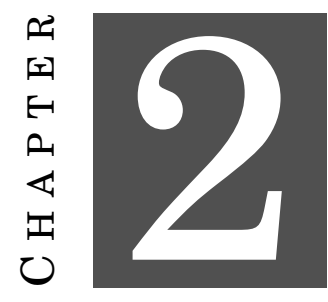

\section{THEORY}

\subsection{Multi-plane Wide-field Fluorescence Microscopy}

\subsubsection{Fluorescence}

Fluorescence occurs after a molecule is electronically excited into an excited state and then relaxes back to the ground state while emitting the excited state energy as a photon. Electronic excitation is a process in which a fluorophore absorbs a photon and switches into an energetically higher electronic state, from which it can relax back to the electronic ground state. This process is explained well using a Jablonski diagram (figure 2.1). The Jablonski diagram shows the vertically arranged electronic, vibrational, and rotational energy states. When a molecule absorbs one photon, the molecule transits from the electronic ground state, $S_{0}$ to the excited state, $S_{1}$. After that, a photon is emitted during the transition from $S_{1}$ to $S_{0}$. This electronic transition is the process of generating a fluorescence photon.

Although fluorescence occurs due to an electronic transition, vibrational-rotational states do also affect the fluorescence spectrum. An electronic state contains several vibrationalrotational states. When a fluorophore absorbs one photon, the molecule transits from the ground vibrational-rotational state of $S_{0}$ to one of the various vibrational-rotational states of $S_{1}$. Conversely, during relaxation, the molecule transits from the ground 
vibrational-rotational of $S_{1}$ to various vibrational-rotational states of $S_{0}$. Therefore, the energies of absorption and emission are not the same as indicated by the vertical arrows in the Jablonski diagram. As shown in figure 2.1, the various distances from the vibrational-rotational ground state of $S_{0}$ to the various vibrational-rotational states of $S_{1}$ are longer than the transitions in the opposite direction. Therefore, the energy generated by relaxation is lower than that of the absorbed photon, and the wavelength of the emitted photon is longer. This is aso called Stokes shift.[47]

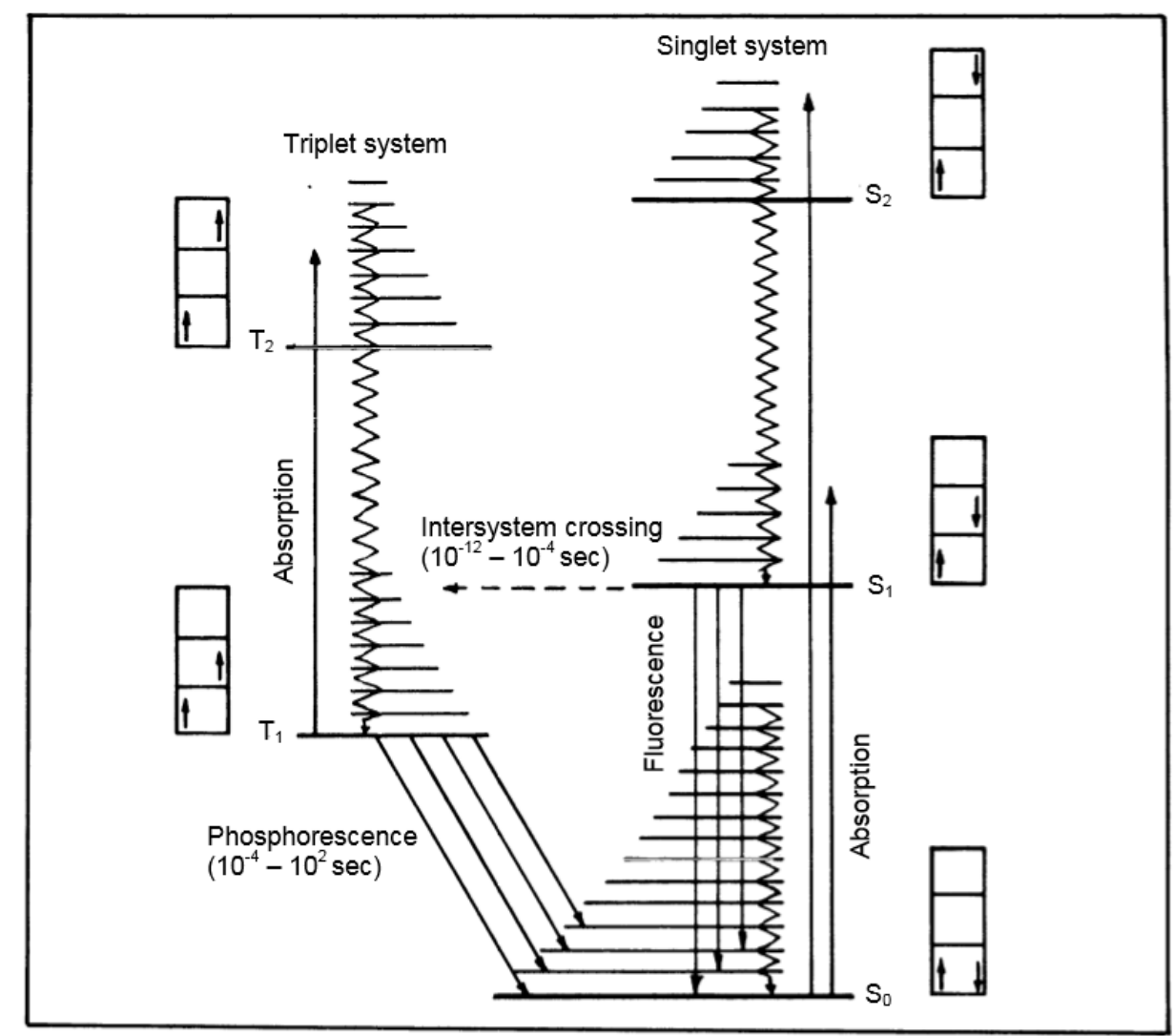

Figure 2.1: The Jablonski diagram of molecular energy states. Fluorescence arises from the electronic transition between the $S_{0}$ and $S_{1}$ states. Phosphorescence occurs due to inter-system crossing between singlet state $\left(S_{1}\right)$ and triplet state $\left(T_{1}\right)$. The vibrational relaxation caused by the overlap between the high energy vibrational energy state and $S_{1}$ is also explained. [117]

Vibrational-rotational states do not only cause Stokes shift, but do also affect the overall shape of the fluorescence absorption and emission spectra. A shape of the spectrum is determined by the distribution of vibrational-rotational states and the possibility of transitions between different states, which greatly depends on the structure of the 
molecule. The difference in the transition possibility between energy states is reflected by intensity differences for each wavelength of a spectrum. Thus, vibrational-rotational states influence the range and shape of the fluorescence spectrum. The absorption and fluorescence emission spectra that contains information about vibrational-rotational states have a symmetrical shape (see figure 2.2).

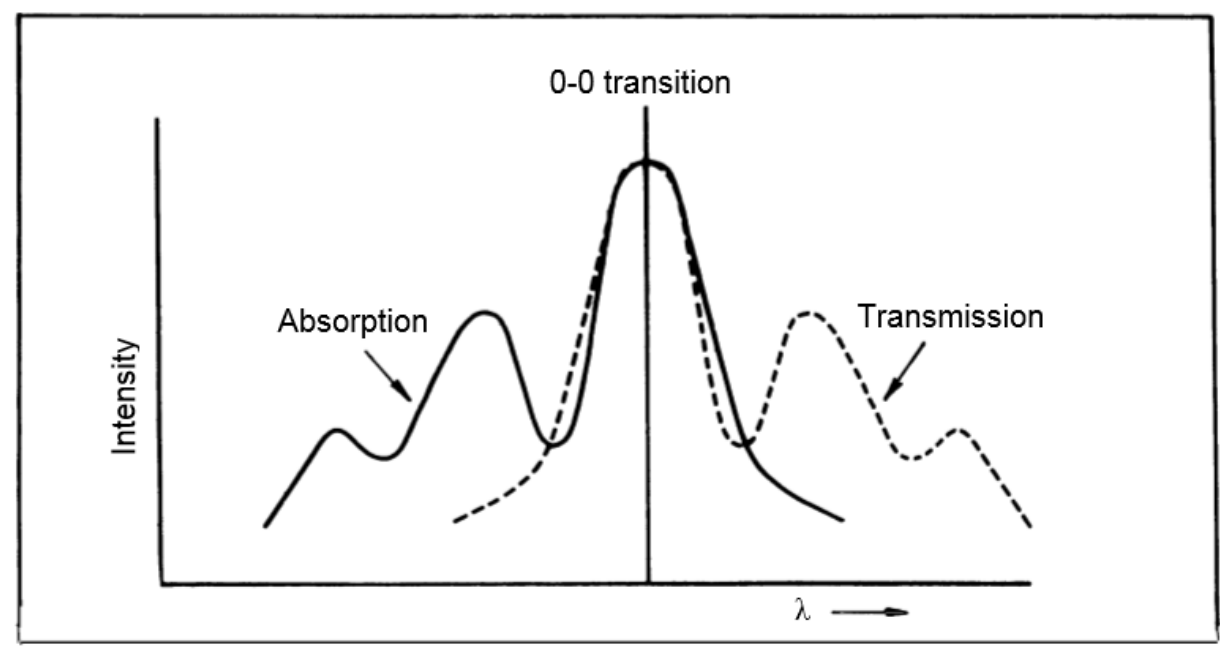

Figure 2.2: Idealized absorption and emission spectra. Absorption and emission spectra have a symmetric shape. The distribution and shape of the symmetric spectrum contains information about vibrational energy states of the molecule. The 0-0 transition is a peak that occurs due to the electronic transition between the ground vibrational-rotational states of $S_{0}$ and $S_{1}$. [117]

The spectral information of each fluorophore is useful for selective signal measurement. Molecules can be selectively excited by illuminating them with light that matches the absorption wavelength of the fluorophore. The shape of a spectrum can be used as a fingerprint of the target fluorophore. Furthermore, changes in the molecular structure or the surrounding environment can induce changes in the spectrum.

\subsubsection{Illuminators and filters to isolate specific wavelengths}

To selectively excite a fluorophore, one uses an appropriate light source. As described in 2.1.1, every fluorophore has its own unique absorption spectrum. By selectively illuminating a sample with light of a specific wavelength, one generates a fluorescence signal of the molecule to be measured. Therefore, selecting a specific wavelength is the first factor to be considered in a fluorescence microscope. For illumination, light sources such as LEDs, lasers, or mercury lamps are used. 
Light sources are classified into monochromatic light sources such as LEDs and lasers and into polychromatic light sources such as mercury lamps. Monochromatic light sources generate light of a single wavelength. They are useful for selective fluorophore excitation. However, the disadvantage is that multiple light sources are required to change the wavelength during measurement. In contrast, a polychromatic light source generates light over a wide wavelength range. To illuminate with a desired wavelength selectively using polychromatic light sources, one employs a monochromater or an optical filter.

The most widely used polychromatic light source in fluorescence microscopy is the mercury lamp. Mercury lamps generate light by electric discharge in vaporized mercury. The high voltage transmitted through the electrodes inside the lamp excites atoms of the mercury gas to an excited electronic state which results in subsequent fluorescence emission. The emission wavelength is determined by the excited electronic energy states that mercury atoms have. In mercury lamps, the emission spectrum covers a wide range from UV to visible light. The spectrum of a mercury lamp is shown in figure 2.3. In microscopy applications, the light of $365 \mathrm{~nm}$ (UV), $405 \mathrm{~nm}$ (Violet), and $436 \mathrm{~nm}$ (Blue) is mainly used.

In order to selectively illuminate with a specific wavelength when using a polychromatic light source, an optical filter or diffraction grating has to be used. An optical filter is a device that selectively transmits light of specific wavelengths. The optical properties of a filter are described by its transmission spectrum, which specifies how the filter modifies the magnitude of each wavelength component. A diffraction grating is an optical component with a periodic structure that diffracts light into multiple beams moving in different directions. The direction of the beam reflected by a diffraction grating depends on the spacing of the grooves of the grating and the wavelength of the light. Thus, a grating acts as a dispersing element. By spatially filtering the reflected light through a slit, one can select the wavelength of the light for illumination. 

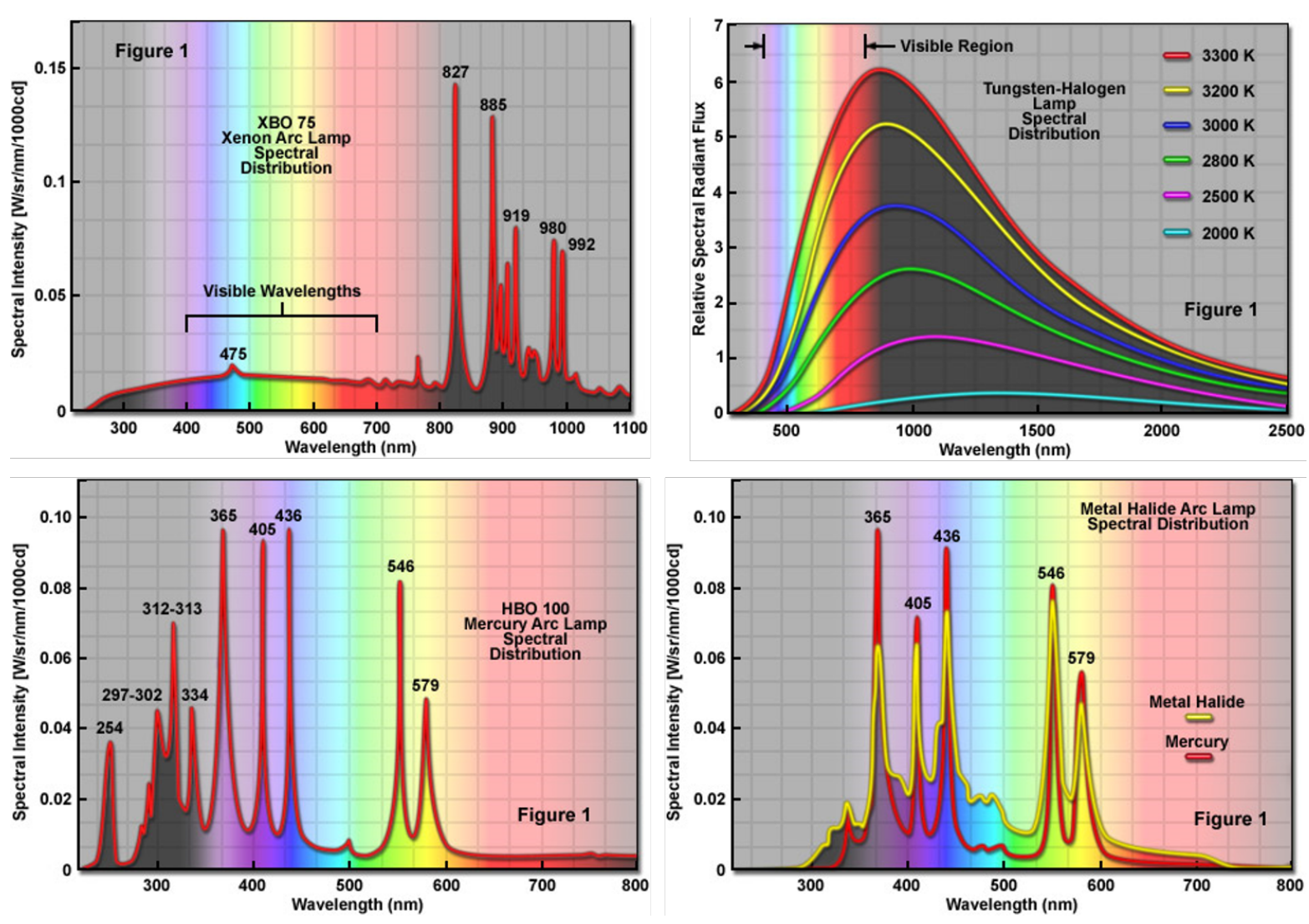

Figure 2.3: Spectra of different types of illumination lamps used in fluorescence spectroscopy and imaging. Left top: xenon lamp, right top: halogen lamp, left bottom: mercury lamp, right bottom: metal halide lamp [28].

A typical monochromatic light source is a laser. The acronym "laser" means "light amplification by stimulated emission of radiation". A He-Ne laser is a good example for understanding the operating principle of lasers. A He-Ne laser has a tube filled with $\mathrm{He}$ and Ne gas in a ratio of 10:1. Electrodes are placed inside the tube, and the electrodes generate a discharge in the gas mixture. The emission light from the discharged gas undergoes multiple reflections by mirrors placed at both ends of the tube, and only a portion of the light is emitted out of the tube. During this stimulated emission process in a laser tube, amplified light having constant phase and polarization is going out from the tube. Thus, we can obtain coherent light with uniform frequency and phase from the laser.

Another characteristic of a laser beam is its collimation. A collimated beam has parallel rays, and the beam has a flat wavefront. Due to coherence and collimation, light with a very narrow wavelength can be used to illuminate a sample without additional filters, 
and focusing into a diffraction-limited spot is also possible. This property is mainly used in microscopy such as confocal laser scanning microscopy (CLSM), where the focal volume is diffraction-limited. The high power density of a laser can generate a strong fluorescence signal, but it also quickly bleaches the fluorescent molecules. Accordingly, we need to control the intensity using attenuation.

A LED (Light-Emitting Diode) is also a light source having a narrow wavelength range like lasers. A LED is a semiconductor light source that emits light when an electric current flows through it. A diode is composed of an n-type semiconductor with free electrons and a p-type semiconductor with holes. When electrons are transferred through the $\mathrm{p}-\mathrm{n}$ junction, which is the surface between the two types of semiconductors, the diode is energetically stabilized by combining the electrons and holes. In this process, a LED emits light within a narrow wavelength range. The color of light is determined by the amount of energy relaxed by electrons passing through the $p-n$ junction of the semiconductor, and the amount of relaxation energy is controlled by the change of the LED's constituent. However, compared to a laser, the emission of a LED has a wider spectral width and is not coherent. However, fast switching is possible through the electric circuit, and its size is generally smaller than the size of lasers.

\subsubsection{Lenses}

The light from illumination sources described above is transmitted to the sample through lenses, and the fluorescence signal from the sample is also collected through a lens system. A lens is a transparent optical element that focuses or disperses light through refraction. Upon refraction, the direction of light is changed due to the difference in light speed between two different media. This phenomenon is called refraction, and refraction follows Snell's law. The lens may form an image by focusing light using refraction. Image formation with a single lens is depicted in 2.4 and follows equation 2.1.

$$
\frac{1}{f}=\frac{1}{a}+\frac{1}{b}
$$

This lens equation describes the relationship between focal length $f$ and object and image distances, $a$ and $b$. 


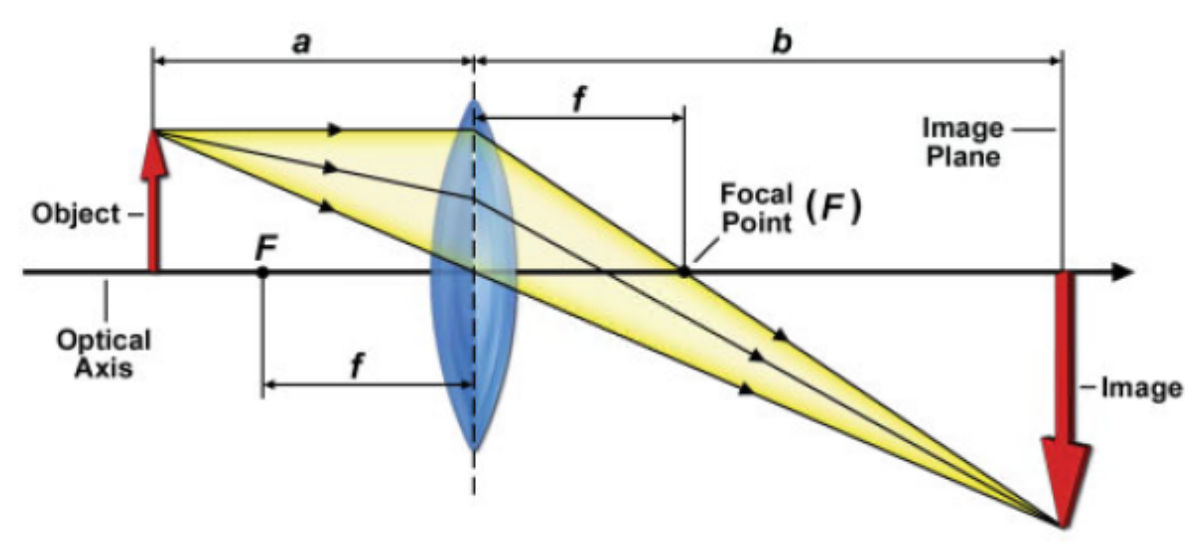

Figure 2.4: Geometrical optics of a thin lens. Focal length $\mathrm{f}$, focus F, object-lens distance a, and lens-image distance $b$ are displayed. [79].

The objective lens of a microscope receives light from the sample and refocuses it into an image. This lens is positioned very close to the sample to achieve a large angle of the cone of light detection. In general, the objective lens of a microscope is composed of several single lenses. The objective lens is characterized by two parameters: magnification and numerical aperture. Magnification is the ratio between the size of an object's image and the size of the object itself. Objective lenses having magnification from $4 \mathrm{x}$ to $100 \mathrm{x}$ are generally used. Along with magnification, an important parameter that characterizes an objective lens is the numerical aperture, which is proportional to the half-angle of the cone of light detection. As this angle increases, an objective lens can collect a larger amount of light from the sample. The numerical aperture is defined as:

$$
\mathrm{NA}=n \sin \theta
$$

where $\theta$ is the half angle of the cone of light accepted by the objective, and $n$ is the refractive index of the sample medium for which an objective is designed. 


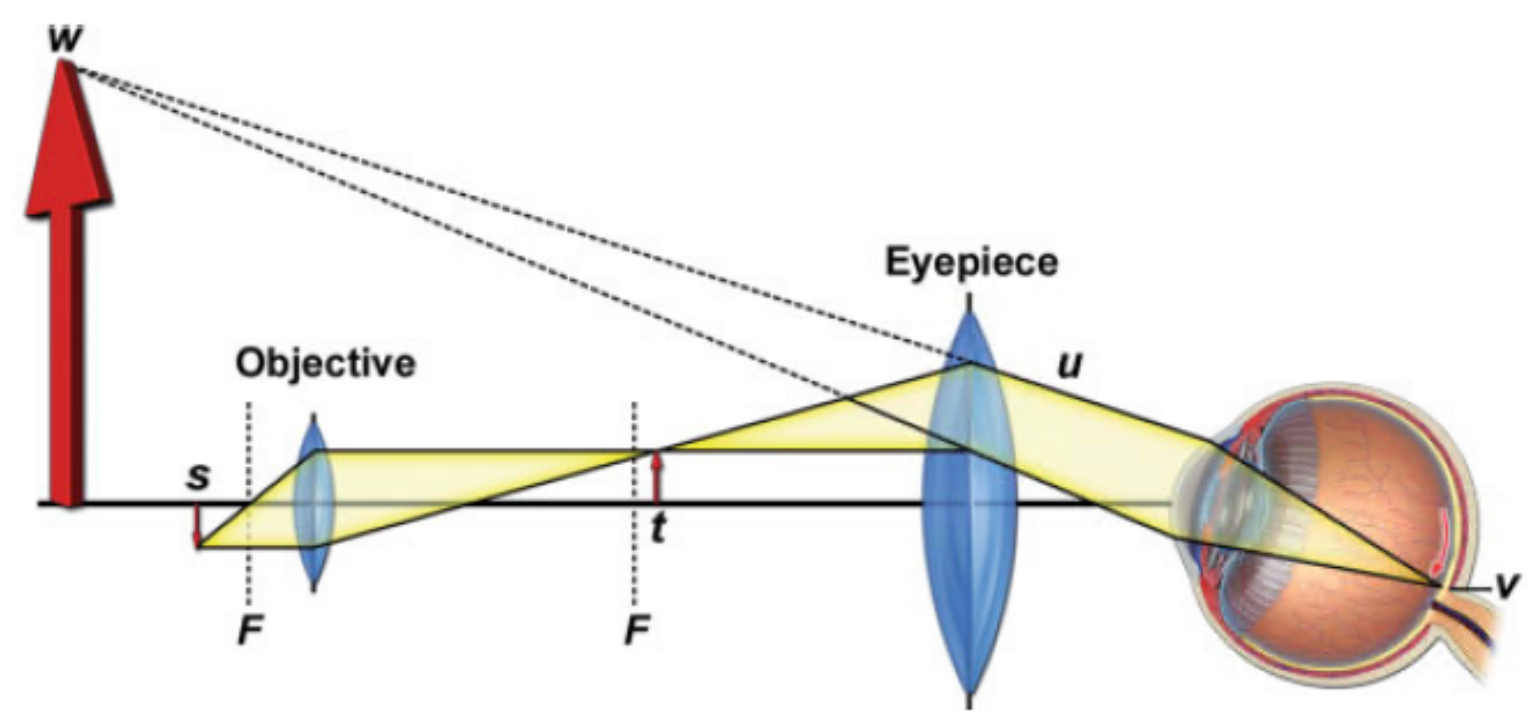

Figure 2.5: The location of real and virtual images in an optical microscope, denoted by $\mathrm{s}$ and $\mathrm{w}$. The specimen in $\mathrm{s}$ is just outside the focal point of the objective lens, resulting in a real magnified image at $t$ of the eyepiece. The primary image of $t$ is located just inside the focal point of the eyepiece, giving rise to a ray emanating from $u$. The cornea and lens of the eye form a real image of the object in the retina in $\mathrm{v}$, which perceives the object as a virtual image magnified in $w$ because of its divergence angle in $u$. [79].

The final image size is determined by the multiplication of the eyepiece and objective magnifications. The image magnified by the objective lens is magnified once again by the eyepiece. In general, since these two lenses have a fixed focal distance, the distance between the two lenses is fixed (see figure 2.5). However, in modern research microscopes, the light collected by an objective lens is collimated into a parallel light beam without focusing, and such a lens is called an infinity-corrected objective lens. In the case of an infinity-corrected objective lens, a separate tube lens is required to refocus the light into an image. Such a configuration has the advantage that the spacing between the tube lens and the objective lens becomes uncritical. Therefore, auxiliary components (such as filters, polarizers, etc.) can be placed between the tube lens and the objective lens with minimal impact on focus.

According to the properties of the objective lens, the beam shape transmitted to the sample is also controlled. Depending on how light is manipulated, microscopes are classified into various types such as confocal and wide-field. One can focus the light into a point on the sample (confocal), or we can illuminate a large sample area evenly (wide-field). 
Confocal microscopes focus light into a point and record the fluorescence brightness from that point. Before intensity detection, the out-of-focus signal is blocked using a pinhole, and this process leads to optical sectioning, which is one of the important differences from wide-field microscopy. Since the confocal microscope is designed to measure the fluorescence intensity from a very small area, it has to scan the sample to obtain an image. In contrast, a wide-field microscope simultaneously illuminates a large area and simultaneously measures the fluorescence from this area. Since this method records images without scanning, wide-field microscopy allows us to measure whole samples in a short time. However, there is no filtering of out-of-focus light as is done in confocal microscopy, so that an image contains contributions from all planes along the optical axis. This will be explained later with the help of the point spread function.

\subsubsection{Point Spread Function (PSF)}

The point spread function (PSF) describes of a microscope is the image of a point emitter. The image of the point emitter is blurred due to the diffraction caused by the wave nature of light. Since the image is blurred by diffraction, the spatial resolution of optical microscopes is limited.

As described above (see 2.1.3), light generated by a point emitter is collected by an objective lens. The minimum distance where two point emitters can still be distinguished in a microscope's image defines the spatial resolution, and it is quantified by Abbe's diffraction limit (1.1). The point-spread function quantitatively describes the threedimensional intensity distribution in image space generated by imaging the light of a point emitter through a microscope. This is mathematically described by a convolution. The convolution integral that computes an image is given by equation 2.4 .

$$
(f \otimes g)(x):=\int_{-\infty}^{\infty} f(\tau) g(x-\tau) d \tau
$$

The convolution formula can be described as a weighted average of the function $f(\tau)$ at position $x$ where the weight function is given by $g(\tau)$ shifted by amount $x$. As $x$ changes, the weighting function emphasizes different parts of the input function.

The result of transferring the PSF from the spatial to the frequency domain is the optical transfer function (OTF) of the microscope: 


$$
\operatorname{PSF}(\rho)=\int \operatorname{OTF}(q) \exp (i q \cdot \rho) d q
$$

Here, $q$ denotes the wave vector, $q=\left(k_{x}, k_{y}, k_{z}\right)$, and $\rho=(x, y, z)$, the position vector. $k_{x}, k_{y}, k_{z}$ are spatial frequencies of wave vectors, and $i q \cdot \rho$ is i times the scalar product of the wave vector $q$ and the position vector $\rho$. The frequency extent of the OTF is related to the spatial resolution of the corresponding optical system, and a large frequency support of the OTF means high spatial resolution.

Depending on the type of microscope, PSF and OTF have different unique shapes and contours (see figure 2.6).
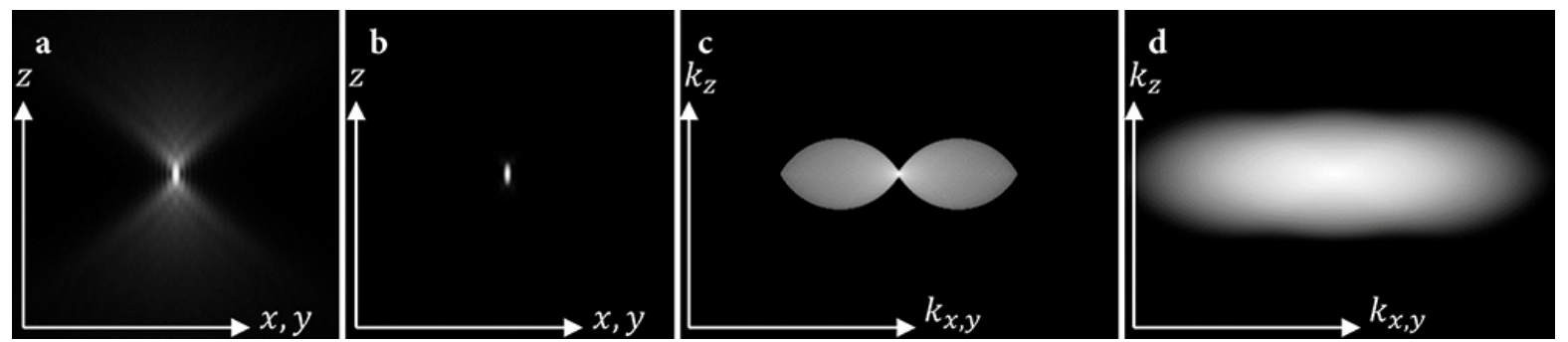

Figure 2.6: a) PSF of a wide-field microscope, b) PSF of a confocal microscope, c) OTF of a wide-field microscope, d) OTF of a confocal microscope, $k_{x}, k_{y}, k_{z}$ are components of wave vector. [102]

\subsubsection{Multi-plane Wide-field Microscopy}

Wide-field microscopy is capable of fast three-dimensional imaging because of it simultaneously illuminates and images the whole field-of-view. However, in order to obtain a three-dimensional image with a two-dimensional sensor, several two-dimensional images have to be recorded. In order to simultaneously acquire fluorescence signals generated in three-dimensional space using a wide-field microscope, additional optical components that separate images for several focal planes are required. Here, I introduce a multi-plane setup that allows simultaneous recording of fluorescence images in a three-dimensional space.

The multi-plane setup, the main microscopy technique used in this thesis, uses beam splitters and mirrors to simultaneously image the fluorescence of a three-dimensional volume. The theoretical background of multi-focal imaging was described by Prabhat et al. in 2004. They introduced bi-focal imaging. [87] They successfully imaged two different 
planes simultaneously using the optical property that the detector is slightly shifted along the optical axis, the conjugated plane in sample space shifts accordingly, as shown in figure 2.7. Toprak et al. also imaged and analyzed 3D particle trajectories in the same way in 2007. [108] Both methods have been implemented to collect multiple images from different focal planes by dividing the signal into two beams using a beam splitter.

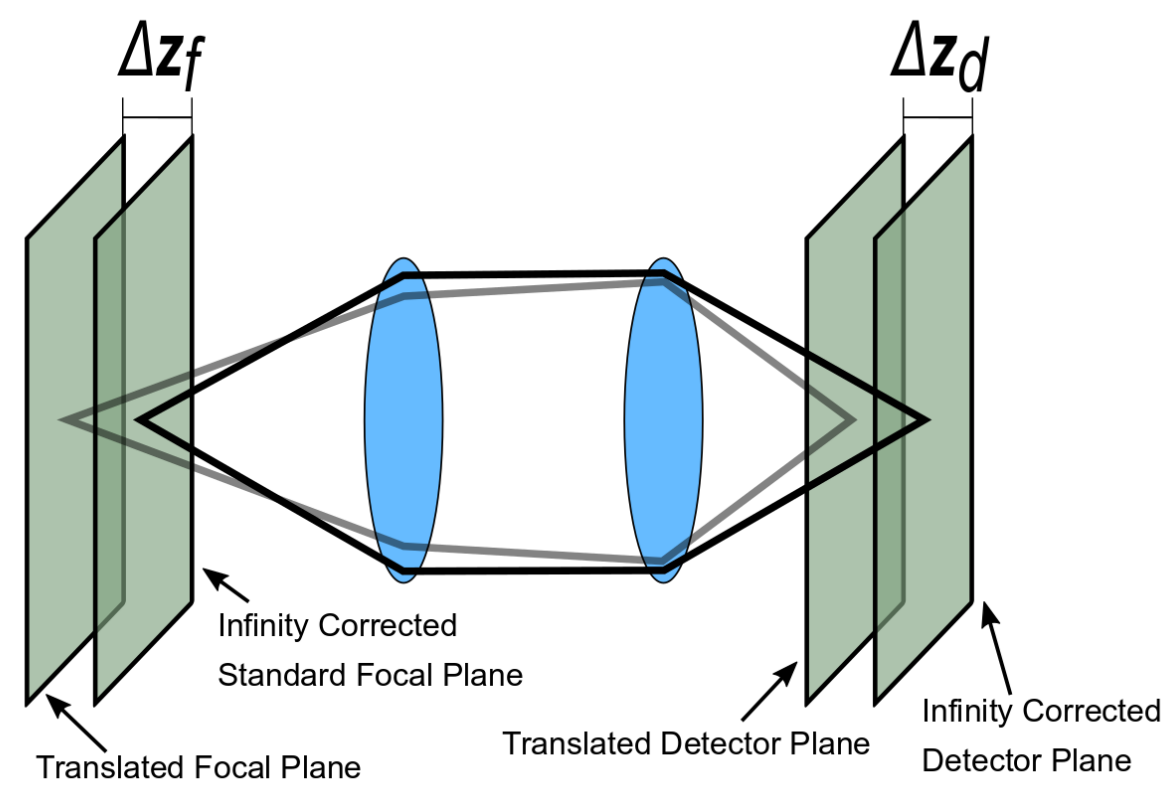

Figure 2.7: The basic concept of a bi-focal image. The detector plane moves a certain distance according to the movement of the focal plane. Using this principle, simultaneous imaging of two image planes is possible. [87]

Bi-focal imaging has been extended to multiplane imaging using multiple beam splitters and mirrors. [60, 113] In 2014, Geissbuehler et al. introduced a method for simultaneously recording eight images, and they used the technique for cell imaging with super-resolution microscopy. [44] Three beam splitters and four mirrors were used to simultaneously acquire images from multiple focal planes as shown in figure 2.8 . The inter-plane distance, $\delta z$, is set by the axial magnification of the objective lens, $M_{a}$, and the distances between the mirrors, $d$, as indicated in figure 2.8. The relation between the parameters is given by following equation:

$$
\delta z=\frac{d}{M_{a}}
$$


The well-aligned mirrors and beam-splitters split the collected fluorescence light into eight images corresponding to eight focal planes. Two cameras acquire images with different optical path lengths by shifting one camera by a distance $4 d$.

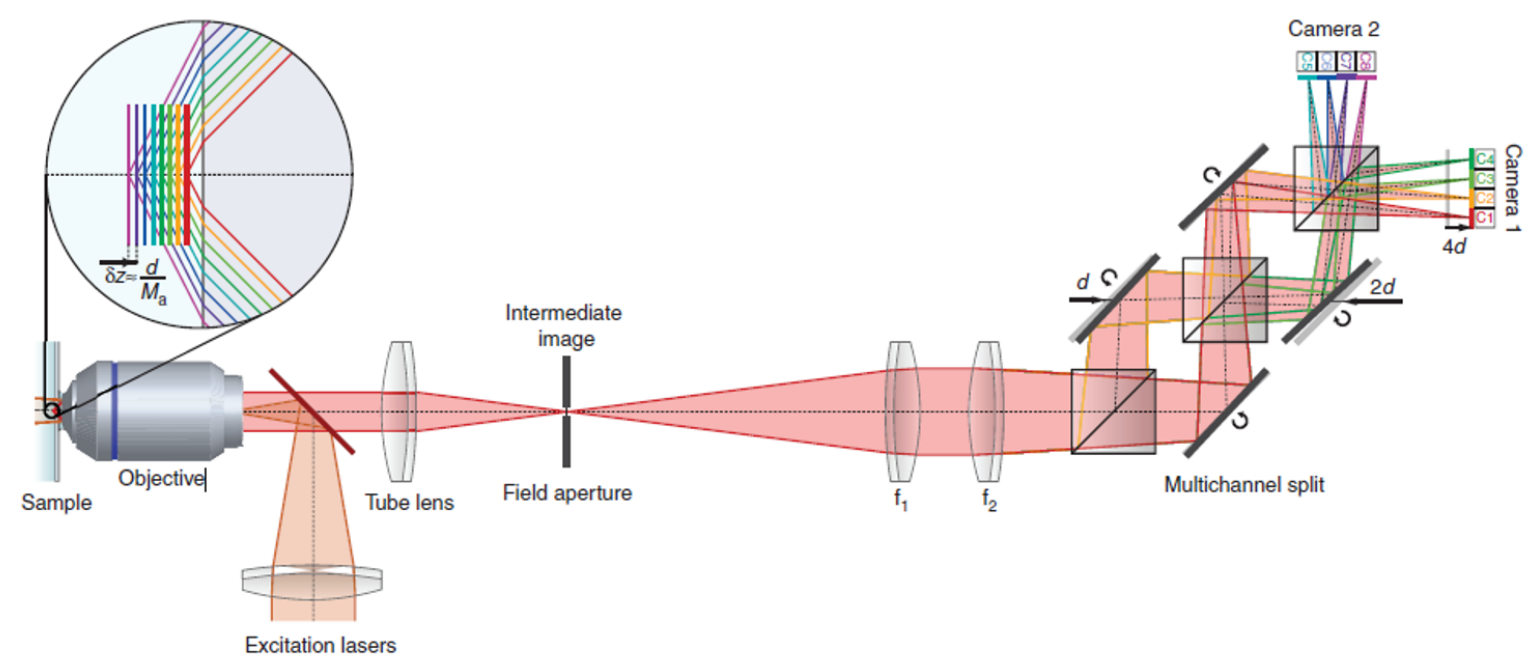

Figure 2.8: Multi-plane wide-field fluorescence microscope. The excitation laser is focused into the back aperture of the objective to create a large illumination field. Sample features in several focal planes are imaged simultaneously by two cameras. [44]

The configuration of this optics was implemented as a single beam splitting prism, and the prism-based method was used for phase-contrast microscopy.[32] A prism-based multi-plane technique was used with structured illumination microscopy as well as phase-contrast microscopy. [32, 33]

As shown in figure 2.9, the prism-based multi-plane setup records fluorescence from a three-dimensional volume as eight two-dimensional images generated by the image splitting prism. [32] In the multi-plane microscope, the sample is illuminated in the entire field-of-view (FOV) in the same way as is done in a general wide field microscope, and the fluorescence from the eight focal planes in the FOV is divided by the prism and detected by the two cameras. Like the previous design of the multi-plane setup, the distance between each focal plane depends on the magnification of the objective lens and the distance between the reflecting surfaces of the prism. However, since the fluorescence light travels through the prism, the refractive index of the prism material, $n$, also affects the inter-plane distance. Therefore, the equation of the inter-plane distance is changed to 


$$
\delta z=\frac{d}{n M_{a}}
$$

The distance between the reflecting surfaces is fixed by the design of the prism and is adjusted in a way that satisfies the Nyquist-sampling condition. Therefore, when the prism is used in multi-plane setup, there is no additional variable that can control the distance between the focal planes except the magnification of the objective lens.

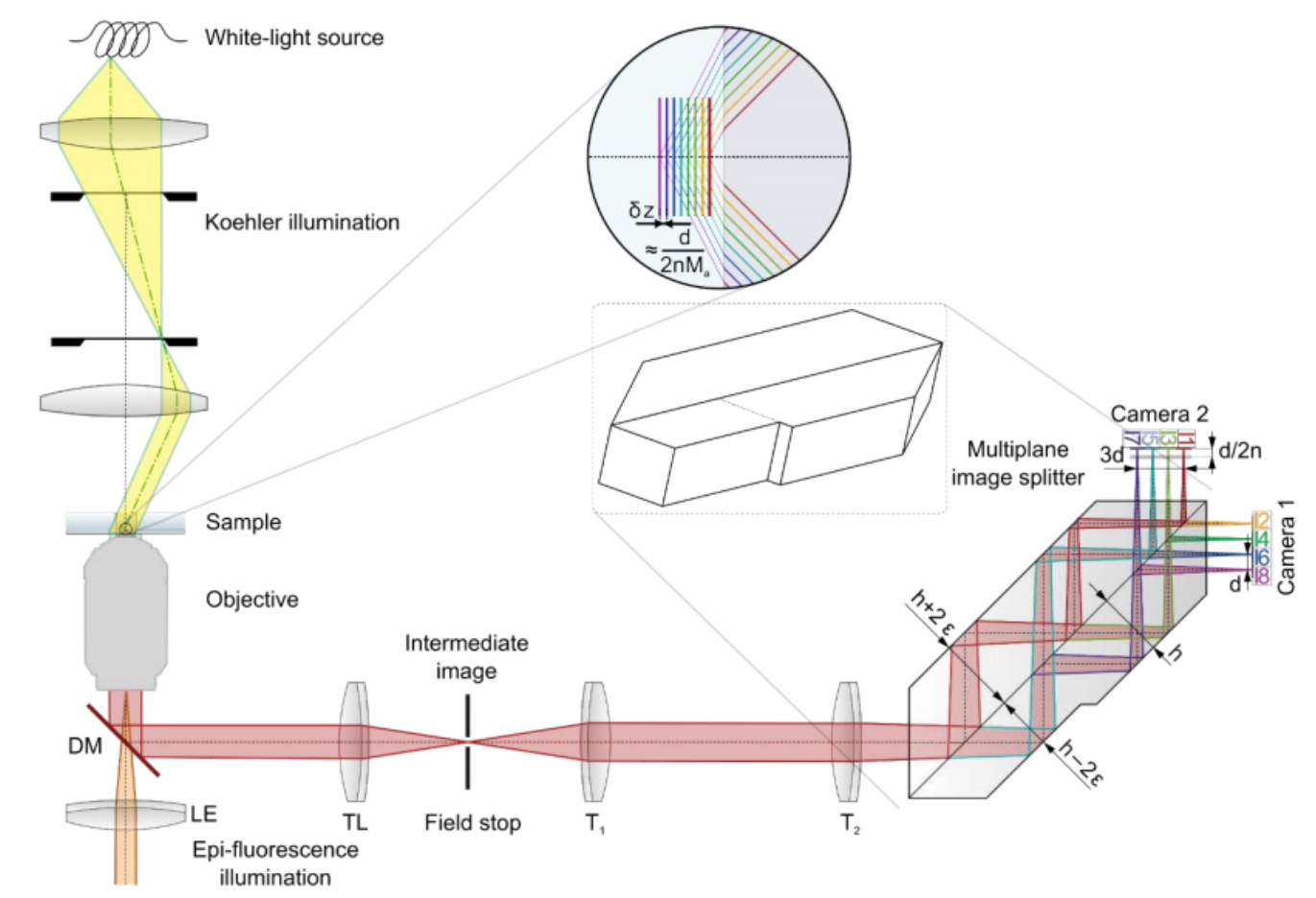

Figure 2.9: Setup of the multi-plane wide-field microscope. For wide-field epi-illumination phase imaging, the setup was combined with Koehler trans-illumination. The multiplane image segmentation prism splits light into eight image planes evenly spaced along the optical axis. DM dichroic mirror, LE lense, TL tube lense, T1 and T2 lenses. [32]

\subsection{Digital Image Processing}

The image generated by a microscope is recorded with an imaging sensors and stored as a digital signal. In this process, the image is blurred by the diffraction, and additionally affected by various types of noise. In this section, the process of image blurring by diffraction and corruption by noise will be summarized in mathematical formulas. 


\subsubsection{Image sensor and sampling}

The image generated by a microscope is usually recorded with an imaging sensor and the stored as digital information on a computer. An imaging sensor is a device that converts light intensity (number of photons per area per time) into an electrical signal. Typically used sensors are charge-coupled device (CCD) and complementary metal-oxidesemiconductor (CMOS), and they are classified according to the difference how they record and amplify the signal. Both types of detector consist of individual sensor elements (pixels) that generate an electrical signal proportionally to the impinging intensity of light.

(a)

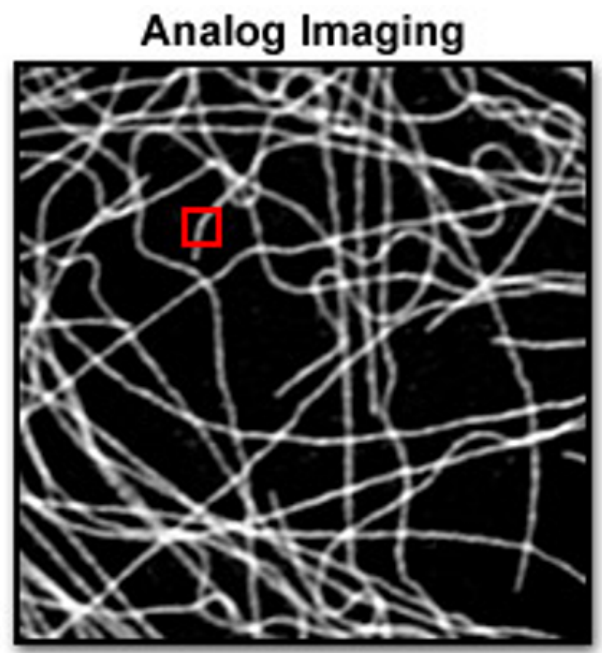

(b)

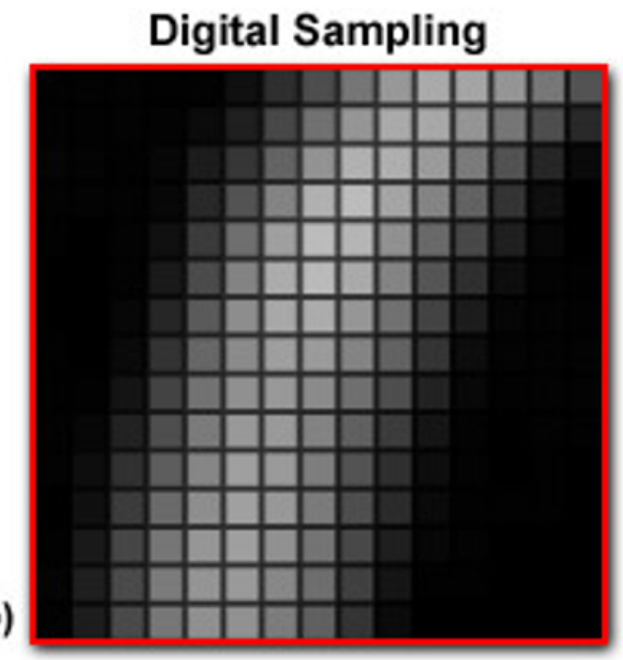

\section{Analog and Digital Signals}

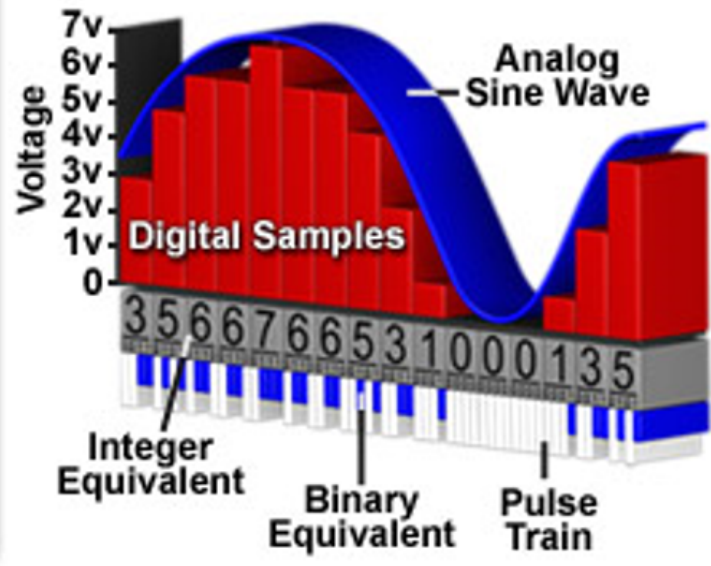

\section{Pixel Quantization}

\begin{tabular}{|c|c|c|c|c|c|c|c|c|c|c|c|c|c|c|}
\hline & 3 & & 2 & & N & 47 & 78 & & & & & 149 & & \\
\hline 3 & 2 & 3 & 3 & 11 & 31 & 72 & 113 & 150 & 170 & $1 \in 3$ & 148 & 148 & 78 & 41 \\
\hline 5 & 3 & 2 & 7 & 28 & 51 & 100 & 147 & 177 & 175 & 155 & 12.] & 32 & 38 & 10 \\
\hline$\frac{5}{3}$ & 3 & 3 & 8 & 41 & 83 & 132 & 177 & 138 & 165 & 132 & 98 & 62 & 15 & $\bullet$ \\
\hline 2 & 0 & 3 & 16 & 59 & 111 & 160 & 188] & 181 & 145 & 105 & 72 & 31 & 7 & 0 \\
\hline 0 & 0 & 3 & 28 & 75 & 131 & 172 & 186 & 170 & 132 & 35 & 47 & 13 & 3 & 2 \\
\hline 0 & 0 & 7 & 38 & 90 & 411 & 168 & 172 & 160 & 113 & 67 & 28 & 5 & 3 & 2 \\
\hline 2 & $\overline{0}$ & 10 & 51 & 6003 & 139 & 159 & 155 & 132 & 5 & \begin{tabular}{|l|}
52 \\
\end{tabular} & 13 & 3 & 3 & 3 \\
\hline 2 & 2 & 20 & 67 & 114 & 944 & 152 & 139 & 111 & 78 & 34 & 8 & 2 & 3 & 3 \\
\hline 3 & 7 & 34 & 78 & 123 & 552 & 55 & 131 & 36 & ๓) & \begin{tabular}{|l|}
21 \\
\end{tabular} & 3 & 0 & 3 & 2 \\
\hline 3 & 13. & 49 & 93 & 134 & 859 & 654 & 126 & 83 & 47 & \begin{tabular}{|l|}
13 \\
\end{tabular} & 3 & 0 & 2 & 0 \\
\hline 0 & 46 & $60 \mathrm{P}$ & 106 & 42 & 160 & 152 & 123 & 78 & 43 & 10 & 2 & 2 & 2 & 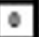 \\
\hline$\overline{2}$ & 25 & 70 & 198 & 150 & 859 & 145 & 106 & 72 & 31 & 7 & 3 & 0 & 0 & 0 \\
\hline 5 & 31 & 74 & 123 & 49 & 854 & 136 & 110 & 64 & 21 & 2 & 2 & 0 & 0 & 0 \\
\hline & 29 & 74 & & $144 \mathrm{E}$ & & 124 & & 52 & 11 & 0 & & 0 & 0 & \\
\hline
\end{tabular}

Figure 2.10: How a digital image is recorded, (a) Digital sampling of an analog image, (b) Resulting image from digital sampling, (c) Matrix form of the digital image [29]. 
Due to its structural characteristics, the image recorded by through the sensor has a unit element called a pixel which constitutes the minimum area of a digital image. The corresponding unit of a digital $3 \mathrm{D}$ volumetric representation is called voxel. An image with continuous information is converted into a set of discrete numbers by a process called sampling (see figure 2.10).

The size of a pixel is related to the number of samples in a given space. The number of samples read per unit area or volume is called the sampling rate or spatial frequency of the image. The frequency domain of an image shows the range of spatial frequencies that the image can contain. This similar to the relation between spatial resolution of the optical system and the extent of the frequency support of the OTF (see 2.1.4). In order to preserve the full information of the image, it must be sampled with a frequency which is larger than twice the maximal spatial frequency contained in the image. This follows from the Nyquist-Shannon sampling theorem, and sampling under these conditions is called super-Nyquist sampling. [23, 69] Conversely, if this condition is not met, the sampling distorts the spectrum of spatial frequencies of the image, and this phenomenon is called aliasing. [84]

\subsubsection{Convolution and Image Kernel}

Image distortion may occur due to the influence of various measurement conditions, such as the characteristics of the lens and noise. The distortion can be expressed using convolution integral in equation 2.4. In the equation, the applied function transforming an image is called a kernel. 
Original Image Data

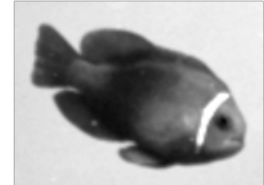

Box Blur Kernel

$$
\frac{1}{9}\left[\begin{array}{lll}
1 & 1 & 1 \\
1 & 1 & 1 \\
1 & 1 & 1
\end{array}\right]
$$

Edge Detection 2

$$
\left[\begin{array}{ccc}
0 & 1 & 0 \\
1 & -4 & 1 \\
0 & 1 & 0
\end{array}\right]
$$

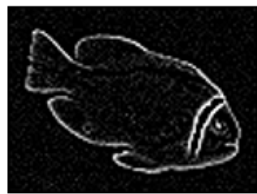

Gaussian Blur (3×3)

$$
\frac{1}{16}\left[\begin{array}{lll}
1 & 2 & 1 \\
2 & 4 & 2 \\
1 & 2 & 1
\end{array}\right]
$$

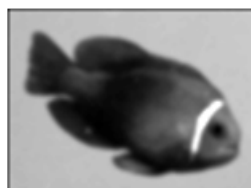

\section{Sharpen}

$$
\left[\begin{array}{rrr}
0 & -1 & 0 \\
-1 & 5 & -1 \\
0 & -1 & 0
\end{array}\right]
$$

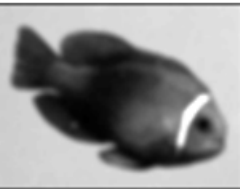

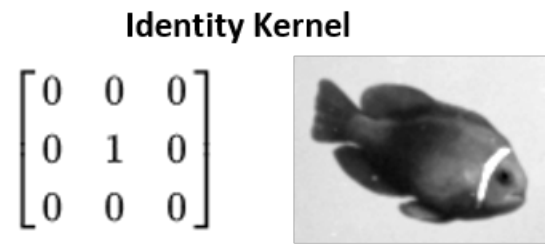

Edge Detection 1

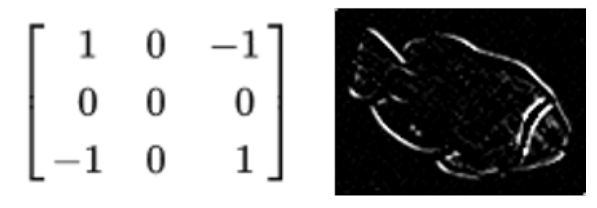

Edge Detection 3

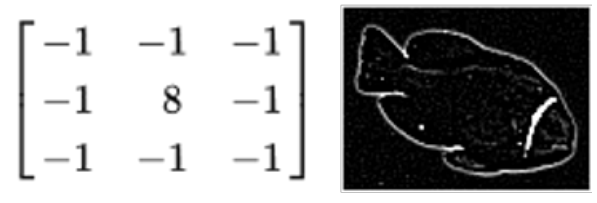

Gaussian Blur (5x5)

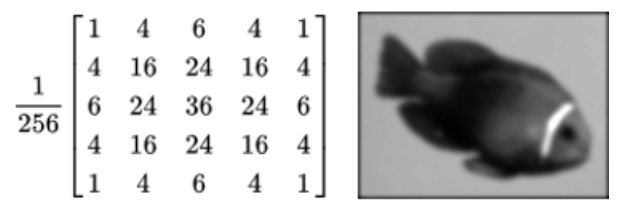

Unsharp masking

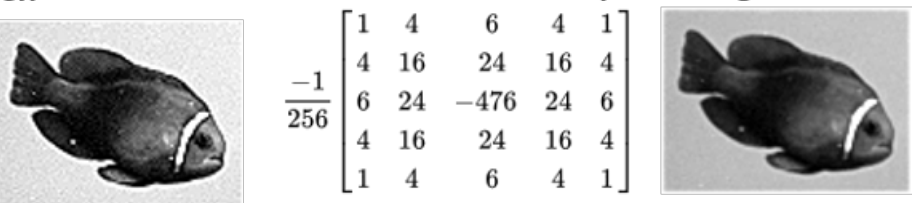

Figure 2.11: Examples of image kernels

If the PSF is used as an image kernel, the convolution integral expresses the imaging process of the fluorescence signal by the microscope. Conversely, we can use this mathematical expression to approximate the position of the point emitter from the blurred image. The following equation 2.7 describes the relationship between the fluorescence signal distribution $(x)$, the image obtained by a microscope $(y)$, and the PSF $(H)$.

$$
y=H \otimes x
$$




\subsubsection{Noise}

We can express an image by the convolution described above, but we need additional method to express undesired signal mixing. The signal that we do not want to measure but that is included in the image is called noise. There are noise contributions that can be blocked in an experiment like an external light source, but there are noise contributions that cannot be eliminated like Poissonian noise and thermal noise.

Poissonian noise, or shot noise, is generated by the quantum behavior of light. The standard deviation of this distribution is proportional to the square root of the mean.[120] Therefore, the signal-to-noise ratio (SNR) is given by

$$
S N R=\frac{N}{\sqrt{N}}=\sqrt{N}
$$

According to this equation, the SNR increases in proportion to the square root of $\mathrm{N}$, which is the number photons collected by a camera pixel.

Thermal noiseis another noise source that can affect an image. It was discovered by John Bertrand Johnson in 1926, and it is caused by the motion of free electrons in a conductor. As the temperature increases, the motion of free electrons is becoming more energetic. It is also called Gaussian noise because the noise follows a distribution of a Gaussian random variable. Thermal noise is independent of the number of photons.

The effect of these two sources of noise on the image can be expressed as follows:

$$
Y=\mathbf{P}(H \otimes X)+\mathbf{N}
$$

In this equation, $\mathbf{P}$ represents a Poissonian random variable with an expectation value given in the brackets. $\mathbf{N}$ is additive noise modeled as a Gaussian random variable. [17] [74] Since this noise is independent from the signal level, it is expressed in the additive form to the product of the image and Poissonian noise. 
Equation 2.9 is visualized in figure 2.12. As shown in the first panel, the three-dimensional image $Y$ of a point emitter is defined by the PSF, $H$. The convolution between the PSF and the point emitter is expressed in the first line, and the picture on the right side is the blurred point emitter image $H \otimes X$. The convolution result does not include the effect of noise. Here I consider only additive noise $N$, which is Gaussian noise, in order to express the process concisely. After adding noise the image of the point emitter is shown in the bottom-right panel of the figure.
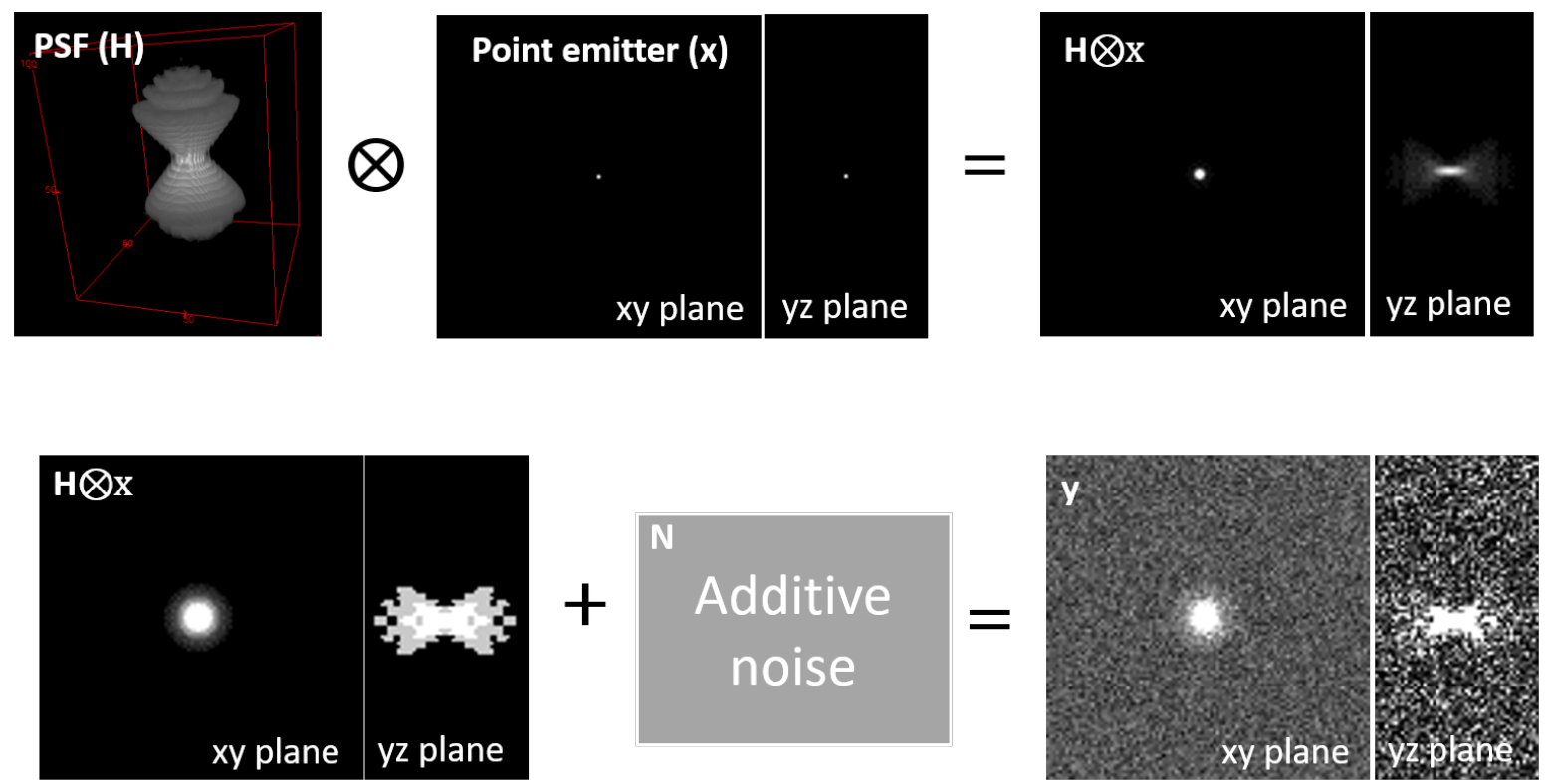

Figure 2.12: The PSF and noise effect on image. We can use convolution to describe the effect of PSF on image. If the additive noise is included, then we obtain the noisy image at bottom-right..

\subsubsection{Deconvolution as an Ill-posed Inverse Problem}

Above, I explained the blurring and noise in imaging. Now, I will explain how to reduce these effects using equation 2.9 .

The recorded image is $Y$. Ideally, to remove both the effects of noise ( $P$ and $N)$ and PSF $(H)$, we should solve the equation 2.9 to find the most likely values of $X$. The problem of finding $\mathrm{X}$ by reversing the convolution integral is called an inverse problem. For the above equation, it is difficult to find a unique solution for $X$ because there is generally less information in $\mathrm{Y}$ than in $\mathrm{X}$. If a problem has no single unique solution, we call it an ill-posed inverse problem. [106] 
In the case of image processing, an image close to $X$ can be estimated from the equation 2.9 by implementing certain constraints and assumptions into the inverse problem. This process is called image deconvolution. Since the image deconvolution process is an ill-posed inverse problem, it is difficult to obtain an exact solution of $X$. However, many algorithms have been developed to efficiently approximate the solution of this problem, and we can approximate the image $X$ using the algorithms. [94, 99] The first deconvolution algorithm developed for inverse problems was the Wiener filter, and this algorithm was developed to reconstruct a radio signal rather than image deconvolution. After that, new deconvolution programs were developed using efficient approximation methods of the solution, $X$, or suggesting a new model in order to efficiently remove noise. Based on the mathematical method that will be introduced in the next chapter, various image deconvolution algorithms have been designed.

\subsection{Mathematical Tools to Solve the Inverse Problem}

In this section, I explain mathematical techniques for deconvolution as a method for sharpening an image.

\subsubsection{Regression Analysis}

First, I will explain regression analysis, which is often used to solve an inverse problem. Regression analysis is a mathematical technique that discovers the relationship between two or more variables in a specific model function. Regression analysis is used to analyze the trend of measurement data. After setting a model function of several parameters, the parameters are optimized by minimizing the difference between the model function and measurement data. To compare the measured data and the model function, we compute the absolute or square value of the difference between the two. By adjusting the parameters so that the calculated values are minimized, we can find the optimized model function that describes best the measured data. For this process, we use a simple linear regression, and various types of functions such as logarithmic and exponential functions can be utilized to make complex model functions.[86, 107]

The first approach of this regression analysis was the least-squares method introduced by Adrien-Marie Legendre and Carl Friedrich Gauss. Sir Francis Galton started to use the name of regression in his work.[11, 43, 65] He studied the effect of heredity on people's height. He found that the offspring of tall people tend to be taller than average, but their 
heights converge to the overall people's average height.[42] The term of regression was used to describe it as a tendency to regress to the mean value. The basic equation of regression analysis is the following equation:

$$
y=h\left(x_{1}, x_{2}, x_{2}, \cdots, x_{n} ; \beta_{1}, \beta_{2}, \beta_{3}, \cdots, \beta_{n}\right)+\epsilon
$$

A general regression analysis formula consists of dependent variables $\left(x_{n}\right)$, independent variables $(y)$, unknown parameters $\left(\beta_{n}\right)$, and error term $(\epsilon)$. The value of error term means the difference between the dependent variable and model function composed of independent variables and unknown parameters. By minimizing this error value, we can optimize the model function $(h)$. When the model function reproduces only a part of the data, it is called underfitting. Contrary, when a function is too closely following every data point, it could be a sign of overfitting. Figure 2.13 shows an overfitting example. The model function leads to a lower error than a simple model, but the prediction can be wrong.
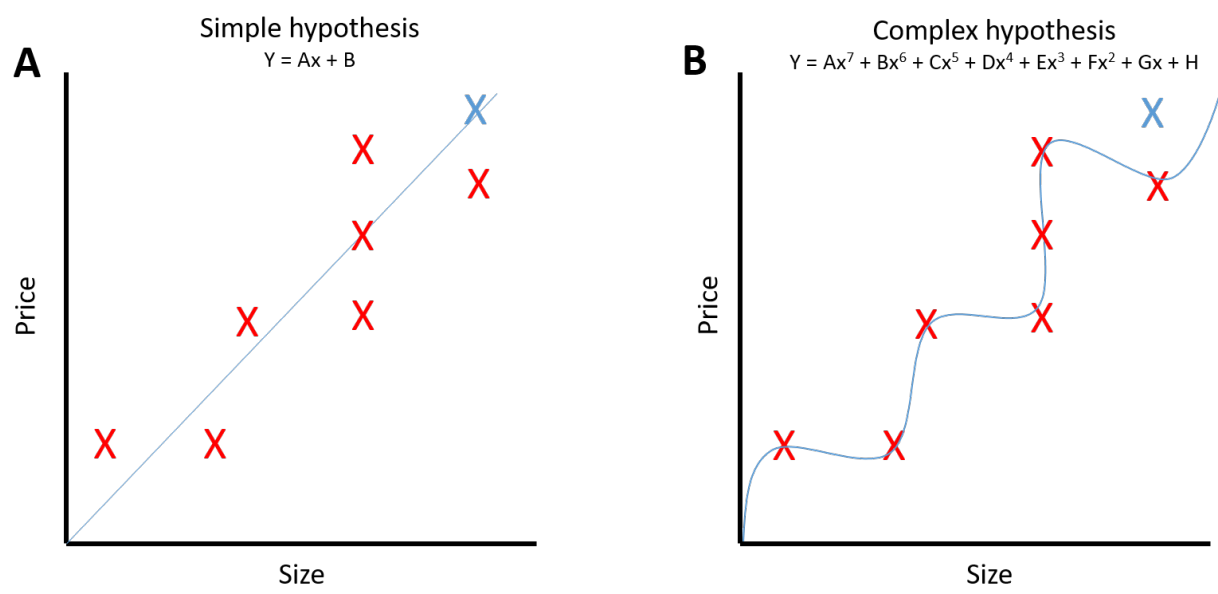

Figure 2.13: Overfitting example

\subsubsection{Penalized Regression}

The overfitting shown in figure 2.13 lowers the accuracy of data predictions. To avoid overfitting and ignore the noise in data, we add an error term to the model function. This error term mainly consists of specific parameters included in the model function, 
and we can keep the parameters in a certain range. The error term used here is called the penalty parameter, and this method is called penalized regression. The penalized regression result depends on the type of penalty function. In many cases, the square (Ridge regression) or absolute value (Lasso regression) is used as a penalty functions.

In the absence of noise, $X$ can be estimated from the measured image $Y$ by calculating a value that minimizes the difference between $H \otimes X$ and $Y$. The two penalized regressions are expressed as the following equations:

$$
\Phi(X)=|Y-\mathbf{H} X|^{2}+\lambda \Sigma|X|
$$

$$
\Phi(X)=|Y-\mathbf{H} X|^{2}+\lambda \Sigma X^{2}
$$

In order to optimize the parameters in $X, \Phi(X)$ is minimized, and $\Phi(X)$ is called an objective function or cost function. In order to prevent overfitting, we can use $X$ as a penalty parameter. Then, the difference between the model function and $Y$ is minimized, keeping the value of $X$ as low values. In Lasso Regression, the absolute value of $X$ is used in the objective function (see equation 2.11), and in Ridge Regression, the square of $X$ is used as a penalty parameter(see equation 2.12). These two methods have very similar structures, but the results have different characteristics. As shown in figure 2.14, if the square value of two variables is set to stay in a certain range, both variables are adjusted to find an appropriate value. However, when the sum of the absolute values of two variables is set to stay in a certain range, a variable that has relatively little influence on the objective function converges to 0 . This phenomenon occurs when the power of the penalty parameter is less than or equal to 1 , and this case is called sparse regression. This method increases the sparsity of the parameters. Therefore, it is used in various ways, such as analyzing highly sparse data or reducing the amount of parameters used in the optimization process. This method is used in the recent image deconvolution methods, which will be discussed in the next chapter. 

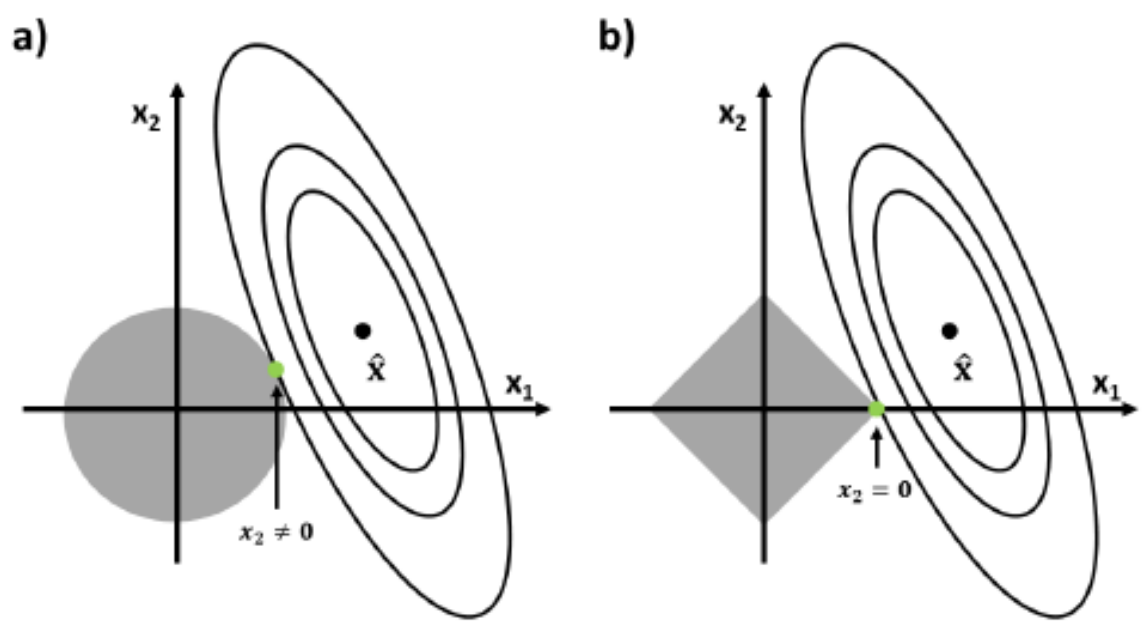

Figure 2.14: Representation of the (a) Ridge regression (constraint region: $x_{1}^{2}+x_{2}^{2} \leq S$ ), and (b) Lasso regression (constraint region: $\left|x_{1}\right|+\left|x_{2}\right| \leq S$ ) estimation. The black ellipses are the contours of the least squares error function, while the gray areas are the constraint regions. The green dots indicate where the penalized solution is tangent to the constraint region. [109] [85]

\subsubsection{Optimization Method}

Regression analysis is applied widely due to the development of artificial intelligence (AI) technology. Since general AI techniques deal with a huge amount of data, it is necessary to quickly identify the trend of the data and transmit the result to the next calculation step. In this process, regression analysis is used, and this method has been developed more and more according to the need. For efficient regression, minimization of the objective function to optimize the parameters quickly are also being studied.

As described above, the first step in regression analysis is to define an objective function. By minimizing the objective function, we can optimize the parameter that corresponds to a specific condition. The process of finding parameters for the conditions is called optimization. Optimization methods have been developed in various ways since about 200 years ago.[98] The methods have been developed in various forms, such as using statistics, calculating the slope of the objective function, and using a genetic concept of biological systems. In particular, gradient descent algorithms and genetic methods are widely used in recent AI-related research. In this thesis, I will focus on gradient descent, and I will discuss the use of genetic methods. Therefore, these two methods are introduced in this section. 
Augustin-Louis Cauchy first proposed the gradient descent algorithm in 1847, and Haskell Curry in 1944 presented the logical basis for the gradient descent algorithm by proving the convergence properties of nonlinear functions. [26, 66] This optimization algorithm updates target information gradually. Stepping in the direction of the gradient will lead to a local maximum or minimum of a function. The main iteration of the algorithm is as follows:

$$
x_{n+1}=x_{n}-\gamma \cdot \nabla F\left(x_{n}\right)
$$

When the value of the function, $F(x)$ is defined and differentiable in a neighborhood of a point $x_{n}$, we subtract the gradient value of the objective function from $x_{n}$. To adjust the effect of gradient size to $x_{n+1}$, we add a learning rate, $\gamma$, in the equation. If the learning rate is too big, the function is greatly affected by the gradient. Accordingly, the moving speed to the minimum becomes fast, but the position tends to oscillate around the minimum. Conversely, a small learning rate can slow down the convergence towards the minimum.

Gradient descent algorithms also tend to be trapped in local minima, so to overcome this limitation, a new algorithm that adds a momentum parameter, Adam (Adaptive Momentum Estimation), was introduced in 2014 by Kingma et al.[63] The idea of a momentum parameter was introduced for the first time by Rumelhart et al. in 1986.[92] After that, by using the momentum parameter together with a learning rate in the RMSprop algorithm,[80] Kingma et al. developed Adam, one of the gradient descent algorithms that currently has a wide range of applications in computational research. How to update a parameter in Adam is as follows: [63]

$$
\begin{aligned}
& m_{n}=\beta_{1} \cdot m_{n-1}+\left(1-\beta_{1}\right) \cdot \nabla F\left(x_{n-1}\right) \\
& v_{n}=\beta_{2} \cdot v_{n-1}+\left(1-\beta_{2}\right) \cdot \nabla F\left(x_{n-1}\right)^{2} \\
& \hat{m}_{n}=\frac{m_{n}}{1-\beta_{1}^{n}} \\
& \hat{v}_{n}=\frac{v_{n}}{1-\beta_{2}^{n}} \\
& x_{n}=x_{n-1}-\alpha \cdot \frac{\hat{m}_{n}}{\sqrt{\hat{v}_{n}}+\epsilon}
\end{aligned}
$$


In Adam, it is essential to update the gradient information to $x_{n}$.(2.18) In the process of updating the gradient information to parameters, we use two equations. One equation is defining the two momentum operators (first order momentum, $m$ (2.14), and second order momentum, $v$ (2.15), and the next equation is converting the momentum operators to a bias-corrected form ( $\hat{m}(2.16), \hat{v}(2.17))$. The converted momentum operators are updated to the parameters in proportion to the given learning rate, $\alpha . \beta_{1}$ and $\beta_{2}$ are exponential decay rates. The momentum parameter assures that the objective function value is not trapped in local minima.Compared to other gradient descent algorithms, ADAM makes sure that the solution approaches a global minimum efficiently.

Along with the gradient descent algorithm, genetic algorithms are widely used optimization methods in AI research. This method mimics genetic evolution of biological systems.[38] First, we define an objective function that evaluates the fitness of the model function. Next, some of the multiple parameters are randomly selected and are exchanged or transformed. Next, if the objective function value decreases after the change of the selected parameters, the change is accepted. If the value of the objective function increases, the change is not accepted. The genetic algorithm repeats these steps numerous times to find optimal parameters. Unlike the gradient descent algorithm, because randomness is included in the variable selection, it is difficult to expect the same result from genetic algorithm when the same calculation is repeated. It is mainly used in optimization fields that do not require global minimum validation and need to reduce computational cost.

\subsection{Image Deconvolution}

In this section, using regression analysis and optimization methods, I will describe the widely used classical Lucy-Richardson algorithm and the recently developed deconvolution algorithms, ThreeDeconv and SUPPOSe. The Lucy-Richardson algorithm is the most widely used deconvolution method, while ThreeDeconv was developed to reconstruct noisy 3D wide-field fluorescence images. Recently, SUPPOSe was developed using the concept of expressing an image by a superposition of a vast amount of dispersed emitters. I will introduce these methods in conjunction with sparse deconvolution used for single-molecule localization microscopy technique. 


\subsubsection{Lucy-Richardson}

The Lucy-Richardson algorithm [67, 91] is one of the most widely used deconvolution techniques. This algorithm uses a statistical approach based on Maximum Likelihood Estimation (MLE) [24], which is a method of estimating the parameters of a probability distribution by maximizing the likelihood function. By correctly defining a likelihood function that takes into account both image formation via point spread function convolution as well as shot noise and additive image noise, this technique restores an image from data.

In order to use the Lucy-Richardson algorithm, we have to define a likelihood function and to maximize the function's value. A log-likelihood function is defined based on the probability distribution equation of the image. When an image is recorded, all pixels on a camera are statistically independent of each other. The signal of each pixel follows a Poissonian distribution, and the probability distribution over the entire image is equal to the product of the individual probabilities. Therefore, the probability distribution equation of the entire image can be written as:

$$
P_{p}(Y \mid X)=\prod_{m} \frac{(H \otimes X+G)_{m}^{Y_{m}} e^{-(H \otimes X+G)_{m}}}{Y_{m} !}
$$

A negative log-likelihood function $L(X)$ is obtained by taking the negative logarithm of the probability distribution $P_{p}$. In order to maximize $L(X)$, the derivative of the function (equation 2.21) needs to become zero. The $X^{*}$ in equation 2.22 is the solution of $\nabla L(X)=0$.

$$
\begin{gathered}
L(X)=\sum_{m}\left((H \otimes X+G)_{m}-Y_{m} \ln (H \otimes X+G)_{m}+\ln Y_{m} !\right) \\
\nabla L(X)=1-H^{T} \otimes \frac{Y}{H \otimes X+G} \\
X^{*}=X^{*} \cdot H^{T} \otimes \frac{Y}{H \otimes X^{*}+G}, \quad X^{*} \geq 0
\end{gathered}
$$


In order to find the minimum of $L(X)$, iterative calculation of $X$ is used (equation 2.23). The Lucy-Richardson technique tries to find closest value to the maximum of the likelihood function. In equation $2.23, X^{k}$ converges gradually to the closest value of likelihood as k increases. [15]

$$
X^{(k+1)}=X^{(k)} \cdot H^{T} \otimes \frac{Y}{H \otimes X^{(k)}+G}
$$

This method's advantage is that the result is good compared to the computational cost, and it is able to obtain reliable results at a high iteration number. Therefore, this algorithm is still widely used for three-dimensional image deconvolution, and research to reduce the required iteration number was reported very recently.[50]

\subsubsection{ThreeDeconv}

(a)
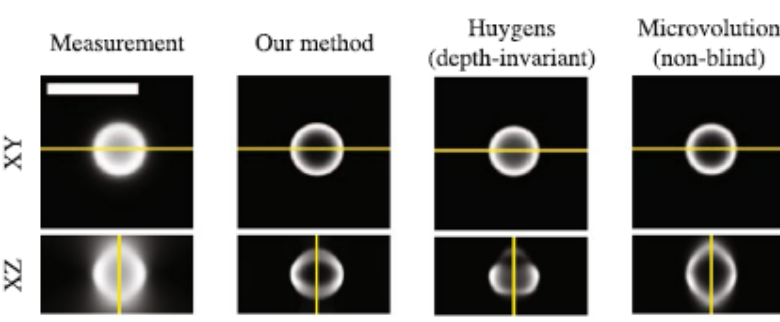

Decon.Lab2
(Richardson-Lucy)

ER-Decon 2

(b)
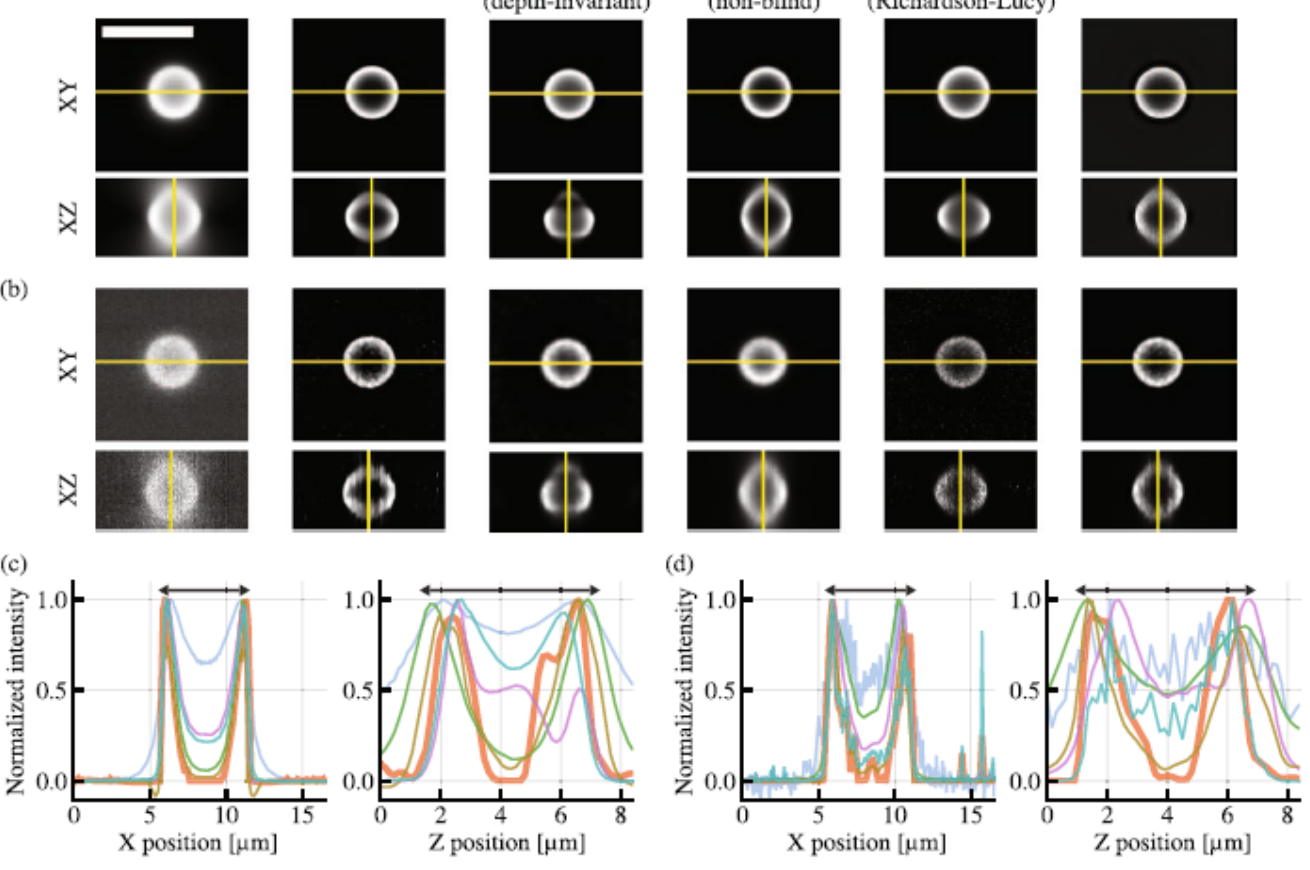

Figure 2.15: Deconvolution result using ThreeDeconv. Hayato et al. deconvolved the image of a $6 \mu \mathrm{m}$ bead. To compare the deconvolution performance, they compared several types of programs with each other. The result of ThreeDeconv showed excellent performance under low SNR.[59] 
A new deconvolution for three-dimensional wide-field microscopes has been published, reflecting the recent advances in computational technology. ThreeDeconv is a program that deconvolves images under low SNR conditions that Lucy-Richardson cannot solve. The program uses a Hessian matrix as a penalty.[59] The paper about ThreeDeconv was published in 2018 by Hayato et al., and the program uses a penalized regression method consisting of the following equation:

$$
\begin{aligned}
& \Phi=l(X)+v R(X)+I(X) \\
& l(X):=\sum\left((A X)_{j}-\left(Y_{j}+\sigma^{2}\right) \log \left((A X)_{j}+\sigma^{2}\right)\right) \\
& R(X):=\sum\left\|H_{j} X\right\|_{F}
\end{aligned}
$$

$$
H f(x, y)=\left[\begin{array}{cc}
\frac{\partial^{2} f}{\partial x^{2}} & \frac{\partial^{2} f}{\partial x \partial y} \\
\frac{\partial^{2} f}{\partial x \partial y} & \frac{\partial^{2} f}{\partial y^{2}}
\end{array}\right]
$$

$v$ is an weighting factor for $R(X)$, and $I(X)$ is an indicator function, which is zero if all elements of the input are non-negative and infinity otherwise. $\sigma$ is the read noise standard deviation. $A$ is the linear image formation operator representing the three-dimensional convolution with the microscope's PSF. The characteristic part of the objective function is the Hessian matrix in the penalty parameter, $R(x) . R(x)$ is composed of the Frobenius norm of the Hessian matrix. The Hessian matrix is a square matrix composed of the second partial derivatives, see equation 2.27, and the Frobenius norm is defined by the following equation:

$$
\|A\|_{F}=\sqrt{\sum a^{2}}
$$

where the $a$ are the singluar values of $A$. The Hessian matrix measure the second derivatives of a function. Due to the second derivatives, this matrix is sensitive to 
brightness changes of the image. In ThreeDeconv, by limiting the size of the Hessian matrix, a smoothing effect on images is achieved, and as a result, we obtain an image with reduced noise.

\subsubsection{Sparse Deconvolution}

Unlike Lucy-Richardson and ThreeDeconv described above, there are also deconvolution programs designed to analyze images of single molecule localization microscopy (SMLM). These programs use compressed sensing, a technique that separates each signal when the signal meets sparsity and incoherence condition. [27, 35] Deconvolution programs based on compressed sensing analyze SMLM images of high density fluorophores that cannot be localized by Gaussian fitting. This method is called sparse deconvolution because it uses sparse regression under the condition that satisfies the sparsity premised on compressed sensing, and programs such as CSSTORM, FALCON, and SPIDER use this method.

As described above (see 2.3.2), when the power of the penalty parameter is less than or equal to 1 , the sparsity of the solution increases. This phenomenon is advantageously used for sparse deconvolution. The first program to use sparse regression was CSSTORM, which is a deconvolution method using L1 penalized regression so called LASSO regression. [122] FALCON also uses the same regression analysis, but this deconvolution program uses an additional algorithm that implements sub-grid resolution. [77]

Unlike CSSTORM and FALCON, a program that uses L0 penalty regression, SPIDER, was developed. An indicator function was used as the L0 penalty parameter. [57, 58] The indicator function value becomes 1 if each pixel has an emitter and 0 otherwise. That is, the penalty parameter in SPIDER is physically proportional to the amount of point emitters distributed in the image. We can control the sparsity of the signal by limiting the penalty parameter within a certain range. By adjusting the parameters to match the sparsity of the fluorophores, it is possible to localize them more accurately than CSSTORM and FALCON. CSSTORM, FALCON, and SPIDER using sparse regression show high applicability and better results for SMLM compared to other deconvolution programs. 
a)

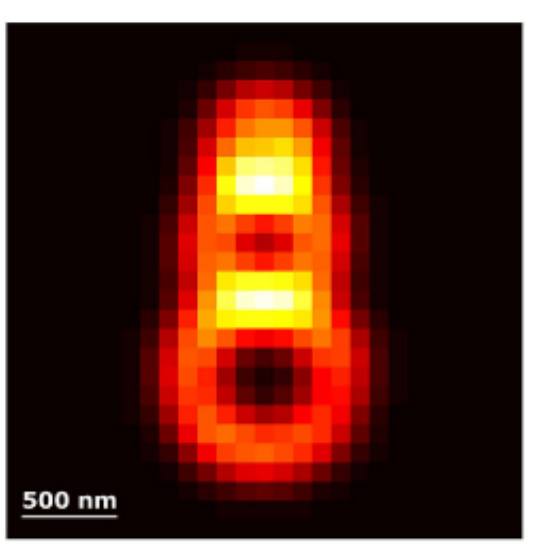

c)

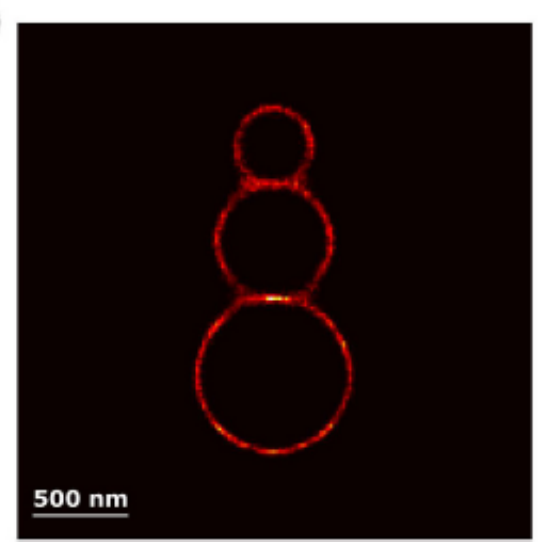

b)

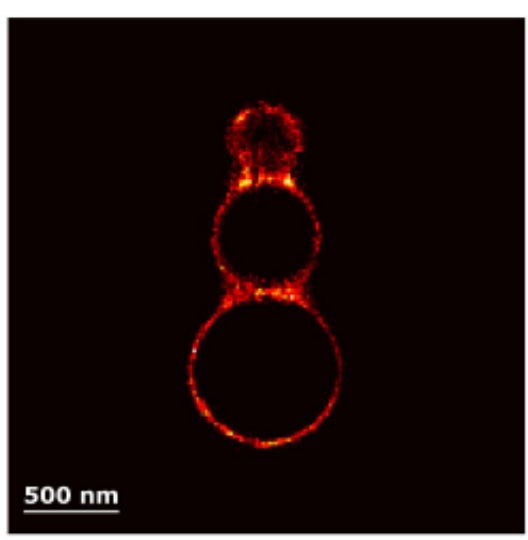

d)

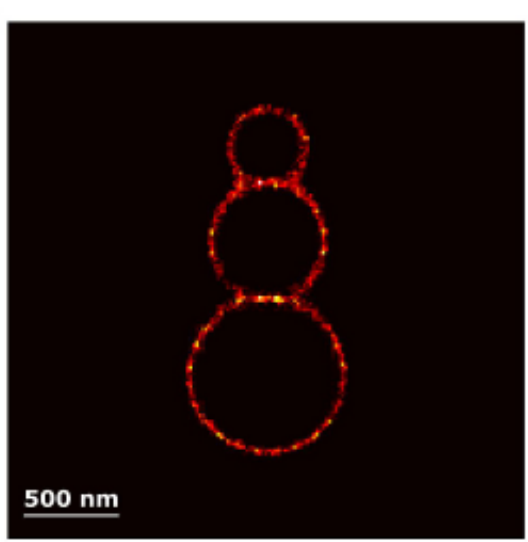

Figure 2.16: The artificial ring image was deconvolved with three different sparse deconvolution programs. (a) is the original image, and (b), (c), (d) are the deconvolution results of CSStorm, FALCON, and SPIDER. [57] (this figure is licensed under CC BY 4.0 [57])

\subsubsection{SUPPOSe}

Even in the case of sparse deconvolution, it is difficult to deconvolve an image under extremely high density of fluorophores like a single frame of general fluorescence image rather than an SMLM image set. The accuracy of sparse deconvolution increases when adequate sparsity is maintained. In SMLM, a single fluorescence image is reconstructed by recording and processing numerous images that meets the sparsity condition. However, by using sparse deconvolution methods, it is difficult to calculate the position of each fluorophore distributed in a general image that contains a large number of fluorophores. Since the fluorescent molecules in the image are almost continuously distributed, we cannot estimate the position of the fluorophores one by one from the continuous signal. 
Although the fluorophores seem to be continuously distributed in an image, the signals are not continuous. Molecules are distributed discontinuously at different positions no matter how densely distributed it is. In sparse deconvolution, a penalty parameter is used to enhance the sparsity of the calculation result, but there is a certain range of values that the parameter can have. In some cases, in order to lower the value of the objective function, the penalty parameter is excessively lowered, or conversely, the constrain on the value does not work properly. As a result, in a deconvolution result of some images, the resulting image can be still blurred after deconvolution, or the deconvolution can result in an excessively discontinuous image.

To solve this problem, a deconvolution program that strongly limits the number of fluorophores was developed. This deconvolution program, known as SUPPOSe, was published in 2019 by Martinez et al. [73] This program uses the method of optimizing the positions of a fixed number of virtual fluorophores. By limiting the overall brightness and placing a limited number of virtual fluorophores having unit intensity, a continuous image is considered as a sparse data. In this process, the program optimizes every position of the virtual fluorophores together, and the image was reconstructed based on the positional information that was optimized.

The calculation process of the program is as follows:

$$
\begin{aligned}
& X(x)=\alpha \sum_{k=1}^{N} \delta\left(x-a_{k}\right) \\
& S(x)=H \otimes X(x)=\alpha \sum_{k=1}^{N} P S F \otimes \delta\left(x-a_{k}\right)=\sum_{k=1}^{N} I\left(x-a_{k}\right) \\
& \chi^{2}=\|Y-S\|^{2}
\end{aligned}
$$

First, the program defines the virtual emitters having equal brightness as in equation 2.29. The image, $X$ is reconstructed through the super-position of $N$ delta functions. Each scaled delta function denotes one individual virtual emitter, and $N$ is total number of the emitters. $X$ is a map of the emitters that is not blurred. By convolution with the PSF, a virtual image, $S$, is reconstructed. (equation 2.30) After that, we optimize the positions of every virtual emitter by minimizing the objective function in equation 2.31 . The objective function is designed to maximize the similarity between the measurement results, $Y$ and the constructed image, $S$. In the paper about SUPPOSe, the results shown in figure 
2.17 showed that accuracy was not good comparing the direct localization of the single point source, but the resolution was improved considerably than before deconvolution.

(A) Point source \& PSF

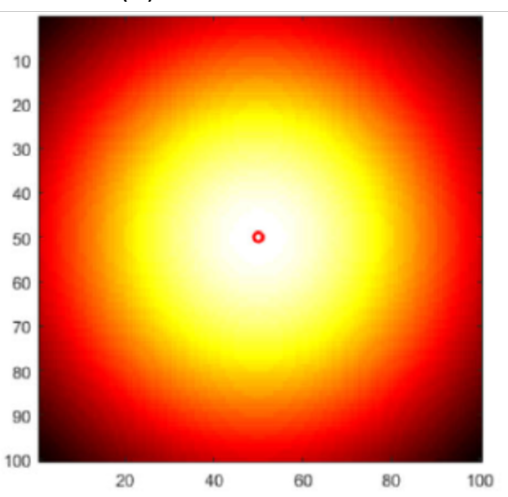

(B) Point source \& localization

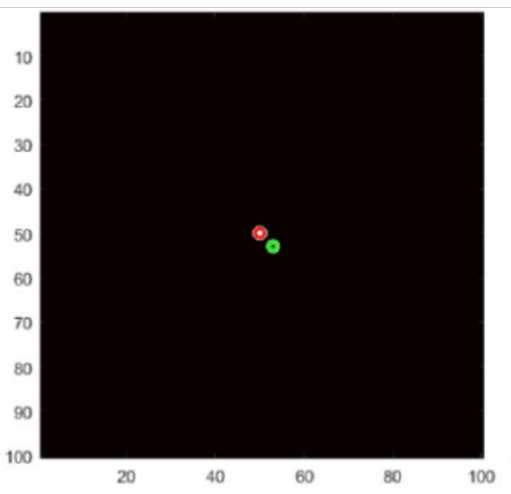

(C) Point source \& SUPPOSe

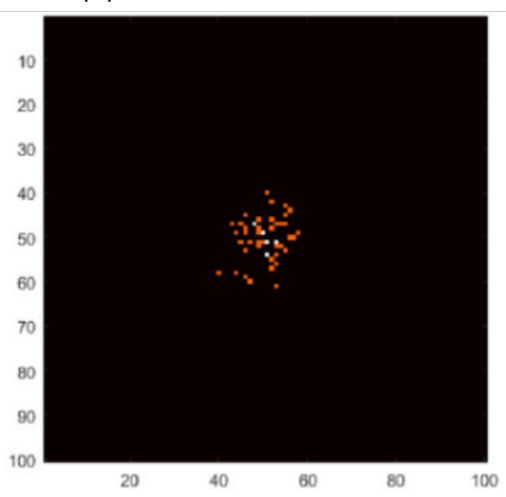

Figure 2.17: Deconvolution result of one virtual single emitter. The deconvolution result is composed of multiple virtual spots. [73]

\subsection{Analysis methods for fluorescence microscopy}

The above described methods for image reconstruction are at the basis of this thesis. Based on these methods, we want to obtain new information from measured threedimensional images. New information does not come from the rendered 3D image itself, but when a physical interpretation is applied to the image. Therefore, in the next sections, I will explain how to get physical information from images. These methods will be used in Chapters 4 and 5 to describe the motion of fluorescent molecules.

\subsubsection{Center of fluorescence mass and geometric center}

In order to obtain physical information from a fluorescence image, it is essential to define the center of an object in an image. To extract the motion of a specific object from a sequence of fluorescence images, we need to find the position of the object for each time point. The position of the object is described as a point representing the distribution of the material which the object consists of. Based on the distribution of the fluorescence signal in an image, we can estimate the center of the object. A fluorescence movie is expressed as a stack of images along the time axis, and by defining the center of the object in an image at each time step, we can determine the positional change of the object. For this purpose, we can use several methods like finding positions of intensity maximum, centroid, or center of mass. The most widely used method is to find the 
position of maximum intensity by fitting a Gaussian function. This method is mainly used for small-sized particles, and the method is used as a localization method in SMLM. However, when the size of the object is large or the shape is complex, it is difficult to use this method because it is difficult to define the shape of the object with a simple Gaussian function. Therefore, for an object having a large or complex structure, a method using a center of mass or a geometric center is mainly utilized. [48, 61, 119]

The centroid corresponds to the the arithmetic mean position of all the points in the object. To define a centroid, we assume uniform density over the entire volume. It is described by the formula as follows:

$$
\sum_{i=1}^{n}\left(r_{i}-R\right)=0
$$

In this equation, $R$ is the center position of the object, and when the sum of distances from each point $r_{i}$ becomes 0 , this point is called a geometric center or centroid. Considering the influence of the density previously assumed in the above equation, the equation is transformed as follows:

$$
\sum_{i=1}^{n} m_{i} \times\left(r_{i}-R\right)=0
$$

If the effect of mass, $m_{i}$, on the distance from the center point is considered as the above equation, the point, $R$ becomes the center of mass. The difference between the centroid and the center of mass is the effect of density. Therefore, by comparing the change of the centroid with the change of the center of mass, we can find out not only the visualized motion of an object but also the flow of matter inside the object. However, from the fluorescence image we have no way to determine the exact density of the whole material. Instead, the density of the fluorescent molecule can be inferred from the fluorescence image by using the characteristics of fluorophores. The same fluorophores have the same mass and fluorescence intensity. Since the fluorescence intensity is proportional to the mass of the fluorescence molecule, the formula for the center of mass can be modified as follows:

$$
\sum_{i=1}^{n} I_{i} \times\left(r_{i}-R\right)=0
$$


In this equation, the mass, $m_{i}$, is replaced with the fluorescence intensity, $I_{i}$. Although information about other substances except fluorescent molecules is unknown, this method helps to understand the movement of fluorescent molecules over time, and this method was used in 2017 to understand the flow of molecules in cells, and the authors of the paper about the methdo named it as the center of fluorescence mass. [105] The center of fluorescence mass, center of mass, and centroid can be used to provide information on the overall movement of cells and the movement of internal substances over time.

\subsubsection{D Optical Flow Estimation}

Centroid and center of mass can be used to explain the overall movement of the cell or the average movement of fluorescent molecules inside the cell. However, when molecules move within a cell, it is difficult to obtain local information with methods determining average changes of the entire cell. Here, I introduce the optical flow estimation method, which allow us to calculate local distribution changes over time.

Optical flow is the pattern of apparent motion of objects, surfaces, and edges in a visual scene caused by the relative motion between an observer and a scene[14, 55], and this concept was introduced around 1940 by James J. Gibson.[46] When the brightness of the image $I(x, y, z, t)$ varies with time, assuming the brightness change to be small, the image after the time $\Delta t$ can be approximated using a Taylor series:

$$
I(x+\Delta x, y+\Delta y, z+\Delta z, t+\Delta t) \simeq I(x, y, z, t)+\frac{\partial I}{\partial x} \Delta x+\frac{\partial I}{\partial y} \Delta y+\frac{\partial I}{\partial z} \Delta z+\frac{\partial I}{\partial t} \Delta t
$$

The brightness change of the image can be summarized as:

$$
\begin{aligned}
& \frac{\partial I}{\partial x} \Delta x+\frac{\partial I}{\partial y} \Delta y+\frac{\partial I}{\partial z} \Delta z+\frac{\partial I}{\partial t} \Delta t=0 \\
& I_{x} V_{x}+I_{y} V_{y}+I_{z} V_{z}+I_{t}=0
\end{aligned}
$$

The problem to be solved in optical flow estimation is to find the velocity $V$ that satisfies equation 2.37 .

This concept has been widely used for image processing in the context of psychology, robotics, and biology. In the last 20 years, results of optical flow estimation using various 
algorithms have been published to analyze the three-dimensional flow of molecules within cells[9, 20, 21, 56, 71]. Among them, two algorithms that showed good results, the coarse-to-fine warping method and the PatchMatch algorithm, will be discussed here.

The coarse-to-fine warping method is an algorithm utilizing image deformation. This method estimates the overall flow at varying spatial sampling frequencies. In order to describe the motion of an object, a sufficient temporal sampling rate is required. When the temporal sampling rate is insufficient, the aliasing described in 2.2.1 occurs, and "Coarse-to-fine" processing strategies have been developed to deal with the temporal aliasing problem. [20, 68, 75] These algorithms are typically implemented using an "image pyramid", which is a type of multi-scale signal representation.[5] The motivation for this approach is that aliasing only affects the high spatial frequency spectrum of the input sequence. Therefore, the speed of an object can be accurately estimated from the downsampled image with a low-pass filter. The calculational result of the downsampled image can be used as an initial guess for estimating motion at a finer scale. Alternatively, aliasing in the fine-scale image can be removed using the speed estimated on a coarser scale. Finally, the velocity field of the warped image is calculated, and the velocity field on a coarser scale can be added as a correction term to the calculation of the original image.[20]

Another method used for optical flow estimation of intracellular molecules is the PatchMatch algorithm. The PatchMatch algorithm was reported in 2009 by Barnes et al.,[13] and the algorithm finds the best matching pair, $\left(P_{c}\right)$ through the displacement of a patch $(P)$, which is a part of the image. The distance between $P$ and $P_{c}$ is used to calculate the velocity field in optical flow estimation.[13] This method performs parallel calculations for each unit patch constituting an image. Neural network programming and GPUs enable us to efficiently perform the parallel computations of this algorithm. Therefore, recently, many papers describing cell movement using this method have been published.[71] 

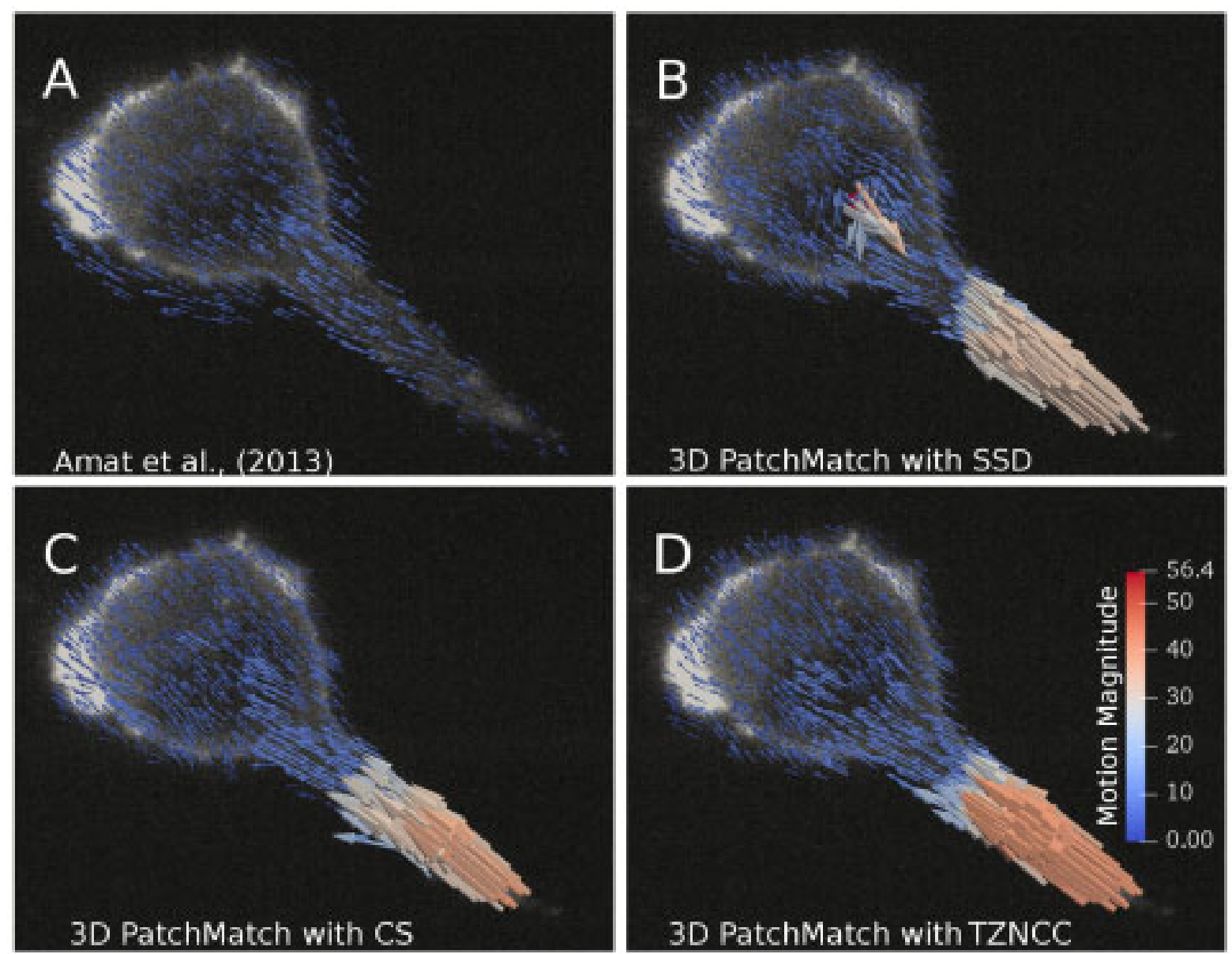

Figure 2.18: Comparison of flow estimation results. The results using the PatchMatch method with three different objective functions were compared with coarse-to-fine warping flow estimation result of Amat et al.[9, 71] 



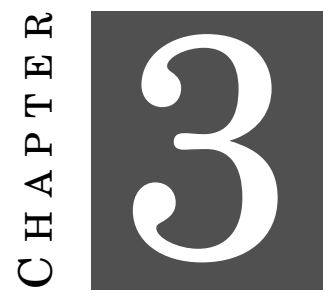

\section{IMAGE ANALYSIS METHODS FOR} MULTI-PLANE MICROSCOPY

his chapter describes the image analysis methods used in this study. The microscope

I I used in this research is a multi-plane setup, and this chapter introduces methods for reconstructing multi-plane microscopy datasets into 3D images. As described in 3.1.1, a calibration method has been introduced to correct brightness variations across image plans and to calibrate the inter-plane distance. The most important information for the calibration method is the maximum intensity and z-position of the fluorescent beads. Since the accuracy of these two values is the most important factor to render a precise three-dimensional image with a multi-plane setup, a potential error in the calibration will change the experimental results and their explanations. Therefore, it is important to reduce potential errors. After precise calibration, the three-dimensional distribution of fluorescent molecules can be visualized. However, due to the missing cone problem in wide-field imaging, the image is blurred along the z-axis. The problem makes it difficult to accurately describe the axial distribution of fluorophores. To solve this problem, I introduce a new deconvolution program. Then, an optical flow estimation program is used to track the position of molecules over time from the deconvolved three-dimensional movies. Calibration, deconvolution, and optical flow estimation methods are introduced and their accuracy is evaluated. 


\subsection{Method}

\subsubsection{Calibration of the Multi-plane Setup}

As described above (see 2.1.5), the image data obtained by the multi-plane setup consists of eight two-dimensional images. Images from different focal planes reach the image sensor at the same. To reconstruct these images into 3D images, we need to know the distance between each focal plane and the relative brightness of each image. The inter-plane distance determines the z-axis size of every voxel, and when the brightness between each two-dimensional image is uniform, the shape of the image distributed in the three-dimensional space is ideally expressed.

In a prism based multi-plane setup, the brightness of each image plane is theoretically the same, and the inter-plane distance can be calculated theoretically using the equation 2.6. The inter-plane distance is determined by the magnification of the objective lens and the distance between the beam splitter and the mirror. When using a prism based multi-plane setup, this distance is fixed. The brightness of each image plane is divided by a beam splitter, and the beam splitter ideally splits the beam with an intensity of 50:50. The mirror used also has a reflectivity close to $100 \%$. Therefore, theoretically, the brightness of all image planes should be identical, and the distance between the planes should be accurately predicted.

However, in actual measurement conditions, the brightness of each plane is not identical. Depending on the sample medium, the optical path can be changed, and some errors can occur in the alignment of the actual mirrors. Beam splitters also have an error in the ratio between the reflected and transmitted beams depending on the wavelength. Also, the actual inter-plane distance of the setup can deviate from the theoretical numbers. If this is not corrected, the size of the object reconstructed in three dimensions will be distorted. Errors in the intensity ratio also change the shape and size of the measurement results. Therefore, it is necessary to accurately correct the inter-plane distance and the brightness ratio between each image plane.

In the first paper of the multi-plane setup, a calibration method using fluorescent beads was introduced to correct the error in the brightness ratio. [44] The fluorescence intensity of each particle was measured by dispersed fluorescent beads on a plane and moving the sample vertically at $500 \mathrm{~nm}$ intervals (see figure 3.1). The image of the bead placed in the focal plane has the maximum intensity. The intensity change according to the 
vertical position of the bead was measured in each image channel, and the position of the maximum intensity was measured. Through this process, each position of focal planes was measured and the inter-plane distance was calculated. This method is mainly used in research related to multi-plane imaging, and the method has become a standard calibration method for the technique.

a
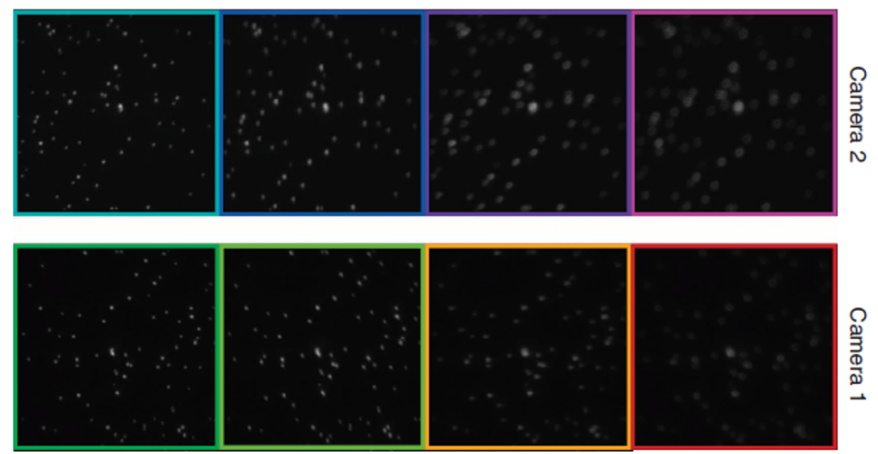

b
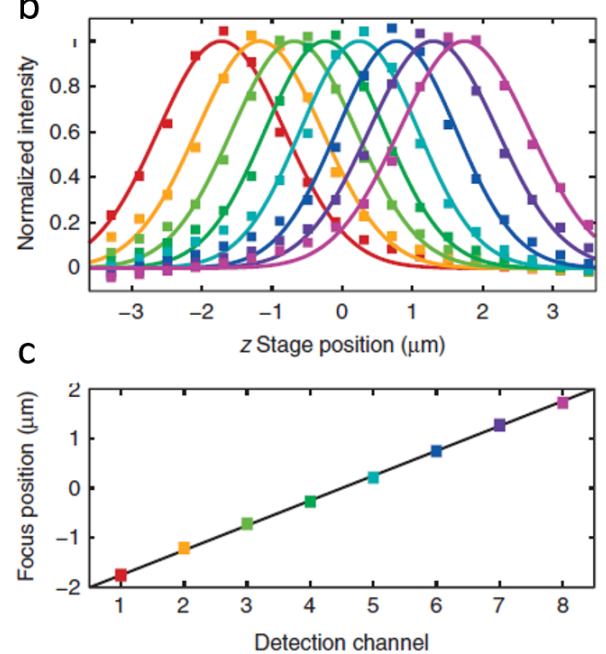

Figure 3.1: The calibration method of multi-plane setup. Images of fluorescent beads in different image planes (a). When we scan the sample along the z-axis, we can record the intensity change like (b). The positions of maximum intensity are the positions of the focal planes. We can define the interplane distance based on the distances between these peaks (b). The plot in (c) shows the distances between the image planes. Before we normalize the intensity in (b), we can also calculate the intensity ratio between image planes by comparing maximum intensities. [44]

\subsubsection{Intensity Calibration of the Individual Imaging Planes}

In the method using fluorescent beads 3.1.1, the precision of calibration is affected by the fluorescence brightness fluctuation or photobleaching. If the measurement time for the fluorescence signal becomes longer, the brightness naturally decreases by photobleaching. Photobleaching changes the brightness of the sample, which must remain uniform during the whole measurement time. Although it is possible to reduce the photobleaching effect by minimizing illumination, it is not a fundamental solution. The instability of the fluorescence brightness changes in the brightness ratio between image planes, and it affects also the calculated value of the inter-plane distance. The vertical position of the focal plane is calculated as the point where fluorescent beads shows maximum intensity, 
but this method is applicable only when the brightness of the bead is uniform in all measurement conditions. Photobleaching effect can shift the center of the brightness distribution in Gaussian function in figure 3.1. Therefore, the estimated vertical position of the focal plane can be changed, and as a result, the inter-plane distance is also changed. Here, I introduce a method how to correct the error considering the structural characteristics of the beam-splitter and the effect of photobleaching.

First, I will explain the cause of the non-uniform inter-plane intensity ratio as follows. Theoretically, the beam splitter inside the prism should split the beam with a 50:50 brightness ratio. When all the light passing through the beam splitter is equally split, the brightness of all image planes becomes the same. However, practically, the ratio at which the beam splitter splits the brightness is slightly different depending on the wavelength. The difference in error rate according to these wavelengths changes the brightness of the image plane. In order to understand how much the brightness ratio is changed, the brightness ratio of a single beam splitter is expressed as an equation as follows.

$$
\begin{aligned}
& I=I_{r}+I_{t} \\
& I_{r}=I \times(0.5-\epsilon) \\
& I_{t}=I \times(0.5+\epsilon)
\end{aligned}
$$

If the difference in brightness ratio between the reflected beam $\left(I_{r}\right)$ and the transmitted beam $\left(I_{t}\right)$ is calculated as above, the equation in the case of being reflected or transmitted through the beam splitter several times can be defined as follows.

$$
I_{N}=I \times(0.5+\epsilon)^{r}(0.5-\epsilon)^{t}
$$

Assuming that the mirror's reflectance on the outside of the prism is almost perfect, the number of interactions with the beam splitting part is the critical parameter. Relative brightness is defined based on the number of interactions ( $r$ and $t$ ) between the images and beam splitter in the prism. One fits the brightness data with the equation 3.4. We can also measure the error value of the beam splitter from the fitting result. 
Using the equation 3.4, we can measure the magnitude of the error that prism has. The magnitude of the error varies depending on the wavelength. Therefore, it is most ideal to measure the spectrum. However, in this study, the wavelength-dependent error was minimized by limiting the range of the measurement wavelength according to the wavelength range of the fluorescence signal in live cell measurements. The filters used in the measurement were Quad Line Beamsplitter R405/488/561/635, Semrock and a band-pass filter 520/45, Semrock.

a

White light (Halogen lamp)

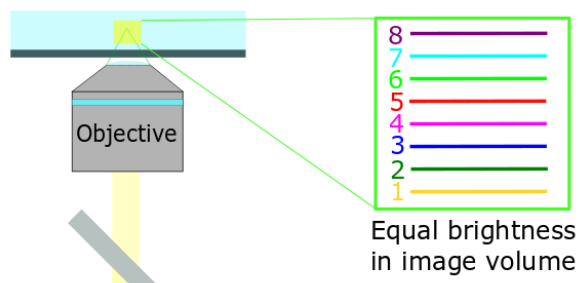

Multi-bandpass dichroic

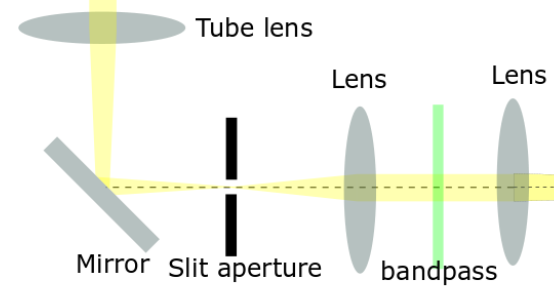

b

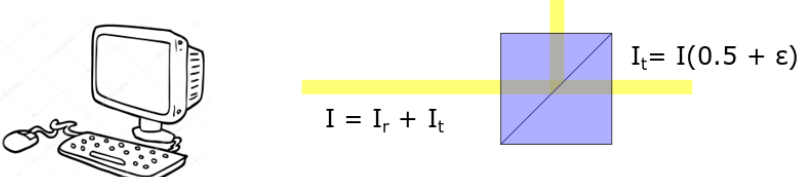

Intensity analysis based on model equation

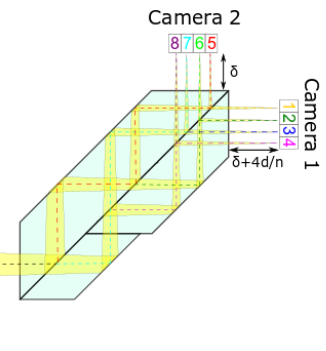

C

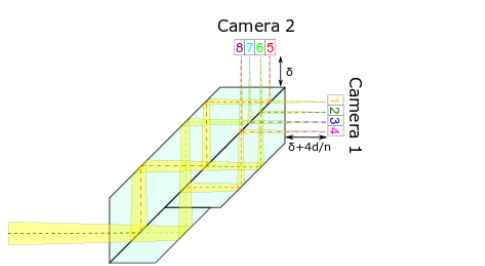

$I_{\#}=I(0.5+\varepsilon)^{t}(0.5-\varepsilon)^{r}$

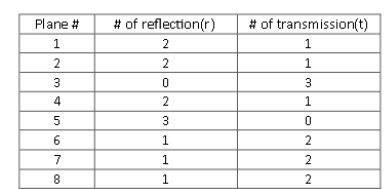

Figure 3.2: (a) Image volume is filled with white light by a halogen lamp. The constant image brightness on every image plane is filtered with the fluorescent filter unit. The images on every plane are separated by the prism. The images measured by two cameras are analyzed based on the equation considering the structure of prism. (b) The intensities of the reflectance and transmitance beams can be calculated based on the equation. ( $\epsilon$ is error value of intensity ratio.) (c) Based on the structure of the prism, we can set the equation of relative intensity. The number of reflectance and transmittance through the beam splitter is in the table.

The correction method of relative intensity based on the structure of the prism can reduce the irregular intensity problem. However, when we measure the intensity value with small error, we can correct the values more precisely using the above equation. Therefore, it is also important to remove the effect of photobleaching on relative intensity. In this study, the effect of photobleaching was eliminated by comparing the brightness of each focal plane using white light. Using a halogen lamp, a wide field-of-view was filled with 
light of constant brightness, and then the brightness of each image plane was measured. The brightness of each image plane was measured simultaneously for 1 second, and the relative brightness of each image plane was compared with the average value of the brightness of the central part. The figure 3.2 describes the measurement scheme.

\subsubsection{Deconvolution Method by Superposition of Dispersed Points (3D-SUPPOSe)}

After correcting the 3D image with the new calibration method, deconvolution method was used to reduce the effect of the missing cone problem, which is a disadvantage of widefield illumination. In this study, SUPPOSe[73], which is known to have high accuracy among various deconvolution programs, was improved to be suitable for processing multi-plane setup data.

A lot of computational resources are needed to analyze the 3D image data. For example, compared to a $2 \mathrm{D}$ image composed of $512 \times 512$ pixels, a $3 \mathrm{D}$ image composed of $512 \times 512 \times 8$ voxels, which is the general size of a multi-plane setup data, consumes 8 times more memory. That is, the capacity is multiplied by the number of voxels along the z-axis. If the aspect ratio of the voxel is adjusted to appear uniform in all directions, the memory consumption becomes larger due to the effect of upsampling along the z-axis performed to increase the axial sampling rate. This data size increase is simply a comparison of a single image. When we consider the increase in additional memory required for the convolution process, the memory consumption is increased multiple times more. Therefore, utilizing a limited size of memory is very important for the efficient $3 \mathrm{D}$ image deconvolution.

SUPPOSe has one more cause that increases memory consumption. In other deconvolution programs, the brightness array of the image becomes a variable, so only the size of the image needs to be considered as a variable size. However, since SUPPOSe reconstructs an image by combining massive virtual emitters, memory is consumed additionally to store the positional information of each emitter. In order to more quantitatively calculate the amount of memory generated in this process, it is assumed that SUPPOSe uses 10 million virtual fluorophores. In the case of storing positional information as 32-bit data, 30 million integers must be separately stored in variables of 10 million emitters, and for this purpose, more than 100 Mbyte of memory is consumed. The number of emitters can be increased because it changes according to the average 
brightness and intensity variation of the image, and it is not difficult to exceed the several Gbytes memory when we consider the size of the temporal variables generated in the program. Therefore, efficiently controlling the memory usage is an important problem to be solved, in order to make the SUPPOSe algorithm more efficiently.

To modify SUPPOSe into a more practical algorithm for 3D images, I utilized the independency of each virtual fluorophore signal. SUPPOSe reproduces images composed of independent virtual fluorophores. The image is reconstructed based on the combination of massive virtual emitters that are independently distributed in space. Therefore, we can also perform position optimization for each emitter separately. For example, when optimizing the positions of 10 million emitters, we can make 100 subsets of 100,000 emitters. Using the subsets, we perform 100 individual deconvolutions and recombine 100 results. With this method, the memory usage can be reduced by about $1 / 100$. However, in this case, it is necessary to remove the number of emitters that have already taken into account in the previous deconvolution process. Therefore, it is necessary subtract their contrubution from the original image. This method is explained as follows.

$$
\begin{aligned}
& N=t \times n \\
& X(x)=\sum_{l=1}^{t} X_{l}(x)=\alpha \sum_{l=1}^{t} \sum_{k=1}^{n} \delta\left(x-a_{l k}\right) \\
& S_{l}(x)=P S F \otimes X_{l}(x)=\sum_{k=1}^{n} I\left(x-a_{l k}\right) \\
& Y_{l}=Y-\sum_{m=0}^{l-1} S_{m}\left(S_{0}: \text { array of zeros }\right) \\
& \chi_{l}^{2}=\left|Y_{l}-S_{l}\right|^{2}
\end{aligned}
$$

In this equation 3.5, the total number of emitters, $N$, forms $t$ subsets of $n$ points. Each emitter is expressed in the image as a delta function scaled by $\alpha$ of each emitter as shown in equation 3.6. A subset consisting of $n$ points, $X_{l}$, is created, and we can have the total map of emitters, X, by adding every $X_{l}$. From each map $X_{l}$, one obtains its image $S_{l}$ through convolution with the PSF.(in equation 3.7) Optimization of the map is performed by minimizing the difference between $S_{l}$ and the measured sub-image, $Y_{l}$. This process is also explained in the figure 3.3. By repeating this process $t$ times, the total map of the emitters, $X$ is optimized. In order to avoid repeated calculation of a specific emitter, as in equation 3.8, the images obtained from the deconvolution result up to the previous 
step, $S_{l}$, are accumulated and subtracted from $Y$ for next deconvolution step. Although this process has the disadvantage of lengthening the computation time through iterative deconvolution, it enables deconvolution using SUPPOSe with control of the resources of the computer hardware.

The optimization method that affects the accuracy of SUPPOSe was also changed in this study. In the paper about SUPPOSe, the authors used a genetic algorithm to minimize the objective function, but in this study, gradient descent algorithm was used. Since the gradient descent algorithm finds a minima based on the calculated gradient information, the same result is obtained even after repeated calculations. In particular, Adam, one of stochastic gradient descent algorithm described in 2.3.2, can efficiently optimize massive variables at the same time and can also reduce the possibility of being trapped in local minima by using the momentum parameter. However, there is a disadvantage in that the additional memory usage to calculate gradients is generated during the optimization process. When Adam is used, gradient and momentum parameters $m$ and $v$ of the same size as the variable size are additionally generated during the optimization process at every step. However, if we optimize variables with the gradient descent algorithm, deconvolution result is regularized in the iterative optimization method compared to the heuristic minimization method like genetic algorithm.

The genetic algorithm randomly selects variables and adjust the values at every instance, so the calculation results cannot be uniform. Of course, by limiting the termination criteria of the objective function, the result can be derived within a certain range. However, it is still difficult to confirm that the result is always the same, and the calculation time is also not uniform. However, the gradient descent algorithm changes the variable following the direction that the function value decreases based on the gradient at every moment. This characteristics of this optimization process provides a direction to the minima. In this study, we used the gradient descent algorithm to focus more on its accuracy and reproducibility. Among the gradient descent algorithms, we used Adam, which can speed up optimization for a large amount of variables and avoid local minima.

The code in this program uses Python (https://www.python.org/) and TensorFlow (https://www.tensorflow.org/) to utilize a simple convolutional neural network structure. Detailed code will be released through GitHub (https : //github.com/hongjejang/ 3D-SUPPOSe). All calculations were performed on a Xeon W-2145 CPU, 64 GB RAM, and 
NVIDIA Quadro P4000 GPU. The image data of the $6 \mu \mathrm{m}$ bead was downloaded directly from internet account[59].
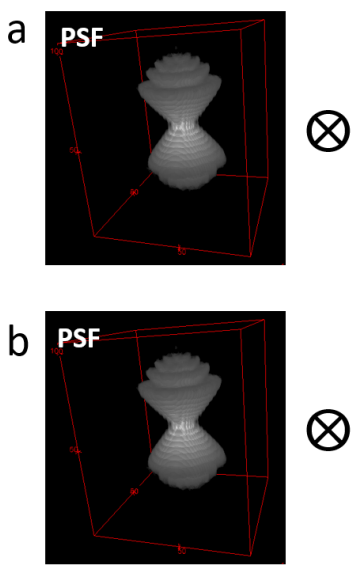

Measured image

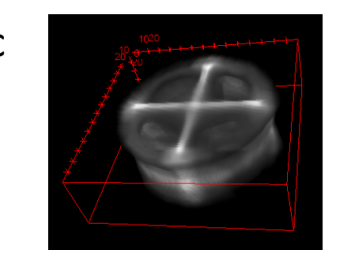

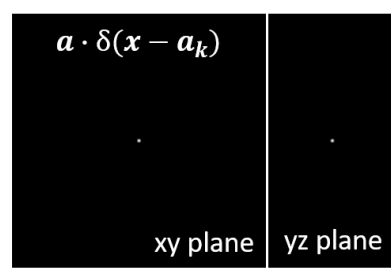
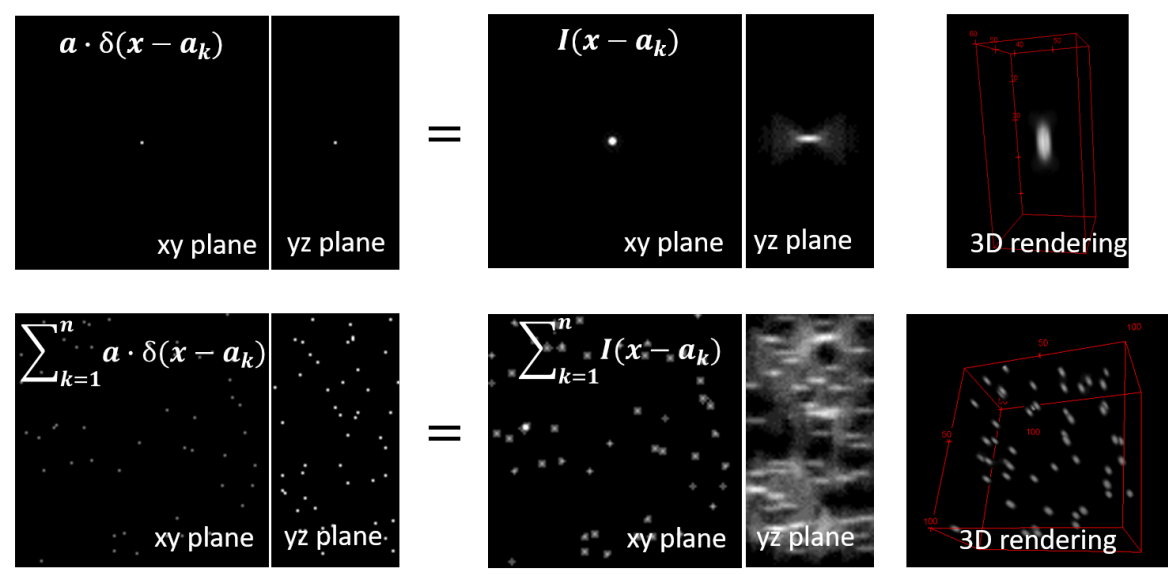

Image of virtual emitters
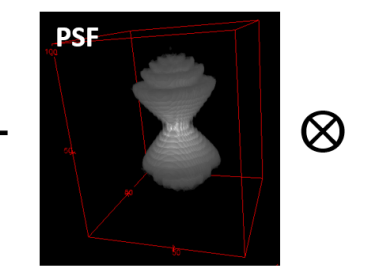

Optimize $\boldsymbol{a}_{\boldsymbol{k}}$ by minimizing the objective function

Figure 3.3: (a) Convolution process of single virtual emitter. The image of a single emitter is blurred by the PSF. (b) Convolution process of multiple virtual emitters. The multiple emitters are randomly distributed and blurred by PSF. Using this process, we can generate the blurred image of multiple emitters. (c) We can build the objective function with the image of multiple emitters and measurement data. By minimizing the objective function, we can optimize the positions of the virtual emitters.

\subsubsection{New 3D Optical Flow Estimation}

The calibration method and the 3D-SUPPOSe help to reconstruct more accurate threedimensional image from a multi-plane setup dataset. After the $3 \mathrm{D}$ rendering, we can arrange a temporal sequence of $3 \mathrm{D}$ images to compose a movie $I(x, y, z, t)$. The analysis of this data, we can reveal information about the movement of an object. The local brightness change explains the positional change of fluorescence molecules in each voxel. The signal distribution change is a result of particle movement, and the fluorescent molecules moving to the adjacent voxel results in an increase and decrease of the fluorescence signal in some voxel. As described above (chapter 2.5.2), optical flow estimation is used to describe the movement of molecules in the image of cells. In the case of the PatchMatch algorithm or the coarse-to-fine warping method, the local fluorescence change inside 
the volume is calculated, but the area is not narrowed down to a single pixel or voxel. Therefore, in this study, an optical flow estimation method through direct single pixel level calculation was performed.

a

$I_{1}$ (Frame 1)

$\mathrm{I}_{2}$ (Frame 2)
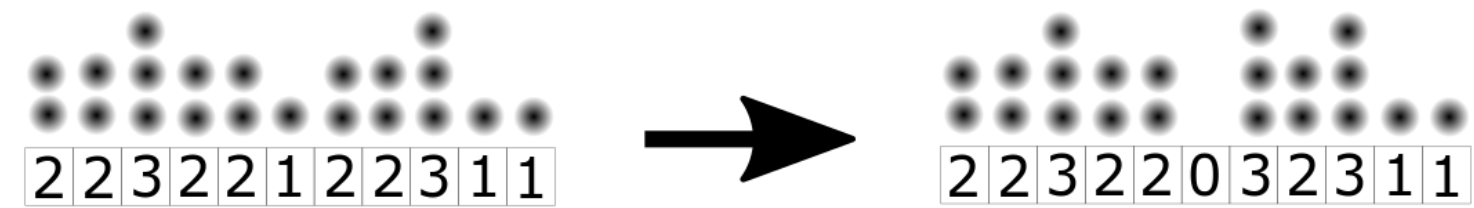

b
$\mathrm{V}_{\mathrm{x}}$
Sobel filter $\quad \Delta I=I_{2}-I_{1}$
$00000100000 \otimes 1-10=00000-110000$

C

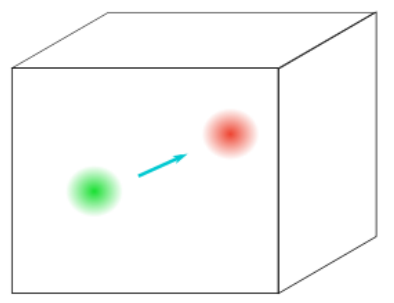

$$
\begin{aligned}
& \text { objective function }= \\
& \left|\Delta I-V_{x} \otimes u-V_{y} \otimes V-V_{z} \otimes w\right|
\end{aligned}
$$

Figure 3.4: a) Intensity of 1 dimensional image strap is changing after certain time. b) The strap of the moved particle number to right side is convolved with modified Sobel filter. The result of convolution is the same value with the difference between two frames. c) By setting the objective function for 3-dimensional image analysis, we can calculate the number and direction of intensity movement between time frames.

To explain the basic concept how to calculate the fluorescent molecular flow, let us consider a simple one-dimensional model. We assume that a particle moves from one pixel to an adjacent pixel in a one-dimensional array. As shown in figure 3.4, if one particle moves to the adjacent pixel on the right side in the image of $I_{1}$, the resulting image becomes equal to $I_{2} . I_{1}$ and $I_{2}$ are images describing that one particle moves from pixel 5 to pixel 6 after a unit time. Using the basic equation of optical flow estimation, the change of fluorescence intensity between two frames can be explained using velocity and intensity change over time. We can define the effective velocity the fluoreophores in 
each pixel as an array having same dimension of the image. The change in brightness $\left(\Delta I=I_{2}-I_{1}\right)$ is expressed through convolution between the velocity array and the gradient array, $\frac{\partial I}{\partial x}$. Here, the gradient $\delta I / \delta x$ is approximated be the Sobel filter operator, which is in the one-dimesional case simply the vector [ 1010$]$.

In order to analyze a 3D image with the same concept, the objective function is extended to $3 \mathrm{D}$ as shown in panel $\mathrm{c}$ of figure 3.4. Since $I$ has information about the $x, y$, and $z$ axes, we add convolution results between the velocity $\left(V_{x}, V_{y}, V_{z}\right)$ and the derivative filter $(u, v, w)$ for each axis.

$$
\Phi=\left|\Delta I-V_{x} \otimes u-V_{y} \otimes v-V_{z} \otimes w\right|
$$

As shown in the above equation, the difference between the convolution between $\Delta I$ and the velocity for each axis $\left(V_{x}, V_{y}, V_{z}\right)$ and the Sobel filters for each axis $(u, v, w)$ is used as the objective function. By minimizing the objective function, information on suitable $V_{x}, V_{y}$, and $V_{z}$ can be found. This equation is a rather direct method of calculating the equation 2.37 that is basically used in optical flow estimation.

The above formula is the most essential component of the proposed calculation method. However, we can predict several problems. First of all, since this method calculates the intensity of each voxel individually, this program is very sensitive to noise. In other words, the noise of each voxel can be recognized as a signal, and it results in overfitting. Next, in this method, if the moving distance of molecules or objects between two frames is too long, it becomes difficult to describe the subject. Calculation results tend to focus only on the initial and final positions without depicting a continuous movement. Conversely, if imaging is performed under sufficiently fast conditions, the moving distance of the fluorescence signal can be reduced. In addition, by including the derivatives of the velocity arrays $\left(\nabla V_{x}, \nabla V_{y}, \nabla V_{z}\right)$ in the error term, it is possible to prevent the local values from rapidly increasing or decreasing. Using this method, in order to make the smooth result and prevent overfitting, the objective function can be changed as follows.

$$
\Phi=\left|\Delta I-V_{x} \otimes u-V_{y} \otimes v-V_{z} \otimes w\right|+\lambda\left(\Sigma\left|\nabla V_{x}\right|+\Sigma\left|\nabla V_{y}\right|+\Sigma\left|\nabla V_{z}\right|\right)
$$

This is because the added error term tends to make the whole image smooth by preventing 
the sudden change in values. This penalized regression method is effective as a way to solve the overfitting problem that may occur in this program.

\subsection{Results}

\subsubsection{Intensity Profile of Standard Samples}

An appropriate brightness correction method to reconstruct a 3D image was selected among the three methods described before. First, the relative brightness of each image plane in a multi-plane setup data was compared using three calibration methods. The first one is a calibration method using fluorescent beads, and the second one is a method using a halogen lamp. The last one is the fitting result of the second method with an equation considering the prism structure. Since it is possible to correct the brightness of every image plane based on a single reference image plane, I compared the relative brightness of every image plane, after fixing the brightness of the first image plane equally. 


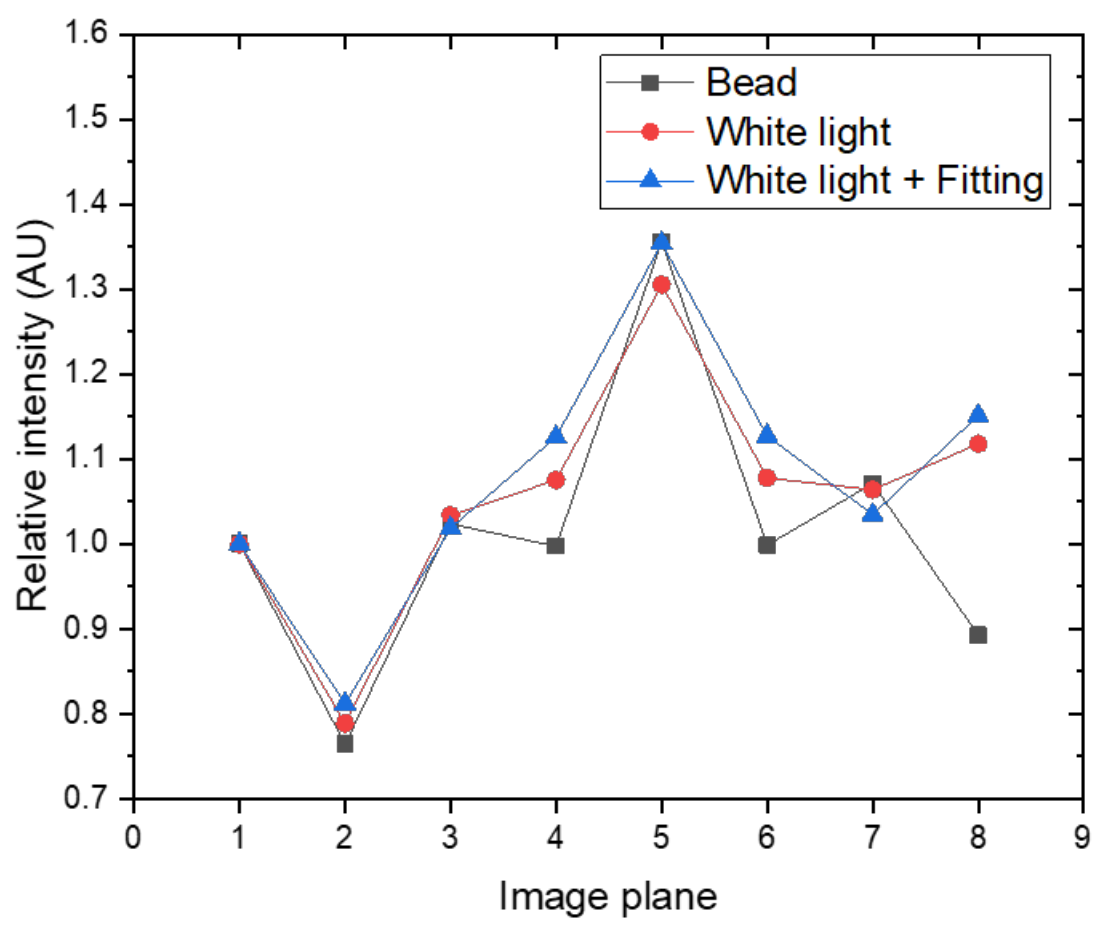

Figure 3.5: Intensity calibration results measured in different ways. The black line is the calibration result using the existing method, and the red line is the calibration result using the white light. The blue line results from fitting the result of the method using white light with the model function. When using the new methods, the intensity of the even-numbered image plane gradually increases.

From the comparison result, see figure 3.5, we can check which image plane has a large error in the brightness value. The brightness of the image plane corresponding to the odd number in the graph did not have a large difference according to the calibration method.(maximum 5\%) Oppositely, the brightness of the image plane corresponding to an even number increased by about $10 \%$ compared to the bead data when white light was used, and the brightness increased by about $15 \%$ in the fitting result using the model function. Due to the structural characteristics of the prism, the number of reflections and transmissions by the beam splitter is the same in odd-numbered planes except for the fifth image plane. The image planes having big intensity differences have different numbers of interactions with a beam splitter. From this tendency, we can infer that the cause of the brightness error is in the beam splitter (see table 3.1). 


\begin{tabular}{cllll}
\hline Plane & $\begin{array}{l}\text { Number } \\
\text { of } \\
\text { Reflection }\end{array}$ & $\begin{array}{l}\text { Number } \\
\text { of } \\
\text { Transmission }\end{array}$ & $\begin{array}{l}\text { Number } \\
\text { of } \\
\text { Reflection } \\
\text { (inter-laced } \\
\text { configuration) }\end{array}$ & $\begin{array}{l}\text { Number } \\
\text { of } \\
\text { Transmission } \\
\text { (inter-laced } \\
\text { configuration) }\end{array}$ \\
\hline 1 & 2 & 1 & 2 & 1 \\
2 & 2 & 1 & 3 & 0 \\
3 & 0 & 3 & 2 & 1 \\
4 & 2 & 1 & 1 & 2 \\
5 & 3 & 0 & 0 & 3 \\
6 & 1 & 2 & 1 & 2 \\
7 & 1 & 2 & 2 & 1 \\
8 & 1 & 2 & 1 & 2 \\
\hline
\end{tabular}

Table 3.1: Number of reflection, $n_{R}$ and transmission, $n_{T}$ inside the multi-plane prism. By adjusting the position of the camera, the inter-plane distance can be cut in half. This condition is called inter-laced configuration.

The model function to correct the brightness contains error values of the beam splitter as a major variable. Therefore, from the fitting result, we can quantify the error of splitting intensity ratio, and since the error range of a general beam splitter is known, we can determine if the calculation results are suitable. In the measurement data using halogen lamp, the brightness ratio between reflected light and transmitted light was about 46:54. This value is similar to the error range found in a typical single-beam splitter prism. Since the difference in reflectance of a prism depends on the wavelength, as described in 3.1.2, the intensity difference was measured at a limited wavelength range. By using the same optical filter to be used for fluorescence imaging of cells, and limiting the wavelength range, the error caused by the difference in wavelength was minimized.

In order to compare the accuracy of the three calibration methods, the a multi-plane setup data of a standard sample was reconstructed in 3D. The sample is the ArgoSIM pattern 'G' (ArgoLight) and has a structure of three crossing rings. The object has bigger volume than the field of view (FOV), and since it has a continuous structure that penetrates the entire FOV, we can evaluate continuity in $3 \mathrm{D}$ rendered images. The ring radius of the sample is $20 \mu \mathrm{m}$. However, using a 20x objective for the setup, the maximum height of FOV in multi-plane setup data is around $11 \mu \mathrm{m}$. Therefore, only $\frac{1}{4}$ of sample can be imaged. The captured FOV is the center of the sample where the rings intersect. The top and bottom of the sphere are outside the measurable volume. Due to the characteristics of wide-field illumination, light outside the image volume cannot be blocked. As a result, 
the boundary image planes are relatively brighter than the center.

In order to determine whether the intensity ratio between the image planes is appropriate, the maximum projection images on YZ plane was compared. First, in the fluorescent bead-based correction result, the brightness between each image plane repeatedly increased and decreased (see figure 3.6). This discontinuity indicates that the intensity ratio contains error, and the excessive increase in brightness is noticeable in the even numbered image plane. Next, in the result using white light and theoretical correction, the difference in brightness between adjacent image planes decreased. The fifth image plane showed a slight difference, but the difference was reduced compared to the calibration data using fluorescent beads. Therefore, the results of the newly presented correction method are more accurate. This result removes the effect of photobleaching, and the correction using the equation 3.4 works well.

Calibration with the new method also has a small error in the fifth image plane. This error can be corrected more by maximizing the continuity between adjacent image planes. Each image plane is in a continuous space and adjacent image planes must be similar to each other. In particular, there is more severe interference between the image planes because wide-field microscopy images lack optical sectioning capability. This characteristic of wide-field illumination has the effect of blurring the image, but also brings the continuity of vertical brightness. An objective function, $\Phi$ was set to minimize the difference in brightness between adjacent image planes like follows:

$$
\Phi=\sum_{i=2}^{7}\left|\lambda_{i-1} \cdot \lambda_{i} \cdot I_{i-1} \cdot I_{n}-\lambda_{i} \cdot \lambda_{i+1} \cdot I_{i} \cdot I_{i+1}\right|
$$

$I_{n}$ is the n-th image plane data. When the objective function, $\Phi$ is minimized, the weight factors, $\lambda$ s are optimized. When the image of the sample is corrected based on these weight factors, the result accurately represents the shape of the standard sample as shown in the figures 3.6 and 3.7. 

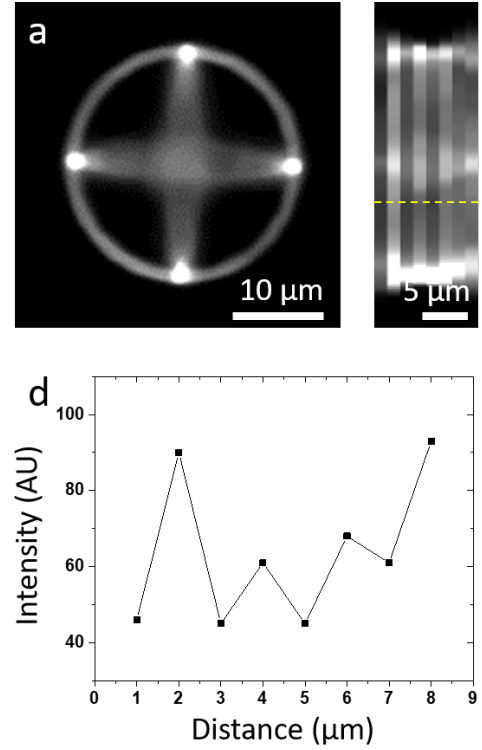
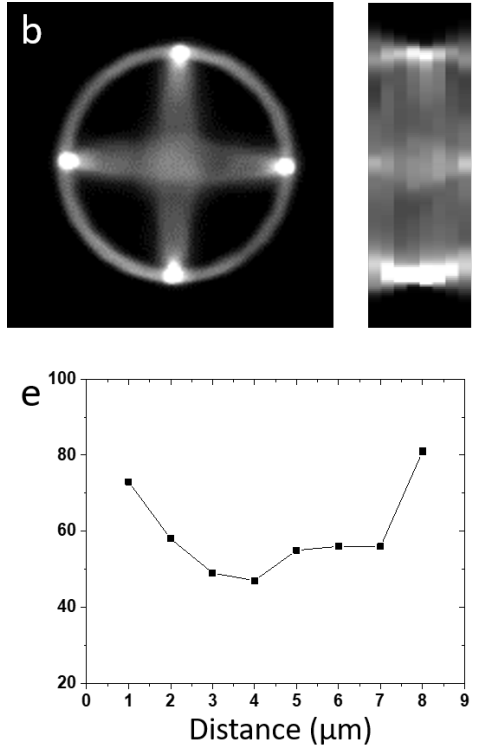
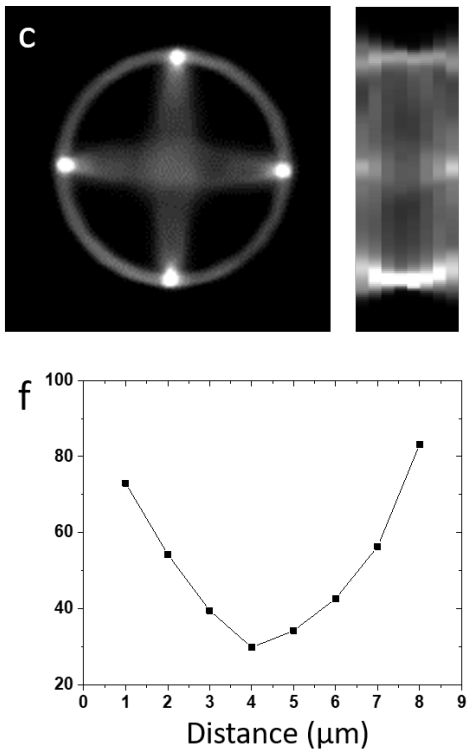

Figure 3.6: Comparison of calibration results. A standard sample of bead-like three rings was imaged. (a) I corrected the intensity of every image plane with the result of calibration using fluorescent beads. (b) Same image was corrected using the calibration result of white light and fitting. (c) A new function considering the continuous intensity in image volume was used to correct the intensity ratio. As a result of the white light and fitting method, the curve in the crosssection is reduced. As the result of the new function, the result describes the continuous shape of the sample.
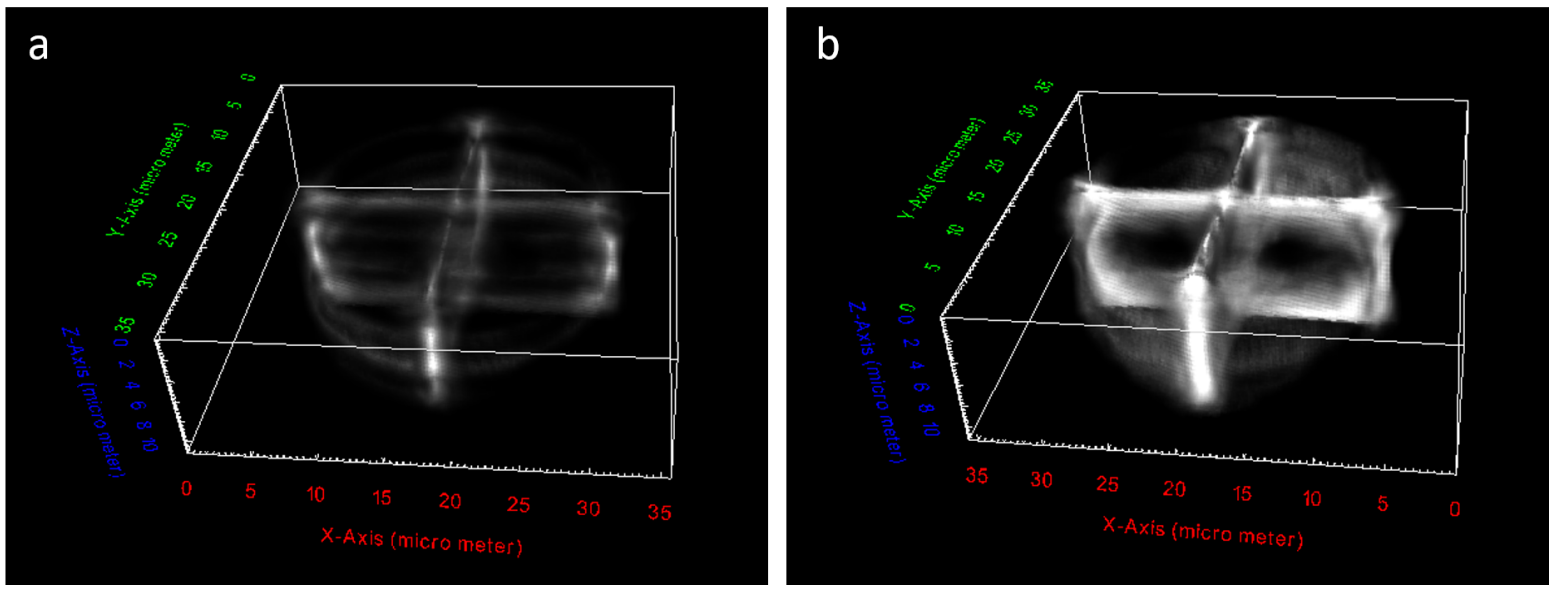

Figure 3.7: 3D rendering results of two different calibration methods. (a) Intensity was calibrated with the weighting factors resulted from the fluorescent bead measurement.(b) $3 \mathrm{D}$ rendering result after intensity correction using the new method of white light and fitting.

Accurate adjustment of the inter-plane intensity ratio can increase the accuracy and 
precision of deconvolution and optical flow estimation. If the intensity calibration parameter contains error, the intensity ratio between each image plane becomes irregular. The irregular intensity distribution may result in the contracted or distorted 3D image shape. (see figure 3.7). Therefore, accurate intensity calibration will be the best way to increase the reliability of this study result.

\subsubsection{Deconvolution Results - 3D-SUPPOSe}

With the intensity calibration method described in 3.1.2, a three-dimensional image without distortion is constructed. As a next step, the performance of the program to solve the missing cone problem and to sharpen the image was verified. Before directly analyzing the data of the multi-plane setup, in order to verify the performance of this program, the deconvolution results of 3D-SUPPOSe were compared with the other deconvolution methods. The deconvolution results of ThreeDeconv and Lucy-Richardson algorithm were used for the comparison. Using the standard sample image in the paper about ThreeDeconv[59], the characteristics and limitations of the three deconvolution programs were compared.

Using the method described in 3.1.2, a 3D image without distortion of brightness can be constructed. The next step was to verify the program's performance to sharpen the image of the multi-plane setup. In order to verify this program's performance, I directly compared the 3D-SUPPOSe result with previously described deconvolution program results for $3 \mathrm{D}$ wide-field microscopes. The image was deconvolved using ThreeDeconv and the Lucy-Richardson algorithm, and I compared the 3D-SUPPOSe deconvolution results with the other deconvolution results considering precision and artifacts.

The standard sample is a hollow bead with a diameter of $6 \mu \mathrm{m}$, and the bead has a fluorescent shell of $1 \mu \mathrm{m}$. In the paper about ThreeDeconv,[59] the deconvolution result was directly compared with the other results using various deconvolution programs, and the standard image was used to explain the characteristics of ThreeDeconv. For the deconvolution of this image, I used the same PSF for the three deconvolution methods. The iteration number of the Lucy-Richardson algorithm was fixed as 200 . This is a large enough number to get clear images.[94] 


\begin{tabular}{ccccc}
\hline & Ground-truth & 3D-SUPPOSe & $\begin{array}{l}\text { Lucy- } \\
\text { Richardson }\end{array}$ & ThreeDeconv \\
\hline Size (lateral) & $6.0 \mu \mathrm{m}$ & $5.8 \mu \mathrm{m}$ & $6.0 \mu \mathrm{m}$ & $5.9 \mu \mathrm{m}$ \\
Shell (lateral) & $1.0 \mu \mathrm{m}$ & $0.9 \mu \mathrm{m}$ & $0.9 \mu \mathrm{m}$ & $0.9 \mu \mathrm{m}$ \\
$I_{\text {shell }} / I_{\text {core }}$ (lateral) & $\infty$ & 258.4 & 6.16 & 14.72 \\
Size (axial) & $6.0 \mu \mathrm{m}$ & $6.1 \mu \mathrm{m}$ & $6.0 \mu \mathrm{m}$ & $5.7 \mu \mathrm{m}$ \\
Shell (axial) & $1.0 \mu \mathrm{m}$ & $1.4 \mu \mathrm{m}$ & $1.7 \mu \mathrm{m}$ & $1.8 \mu \mathrm{m}$ \\
$I_{\text {shell }} / I_{\text {core }}$ (axial) & $\infty$ & 130.96 & 2.06 & 11.01 \\
\hline
\end{tabular}

Table 3.2: In order to compare the deconvolution results, the size, thickness, and intensity ratio of the bead were listed. The size and thickness were measured based on the positions having half maximum intensity.
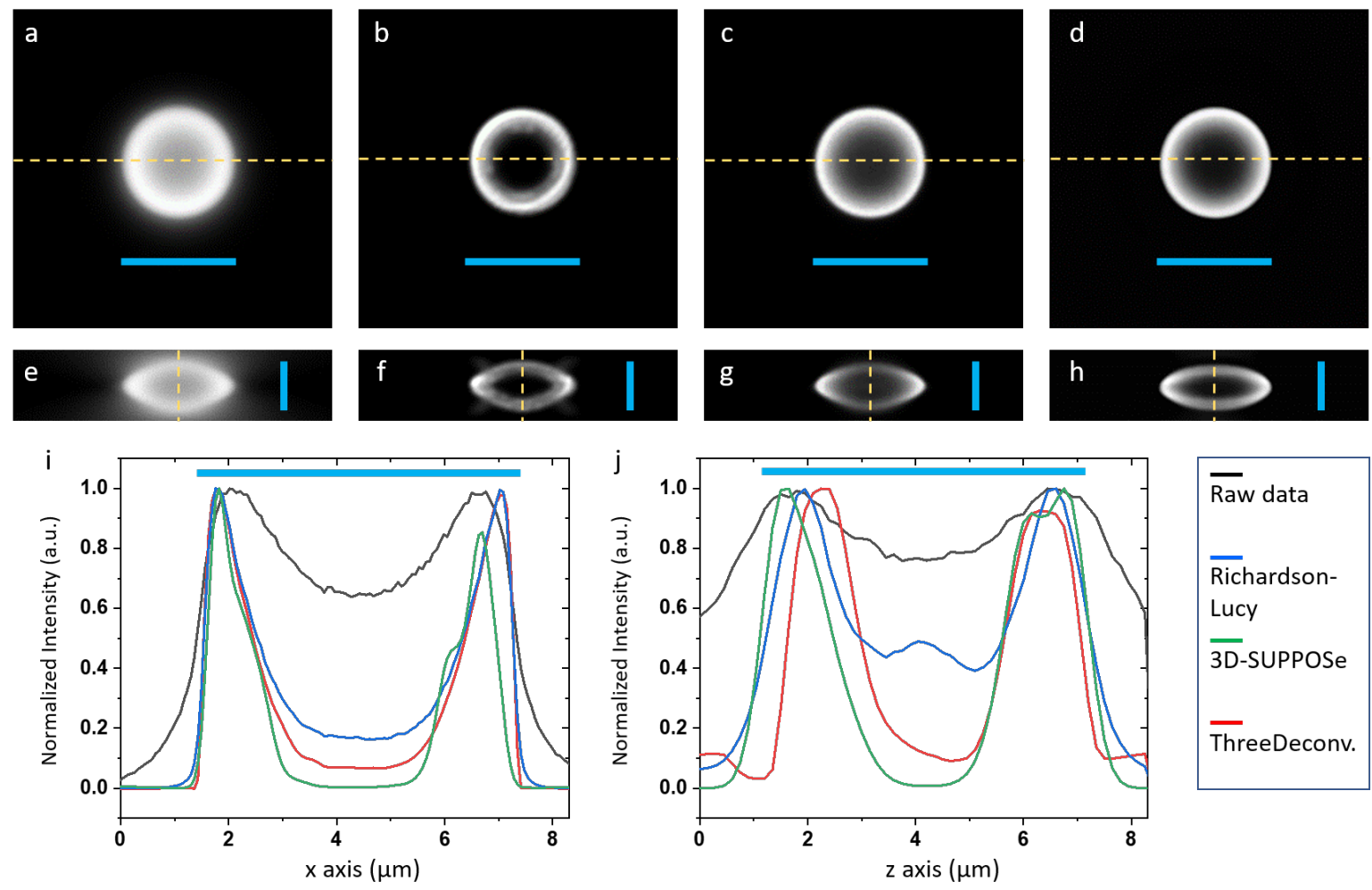

Figure 3.8: Comparison of $6 \mu \mathrm{m}$ bead images with different deconvolution methods. Transverse (xy plane) image intensity of a yellow-green fluorescent bead in (a) a widefield image, deconvolved with algorithms (b) 3D-SUPPOSe, (c) ThreeDeconv, and (d) Lucy-Richardson. Lateral cross section images (xz plane) are provided correspondingly in (e) to (h). (i) Cross-section intensity plot along the dashed yellow line in (a) to (d). (j) (i) Cross-section intensity plot along the dashed yellow line in (e) to (h).

As a result of the deconvolution, the image as shown in the figure 3.8 was obtained. For the evaluation of the results, the important points are contrast, bead size, and artifacts. 
In order to accurately compare the size of the bead, the diameter of the bead and the thickness of the fluorescence shell were compared. Bead sizes in the deconvolution results were measured in cross-sections for the xy and xz planes. For direct comparison, two cross-sections passing through the center along the $\mathrm{x}$-axis and $\mathrm{z}$-axis in each image are plotted together. In the cross-section along the x-axis of every deconvolution result, the shell of the bead was expressed as a similar thickness, $1 \mu \mathrm{m}$. The diameter along the $\mathrm{x}$-axis was also similar with the reference value of $6 \mu \mathrm{m}$. Although the cross section along the $\mathrm{z}$-axis shows similar diameter with $\mathrm{x}$-axis, the vertical diameter in ThreeDeconv result was reduced by about $300 \mathrm{~nm}$, less than $5 \%$ of the diameter.

To verify the second evaluation factor, contrast, the brightness difference between the core and the shell of the bead was compared. If the blurring effect is removed through deconvolution, the boundary between a dark area and a bright area should be clearer than before the convolution. Since the shell of the bead generates a fluorescence signal, but the core does not, the efficiency of deconvolution can be evaluated through the difference in brightness between the two regions. The three programs showed different trends in describing the brightness of the two regions. First, the Lucy-Richardson algorithm describes the shape smoothly, but the method was inferior in contrast. The Lucy-Richardson result has the highest intensity of the core region among the three programs, and the z-axis cross-section of the Lucy-Richardson result showed a higher core intensity than the outside of the core. Second, ThreeDeconv showed good contrast in all directions compared to Lucy-Richardson. The third one, 3D-SUPPOSe, resulted in the brightness of the core converging to 0 , but the boundary between the core and the shell was rough. This is a disadvantage that follows the calculation method using several discontinuous points. As suggested in the SUPPOSe paper[73], re-blurring with a Gaussian function of a small size may help to overcome the problem.

About artifacts, each method has different problems. In Lucy-Richardson, the center intensity of the bead was slightly increased. In ThreeDeconv, the vertical size was decreased less than $10 \%$ of the diameter. 3D-SUPPOSe result had rough surface, and very low intensity residue spreading out like PSF shape around the bead appears. However, the intensity enhancement of the central part shown in Lucy-Richardson is a big problem reaching $50 \%$ of the maximum intensity, but the error of ThreeDeconv is a small error of less than $10 \%$ compared to the size of the bead. The rough surface of 3D-SUPPOSe can be adjusted through additional treatment, re-blurring. 
Combining the three evaluations, although all three programs have different characteristics, they do not show a significant difference in performance as a deconvolution program of a 3D wide-field microscope. The Lucy-Richardson algorithm has the lowest computational cost, and ThreeDeconv is suitable for images with strong noise. 3D-SUPPOSe showed the clear shape of the object. All programs have their own merits, such as accuracy, low noise, and computational cost.

When evaluating performance as a general deconvolution program, it is sufficient to compare the three conditions mentioned above. However, as a deconvolution method of multi-plane setup images, we need to additionally consider the effect of interpolation. Multi-plane setup measures a limited number of two-dimensional images and reconstructs them into three-dimensional images. The axial size of the voxel is larger than the lateral size, and only eight limited voxels are positioned along the z-axis. Therefore, increasing the axial sampling frequency in order to accurately express the information in $3 \mathrm{D}$ space is a necessary process to reconstruct three-dimensional images from the multi-plane setup data. For this purpose, we need to increase the sampling rate by interpolation along the z-axis. Therefore, the most suitable deconvolution program for multi-plane setup data needs to have the least artifacts by interpolation.

In order to evaluate the interpolation effect on deconvolution, the two times interpolated result along the z-axis was deconvolved. After deconvolution of the interpolated bead image, the size, contrast, and artifact of the bead were evaluated from each deconvolution result. First, in 3D-SUPPOSe, the size was expressed very accurately. In both the XY plane and the XZ plane, the size of $6 \mu \mathrm{m}$ was accurately described, and the fluorescent shell was also clearly revealed. Second, in the case of Lucy-Richardson, the size of the bead in the XY plane was well described, but strong artifacts occurred at the boundary between the core and the shell. In the $\mathrm{XZ}$ plane, the vertical size was enlarged due to the additional blurring. Third, in the XY plane result of ThreeDeconv, the size appeared exactly with no significant difference from 3D-SUPPOSe. However, in the XZ plane, the vertical size reduction was stronger than before interpolation.

Second, contrast was compared from the results of the three programs. First of all, in the case of the Lucy-Richardson algorithm, the brightness inside the image was further strengthened, and the reversed intensity made it difficult to distinguish the shell from the core. Next, in the case of ThreeDeconv, the intensity of the core converges to 0 , showing high contrast. 3D-SUPPOSe also showed high contrast similar to ThreeDeconv. 
3.2. RESULTS

\begin{tabular}{ccccc}
\hline & Ground-truth & 3D-SUPPOSe & $\begin{array}{l}\text { Lucy- } \\
\text { Richardson }\end{array}$ & ThreeDeconv \\
\hline Size (lateral) & $6.0 \mu \mathrm{m}$ & $6.0 \mu \mathrm{m}$ & $6.0 \mu \mathrm{m}$ & $6.0 \mu \mathrm{m}$ \\
Shell (lateral) & $1.0 \mu \mathrm{m}$ & $0.9 \mu \mathrm{m}$ & $1.5 \mu \mathrm{m}$ & $0.9 \mu \mathrm{m}$ \\
$I_{\text {shell }} / I_{\text {core }}$ (lateral) & $\infty$ & 1000.00 & 0.69 & 1000.00 \\
Size (axial) & $6.0 \mu \mathrm{m}$ & $6.0 \mu \mathrm{m}$ & over $8.0 \mu \mathrm{m}$ & $5.7 \mu \mathrm{m}$ \\
Shell (axial) & $1.0 \mu \mathrm{m}$ & $1.0 \mu \mathrm{m}$ & N.A. & $1.2 \mu \mathrm{m}$ \\
$I_{\text {shell }} / I_{\text {core }}$ (axial) & $\infty$ & 1000.00 & 0.004 & 1000.00 \\
\hline
\end{tabular}

Table 3.3: In order to compare the deconvolution results after interpolation, the size, thickness, and intensity ratio of the bead were listed. The size and thickness were measured based on the positions having half maximum intensity.
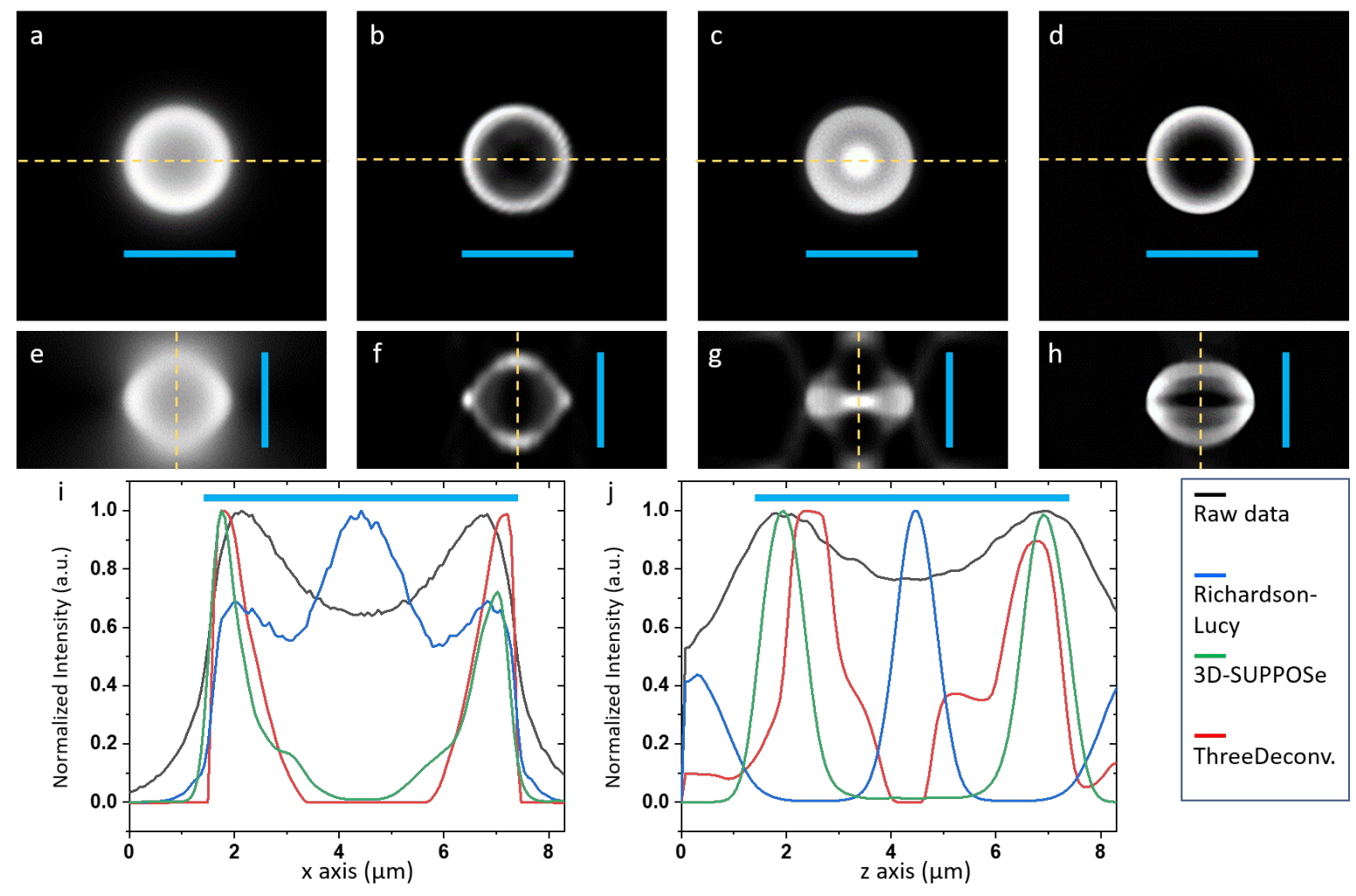

Figure 3.9: Comparison of $6 \mu \mathrm{m}$ bead images with different deconvolution methods after $2 \mathrm{x}$ interpolation along the z-axis. Transverse (xy plane) image intensity of a yellow-green fluorescent bead in (a) a wide-field image, deconvolved with algorithms (b) 3D-SUPPOSe, (c) ThreeDeconv, and (d) Lucy-Richardson. Lateral cross section images (xz plane) are provided correspondingly in (e) to (h). (i) Cross-section intensity plot along the dashed yellow line in (a) to (d). (j) (i) Cross-section intensity plot along the dashed yellow line in (e) to (h). 
Finally, comparing artifacts, Lucy-Richardson and ThreeDeconv confirmed that artifacts were strengthened after interpolation. According to Lucy-Richardson, the intensities of the bead core and the shell were reversed after interpolation. At the same time, the bead has a shape that converges to the plane. This artifact can be reduced by lowering the iteration number, but there is an additional problem that we cannot blur efficiently with a low iteration number. In ThreeDeconv, the vertical size was decreased, and additionally, a step-like intensity pattern was shown along the vertical direction. The artifacts shown in the two programs are the same problem with the artifacts of the image before deconvolution, but the effect after interpolation was getting more significant. On the other hand, 3D-SUPPOSe fully depicts the shape of the bead without enhancing the artifacts that appeared before.

\subsubsection{Combination of Multi-plane and 3D-SUPPOSe}

In the previous comparison, it was confirmed that 3D-SUPPOSe generates the best deconvolution result when we use the deconvolution methods with interpolation. Interpolation plays an important role in multi-plane image analysis. The axial sampling rate of the multi-plane setup is inferior to the lateral sampling rate. Since it only has eight limited image planes, it is necessary to increase the axial sampling rate for realistic 3D image rendering. By increasing the axial sampling rate using interpolation to make the spatial frequency the same in all directions, realistic 3D image rendering is possible. As the comparison results, 3D-SUPPOSe shows the best deconvolution results for an interpolated images. Therefore, in this study, a measurement method using a multi-plane setup combined with the 3D-SUPPOSe is used for live-cell imaging.

As described above, the multi-plane setup has structurally the low sampling rate along the axial direction. The number of image planes along the z-axis generated in the multiplane setup is only eight. Since the structure of the prism determines this number, it cannot be changed. The prism structure also fixes the inter-plane distance along with the number of image planes. The structure of the prism is designed to satisfy the superNyquist sampling rate [32] as the fixed interplane distance is sufficiently smaller than the vertical size of the measured PSF. Therefore, it is possible to precisely depict the shape of an object by appropriately increasing the axial sampling rate with interpolation method.

For the analysis of the measured ArgoSIM pattern 'I', the sampling rate along the z-axis 
was increased through interpolation. Due to the stair pattern of the sample, we can precisely compare the vertical positioning accuracy using the ArgoSIM pattern 'I'. Fourier interpolation was used to increase the space for z-axis spatial frequencies. Since the lateral frequency is about six times higher than axial sampling frequency, we performed six-fold interpolation along the axial direction. Interpolation was performed two more times to get higher sampling rate along the every direction. Finally, two-fold interpolation was performed along the xy-axis and twelve-fold interpolation was performed along the $\mathrm{z}$-axis direction. After deconvolution, the size of the voxel is $120 \times 120 \times 122 \mathrm{~nm}$, and the voxel has a shape close to a cube.

In the deconvolution result for an interpolated image using 3D-SUPPOSe, the image was efficiently cleared. The average height and width of each step are $477 \pm 53 \mathrm{~nm}$ and $623 \pm 168 \mathrm{~nm}$. Before deconvolution, the axial and lateral sizes were $1350 \pm 113 \mathrm{~nm}$ and $1492 \pm 417 \mathrm{~nm}$. The spatial frequency of the sample image was increased more than two times. Considering the limited z-axis spatial frequency of the multi-plane setup, it can be an excellent way to overcome the prism's structural limitation and the missing cone problem of wide-field illumination. 

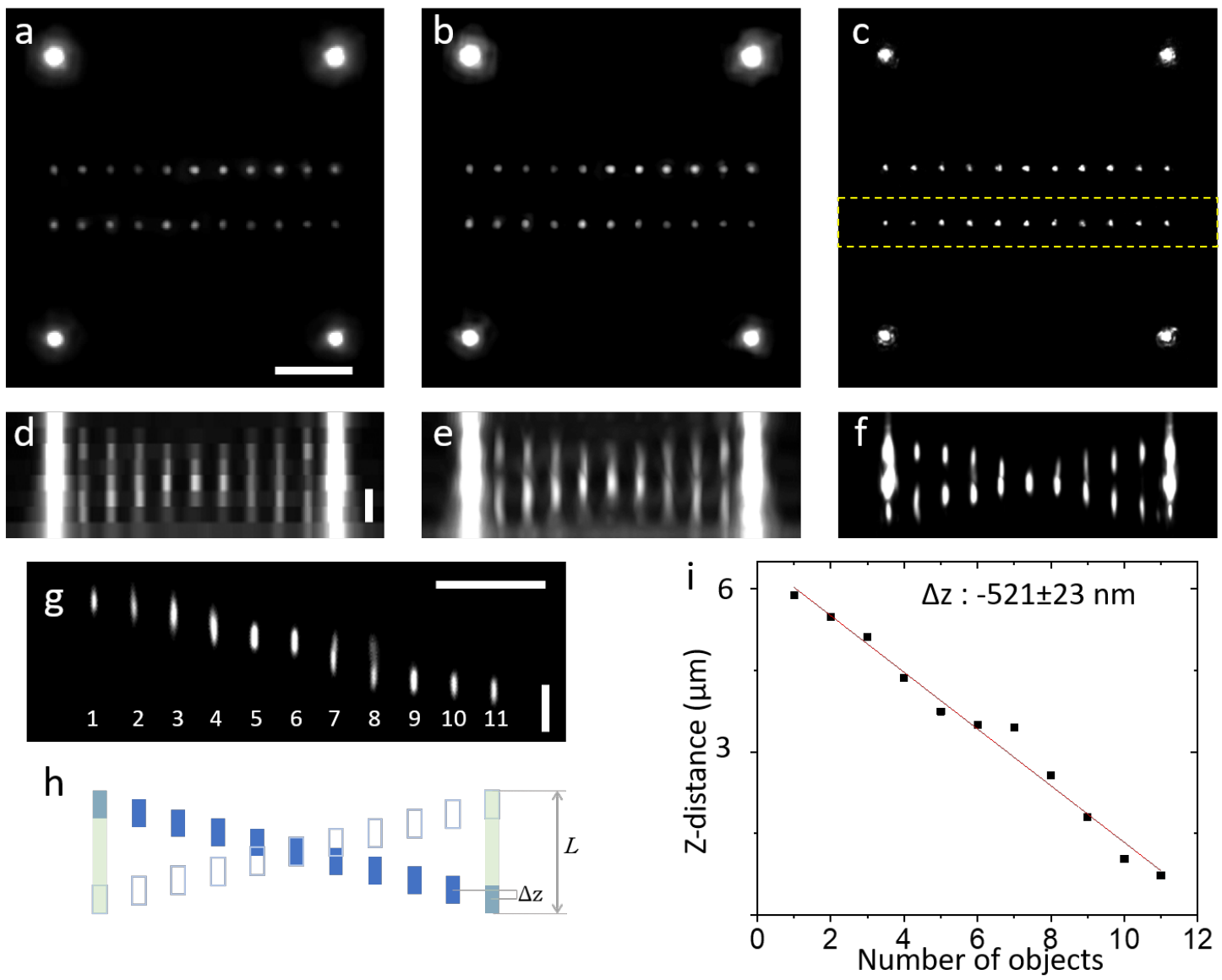

Figure 3.10: Resolution enhancement of an ArgoSIM pattern 'I', We imaged the ArgoSIM pattern 'I'. The pattern has the structure of crossing stairs like structure in (h). The distance between the stairs $(\Delta z)$ is $500 \mathrm{~nm}$, and the total height of the structure $(L)$ is $5 \mu \mathrm{m}$. The size of each step is less than $1 \mu \mathrm{m}$. The raw data (a,d) was interpolated (b, e), and deconvolved (c, f). We cropped the yellow dotted square part in c and plotted the lateral view of the cropped image $(\mathrm{g})$. The center of fluorescence signal of each spot was calculated, and we plotted the z position of each spot (i). The fitted slope of stairs is about $521 \pm 23 \mathrm{~nm}$. The size of scale bars is $10 \mu \mathrm{m}$ for $\mathrm{x}, \mathrm{y}$-axes and $3 \mu \mathrm{m}$ for z-axis.

The vertical position of each step indicated in the deconvolution results was measured by COFM calculation. In order to confirm the positional accuracy of the deconvolution multi-plane setup, the positions of each object were compared. Each step is arranged at regular intervals along the x-axis, and the height difference of each step is about 500 $\mathrm{nm}$. The position of each COFM was calculated, and the vertical position of each step was plotted. The slope was about $521 \pm 23 \mathrm{~nm}$, and the fitting error was about $5 \%$. The COFM values of each object showed a significant difference in the range of less than $15 \%$ from the fitting results. In particular, the height of objects 6,7 and 8 of figure $3.10 \mathrm{~g}$ has 
been particularly increased. The images of the objects are located on the fifth and sixth image planes, and the intensity ratio between the image planes have significant error. Due to the error of relative intensity, the positioning error of the three objects can be induced. An explanation of the exact cause will be possible after further experiments.

\subsubsection{D Bead Flow Analysis}

Using the calibration method and the 3D-SUPPOSe with interpolation, we can convert the multi-plane setup data into a precise three-dimensional image. By arranging the three-dimensional images according to time, we can make a four-dimensional fluorescence movie. The fluorescence movie includes information on how the three-dimensional distribution of fluorescent molecules changes over time. To describe the motion in live cells, the performance of the new optical flow estimation method described in 3.1.4 was verified. In order to verify the performance of this estimation method, the flow of a single bead was analyzed.

The flow of these fluorescent beads was measured to explain the mass transfer occurring at the droplet boundary. The measured image includes movement for several beads, and the algorithm was evaluated by analyzing an isolated bead having the most clear direction. When several beads are adjacent to each other, the motion of each bead can be influenced by each other. When a bead is isolated from the surrounding beads, the direction of the flowing bead follows purely the mass transfer, and the direction is simpler than others. Therefore, I selected a condition in which the signal of a single bead is as independent as possible from other beads. Only the area and time in which a single bead is clearly visible among the entire image were selected to analyze.

3D-SUPPOSe was used to reconstruct the three-dimensional bead image in the selected range. Before using 3D-SUPPOSe, the brightness was calibrated using the explained calibration method. After that, using Fourier interpolation, the number of data voxels in the selected image was increased by two times along the lateral direction and twelve times along the axial direction. Finally, the image was reconstructed into a threedimensional image with equivalent spatial frequency for all directions. Next, the image was deconvolved by using 3D-SUPPOSe.

The movement of the bead was analyzed in the precisely reconstructed 3D image using the methods. In order to evaluate the accuracy of the newly used optical flow estimation, the calculation results were compared with the COFM change over time. As shown in 
figure 3.11, the calculated COFM change was greatest along the $\mathrm{x}$-axis. Small movements were also found along the y-axis and z-axis. The direction of the flowing bead was almost constant throughout the whole movie, and the speed increased slightly. This speed increase seems to be occured as the bead approaches the boundary of the water drop.

In the new optical flow estimation result, velocity values of $11 \mathrm{x} 11 \mathrm{x} 11$ voxels $(2.6 \mu \mathrm{mx} .2 .6$ $\mu \mathrm{mx} 2.6 \mu \mathrm{m}$ ) corresponding to the entire bead size were averaged in order to compare the COFM with the estimated value of whole bead. The accuracy of the calculations was evaluated by comparing the cumulative velocity of the bead over time with the position of the COFM. As a result of comparison, the overall trend of movement shown by COFM was expressed similarly to the result of optical flow estimation. In particular, the similarity of the $\mathrm{x}$-axis information with large movement was high. However, the program considers all signal fluctuations as meaningful signals. Therefore, the y and z-axis information, which has a relatively small motion, did not accurately describe the direction compared to the $\mathrm{x}$-axis.
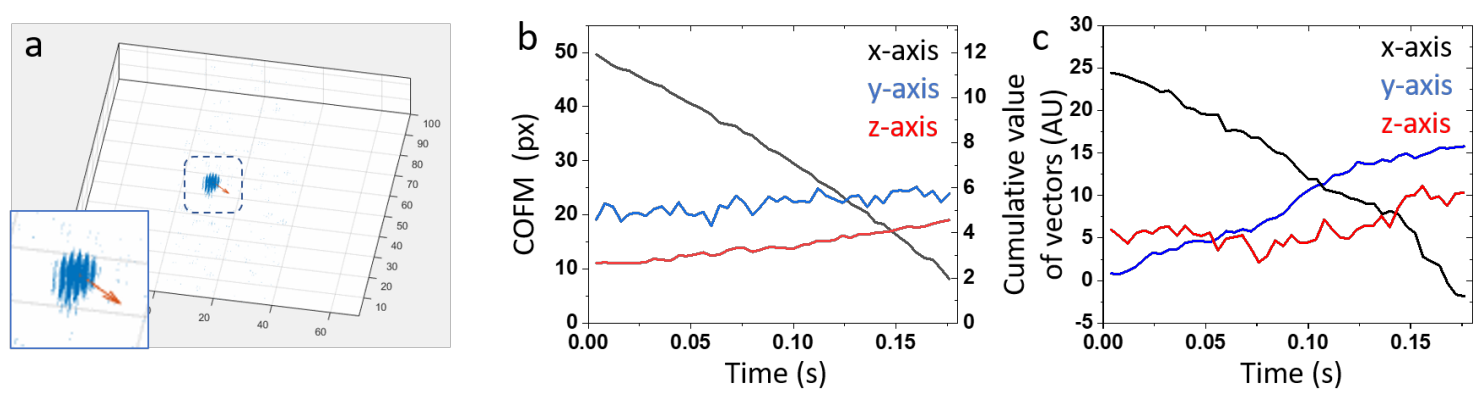

Figure 3.11: Flow estimation result. (a) 3D plot of bead in the image volume. The direction of estimated flow in the time frame is expressed as a red arrow. (b) COFM change. The black line is the positions on the $\mathrm{x}$-axis of COFM. The blue line is the positions on the $y$-axis, and the red line is the positions on the z-axis. (c) cumulative plot of three vectors. The color-code for each axis is the same as the COFM plot.

\subsection{Discussion}

The newly proposed optical flow estimation method expresses the flowing direction of the bead. However, it is still insufficient to describe small motion due to the influence of noise. This program was greatly affected by noise, so the results were not uniform at every moment. Therefore, in order to depict small movements, noise must be removed first. Programs to remove noise from images are being developed[72], and if these programs 
are applied properly, the denoising method is expected to help solving the problem of the flow estimation program.

However, the flow estimation method analyzes the movement with a large velocity well. This showed the possibility of optical flow estimation concept based on voxel level calculation. In previous studies on cell image analysis using optical flow estimation, the method of directly counting the signal size of voxel was not used.[13, 51, 71] By performing a direct operation on a voxel, the smallest unit of a three-dimensional image, we can directly calculate the molecular motion occurring in a narrow area.

3D-SUPPOSe also calculates the distribution of molecules at the single voxel level. This program was designed to estimate the ideal distribution of virtual molecules by directly comparing the distribution of virtual fluorescent molecules with the measured images. All fluorescence images depict quantitative positional information of the fluorophores. However, the accuracy tends to decrease due to the influence of noise and light diffraction. Image deconvolution removes the blur and clearly depict the distribution of fluorescent molecules. In particular, 3D-SUPPOSe was designed to localize the positions of virtual molecules and provide information on the most probable fluorescence distribution especially along the vertical axis. By this, it helps to overcome the 'missing cone' problem of wide-field microscopy.

3D-SUPPOSe, which depicts the ideal three-dimensional distribution of fluorescent molecules, is suitable for the analysis of multi-plane data. 3D-SUPPOSe showed better performance than other programs when we use this method with interpolation. Interpolation plays an important role in multi-plane data analysis. Since the data of the multi-plane setup has only eight limited image planes, the axial sampling rate is very low. In order to render realistic 3D images by increasing the insufficient sampling rate, interpolation along the z-axis is important. 3D-SUPPOSe shows the least artifacts after interpolation and can estimate the most precise structure. Therefore, it overcomes the disadvantages of multi-plane setup and allows the setup to be used for fast 3D fluorescence imaging.

Data analyzed by 3D-SUPPOSe and new optical flow estimation deliver quantitative information to us. For this purpose, brightness calibration of a multi-plane setup is a basic and essential task. These two analysis programs presuppose the accuracy of raw data. If the information in the raw data is incorrect, the accuracy of all analysis will be lost, and the error inside the raw data will be transmitted as the form of artifacts. 
Therefore, the accuracy of the brightness calibration is the basis for the accuracy of every analytical technique described above.

The newly proposed calibration method reduces the possible errors. There is an existing brightness calibration method, but it still contains possibility to include small experimental error like photobleaching issue. Reducing the potential errors improves data reliability. Therefore, fundamentally removing the effect of photobleaching and correcting the intensity using the theoretical model have an important meaning for stable calibration. The additional equation 3.2.1 for correcting the error included in the result allows us to obtain more accurate calibration data.

All of these methods were created with the purpose of expanding the usability of the multi-plane setup. A multi-plane setup structurally needs the brightness correction of each image plane. Also, since interpolation is an essential process for $3 \mathrm{D}$ image rendering with realistic aspect ratio, 3D-SUPPOSe, which shows the best performance after interpolation, is a suitable program for multi-plane setup image analysis. Finally, in order to derive quantitative information from the multi-plane setup data, a new optical flow estimation was designed. Using this method, it was possible to measure the flow of fluorescent molecules in a narrow range through the calculation of voxel units. Therefore, all techniques for reconstructing a three-dimensional image and obtaining quantitative information from a multi-plane setup data are introduced in this chapter.

\subsection{Summary}

- In order to accurately correct the intensity ratio between image planes of the multiplane setup, a new calibration method was developed. This method can reduce the experimental errors that occured during the conventional calibration method. It can reduce the possibility of an error with the equation calculated based on the prism structure and the measurement method using white light.

- I optimized SUPPOSe, a deconvolution method based on virtual emitters, for data analysis in a multi-plane setup. Gradient descent algorithm was used for precise estimation, and the program was restructured for efficient memory consumption.

- A new fluorescence flow estimation method was proposed to calculate the number 
of emitters moving in the image. 



\section{証 \\ Live Cell Imaging - CARDiomyocyte}

U sing 3D-SUPPOSe and a multi-plane setup, the three-dimensional movement of cardiomyocytes, the major cells constituting cardiac muscle tissue, was observed. These cells induce the cardiac motion of repeated contraction and relaxation with $12 \mathrm{~Hz}$ speed. As the function of the heart is important in maintaining human life, research on cardiomyocytes is also active. Therefore, although the mechanism for the cardiomyocytes movement is well studied, still, studies of some organelles and molecules are not enough. In this chapter, we will focus on the three-dimensional motion of the z-discs, which is one of the main structures of cardiomyocytes and is known as a relatively static structure.

Cardiomyocytes are composed of Z, M, I and A bands. [54] The A band is composed of myosin and actin filaments. The interaction between myosin and actin filaments induces cell contraction and relaxation according to the flow of calcium. Z-bands, called Z-discs, support actin filaments during contraction and relaxation. Unlike the A-band that directly drives cardiomyocyte movement, studies on the Z-disc are lacking. Cryo EM images of Z-disc structures were recently reported. However, research on dynamic structures of molecules is still in progress. 


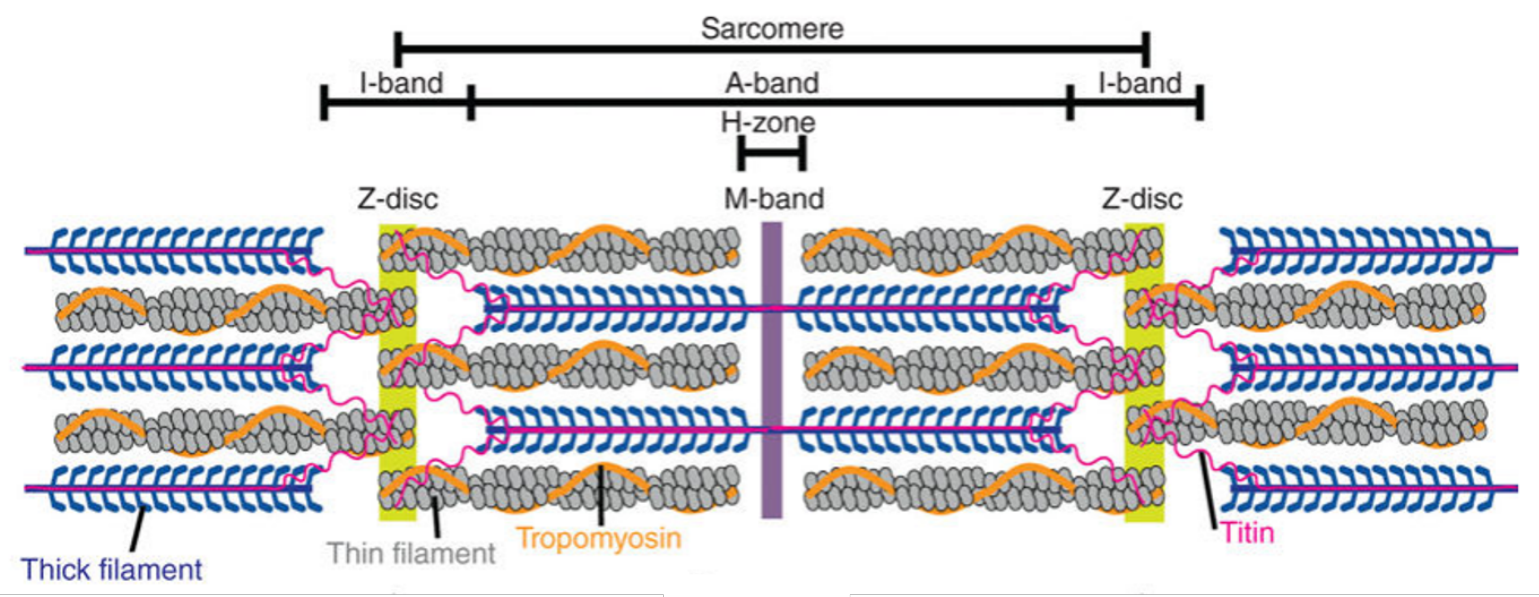

Figure 4.1: Schematic diagram of a cardiomyocyte showing three main filament systems: actin-based thin filament (grey), myosin-based thick filament (blue), and titin (pink). The lateral border of the sarcomere is the Z-disc. The I-band surrounds the Z-disc and is an area where thin filaments are not superimposed by thick filaments. The A-band consists of thin and thick filaments. The $\mathrm{M}$ band belongs to the $\mathrm{H}$ region where thick filaments do not interdigitate with thick filaments.[54]

The Z-disc is composed of several proteins, such as the muscle LIM protein, desmin, cypher, and $\alpha$-actinine-2 (ACTN-2). [95] Among them, ACTN-2 is known to directly connect the Z-disc and actin filaments. Since it is stably distributed in the Z-disc without two-dimensional movement, it is well known as a fluorescent labeling site than the function of the protein itself. Ribeiro et al. published a theory in 2014 regarding ACTN-2 regulation and interactions with proteins located around ACTN-2.[90]

In this chapter, I will analyze the movement of ACTN-2 in live cardiomyocytes to provide information on the function of ACTN-2. Since it has been used as a labeling site in various cardiomyocyte imaging studies, a method for attaching a fluorophore and tracking its location is already well studied. However, the fluorescence images were taken only in two-dimensional, and the high-resolution three-dimensional motion is still unknown. Therefore, here I analyze the motion of ACTN-2 using 3D-SUPPOSe multi-plane setup and discuss its meaning. 


\subsection{Method}

\subsubsection{Sample Preparation}

\section{Cardiomyocyte Culture}

The cells were provided with a micro-patterning dish from the Zimmermann group of the University Medical Center Göttingen. Human induced pluripotent stem cells (hiPSCs) were differentiated into hiPSC-derived cardiomyocytes and metabolically purified by glucose deprivation and lactate selection as described in ref [104]. Human iPSC-derived cardiomyocytes were cultured in Base Medium (RPMI 1640 with GlutaMAX supplemented with $2 \%$ B27 (Thermo Fisher Scientific), $1 \mathrm{mM} / \mathrm{L}$ sodium pyruvate (Gibco), $100 \mathrm{U} / \mathrm{mL}$ Penicillin, $100 \mu \mathrm{g} / \mathrm{mL}$ Streptomycin (Gibco) and $200 \mu \mathrm{M} / \mathrm{L}$ Ascorbic Acid (Sigma Aldrich)). Cells were detached with $20 \mu \mathrm{g} / \mu \mathrm{L}$ DNaseI (Calbiochem) and 0.025\% Trypsin (Gibco) in StemPro Accutase Cell Dissociation Reagent (Thermo Fisher Scientific) for $30 \mathrm{~min}$ at room temperature. Cells were resuspended in Base Medium with $5 \mu \mathrm{M} / \mathrm{L}$ Rock inhibitor Y-27632 (Stemgent) and 250 thousand cells were plated on micro-patterned imaging dishes. Medium was replaced the next day with Base Medium and exchanged every other day until further analysis. Citrine was fused to ACTN-2 by genetic engineering.

\section{Image Dish Micro-patterning}

Imaging dishes were micro-patterned with Synthemax II-SC substrate (Corning Cat. No. $3535)$ in $60 \times 10 \mu \mathrm{m}$ rectangular shapes with an interval of $20 \mu \mathrm{m}$ using PDMS stamps either with or without an extra layer of $10-\mathrm{kPa}$ polyacrylamide substrates (unless noted otherwise) following the protocols of Camelliti et al.[22] and Ribeiro et al.[89].

\subsubsection{Imaging and image processing}

\section{Microscope Setup}

In the illumination path, a cyan laser $(473 \mathrm{~nm}, \mathrm{CNI})$ light is spatially filtered, expanded and collimated. Illumination was done by focusing the collimated light into the back focal plane of a 20x air objective (UPLFLN20X, 0.5 NA, Olympus). Fluorescence emission was collected by the objective the back-reflection and then transmitted through a Quadband beam splitter (Quad Line Beamsplitter R405/488/561/635 lambda/5, Semrock). A band-pass filter (520/45 nm, Semrock) was used for further filtering of the emission. 
In the detection path, a multi-plane prism is incorporated which enables to detect eight distinct but equally-spaced image planes along the optical axis (the depth of sample) [32]. These image planes exit the prism as two sets of four adjacent images. Two sCMOS cameras (ORCA-Flash 4.0 V2, Hamamatsu) record the image planes synchronously. A further lateral magnification factor of 1.33 is achieved using a telescope system comprised of two lenses with focal lengths of $150 \mathrm{~mm}$ and $200 \mathrm{~mm}$. A rectangular field stop is positioned at the focal plane of the microscope tube lens to adjust the field of view and to prevent any cross-talk between neighboring images on the cameras. A custom-built plexiglass enclosure was assembled around the microscope body as an incubator chamber. To control the temperature, oxygen, and carbon-dioxide level inside the chamber, a heater (AIRTHERM-SAT-1W, WPI) together with an environmental control unit (ECU-HOC, WPI) is exploited.

\section{Image acquisition and processing}

All movies were recorded at $20 \mathrm{~Hz}$ frame rate. After measurements, intensity correction, z-stack registration, noise removal, and Fourier interpolation were performed to create a sharp 3D image stack. Images were interpolated two times along $\mathrm{x}$, and $\mathrm{y}$-axes and twelve times along z-axis. The voxel size after interpolation was about $120 \mathrm{~nm}$ for each axis. For precise alignment at sub-pixel resolution, the StackReg[103] program was used. Maximizing the SNR is a critical issue for fast imaging. Increasing the speed leads to the problem of reducing the amount of signal. ACsN, a new noise filter for sCMOS cameras, has been reported. [72] ACsN was applied to maximize SNR. SNR was increased from $2.50 \pm 0.03$ to $3.9 \pm 0.13$ after denoising. The image stack was interpolated with Fourier interpolation Matlab code used for Fourier SOFI data analysis. [101] Finally, the image was deconvolved using 3D-SUPPOSe.

\subsection{Results}

\subsubsection{Planar Beating Motion of Single Live Cardiomyocyte}

Cardiomyocytes were placed on specially patterned plates to maintain polarity. Polarized cells retain their rod shape, and we induce cell polarization using the patterned plates so that we can clearly see the contractile movement. In order to stably maintain the vitality of cells, we imaged the shape while maintaining the $\mathrm{CO}_{2}$ level at about $37^{\circ} \mathrm{C}$. No electric device was used to induce the beating motion. 
Since sufficiently mature cardiomyocytes have beating motion like normal hearts, the maturity level was checked based on the beating cycle of the cells. The movement measured by the fluorescence signal of citrine attached to ACTN-2 showed a repetitive pattern with a period of about $1.75 \mathrm{sec}$. Although the cell movement was slow, it was within the normal range of $12 \mathrm{~Hz}$ of cardiomyocytes. The amplitude of the repeated motion was almost constant in all periods. Cells showed strong beating for a short period of time, within about $30 \%$ of the entire cycle, and there was no motion for the rest of the time. The contractions occurred for about $0.5 \mathrm{~s}$ in a single beating cycle. The cell contracted and relaxed rapidly, and it was motionless at other times in a single cycle. This motion follows the trends of already known healthy cardiomyocyte beating. [70]. Therefore, it can be considered as the beating motion of sufficiently mature normal cardiomyocytes.
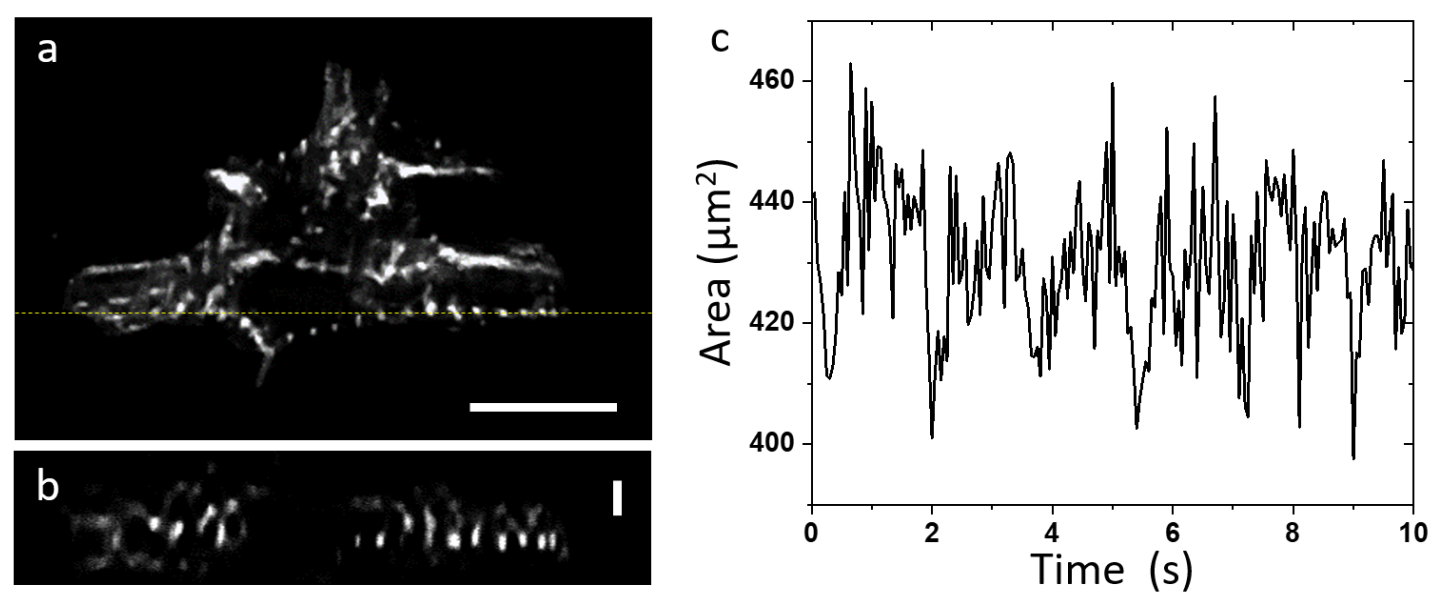

Figure 4.2: Figure a and $\mathrm{b}$ are the first frame of whole movie. $\mathrm{a}$ is the mean projection image on z-axis. $b$ is the cross section of the yellow dashed line. The size of the scale bar is $10 \mu \mathrm{m}$ in a, and $3 \mu \mathrm{m}$ in $\mathrm{b}$. Graph $\mathrm{c}$ shows area change of cardiomyocite over time.

The direction of movement was clearly seen in the 2D image.(Fig.4.3) The area change over time was about $5 \%$ of maximum area of z-projection image. Comparing the positional change of the z-projection image, the contraction and expansion occur periodically. However, when comparing the distribution of ACTN-2 in the 3D image, the pattern of vertical movement was not clearly visible. 

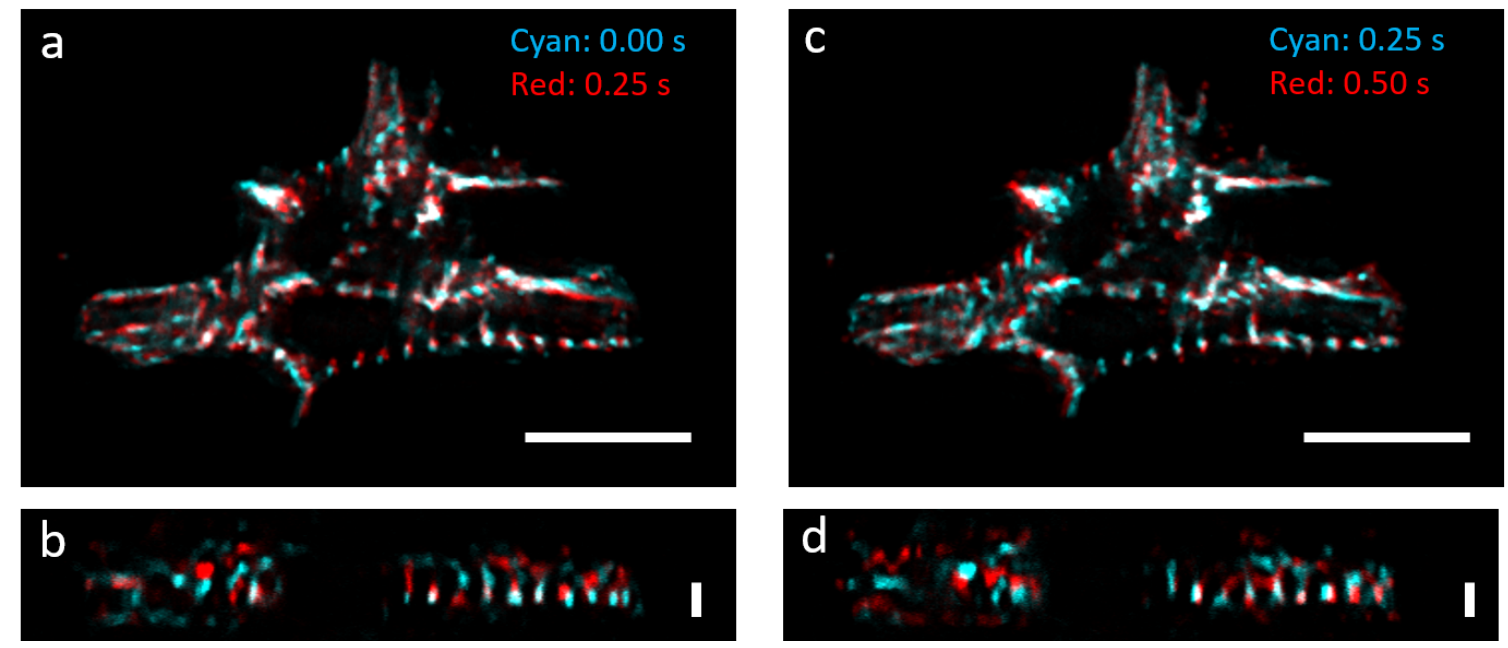

Figure 4.3: Images of cardiomyocyte (3D-SUPPOSe deconvolved multi-plane images of cardiomyocyte live cells).Figures $a, b, c, d$ are the overlapped images. In a and b, the first frame and sixth frame are overlapped. The cyan image is the first frame and red image is the sixth frame. The white area in the image is the region where two images overlap. In e and $f$, the sixth frame and eleventh frame were overlapped. The size of the scale bar is $10 \mu \mathrm{m}$ in a, c, and $3 \mu \mathrm{m}$ in $\mathrm{b}, \mathrm{d}$.

The direction of the beating motion was clearly visible in the two-dimensional movie. (see figure 4.3) To measure the area change, a threshold intensity was set as a half of the maximum brightness, and the area having higher intensity than the threshold value was measured. The maximum area difference during the whole cycle was about $5 \%$ of the maximum area. The periodicity can be confirmed through the area change of the z-projection image (see figure $4.2 \mathrm{~b}$ ). However, when comparing the distribution of ACTN-2 in 3D images, the vertical distribution was not uniform at every moment (see figure 4.3).

In order to understand the difference between the two- and three-dimensional spatial ACTN-2 distribution, the distribution change over time was analyzed by comparing image covariance. The similarity to the mean of the full-frame images was quantified using the normalized covariance as shown in the following equation.

$$
\operatorname{Cov}=\frac{\sum_{i=1}^{m} I_{n}\left(x_{i}\right) \cdot \bar{I}\left(x_{i}\right)}{\sqrt{\sum_{i=1}^{m} I^{2}\left(x_{i}\right)} \cdot \sqrt{\sum_{i=1}^{m} \bar{I}^{2}\left(x_{i}\right)}}
$$

$m$ is the number of voxels, $I_{n}$ is the nth image frame in the time series, and $\bar{I}$ is the 
arithmetic mean of all image frames in the series. The covariance of the z-projection shows a pattern that decreases periodically and returns. This graph of two-dimensional movie clearly shows the periods when the cardiomyocyte has strong motion and is stable without any motion. However, the covariance of the three-dimensional image stack was smaller than the covariance of the two-dimensional image, and the graph was similar to the two-dimensional graph but tended to be more complex.

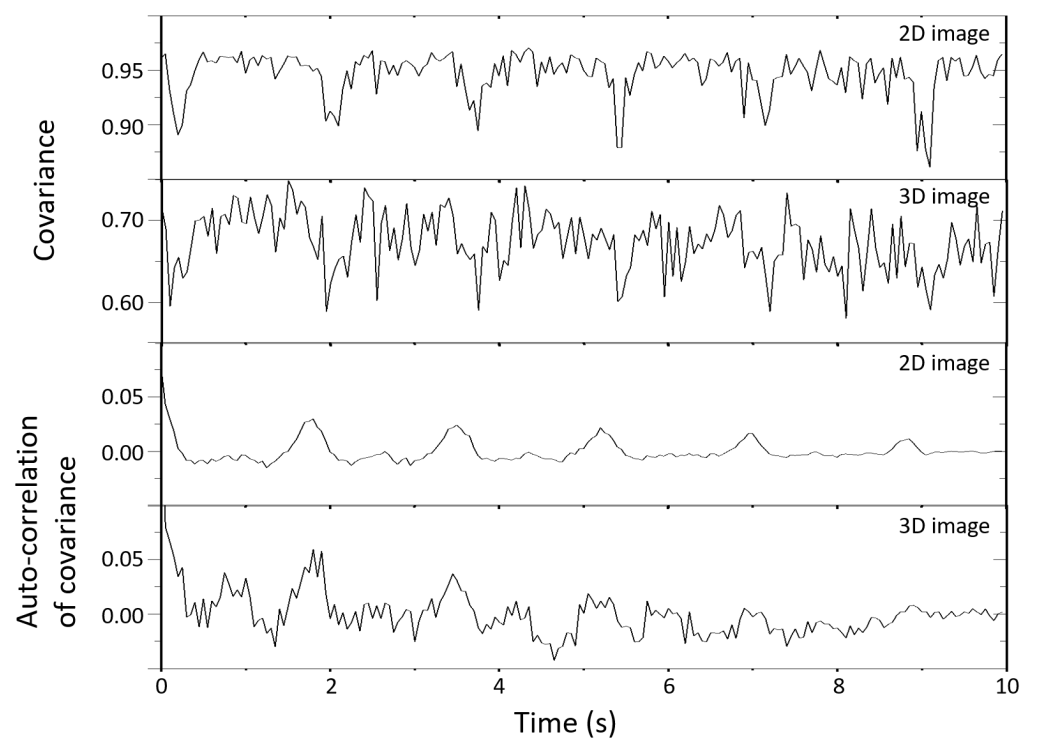

Figure 4.4: From the images in Fig. 4.3, we are able to compare how much the cell can move. The graphs in $\mathrm{g}$ are about the periodic beating motion. The two graphs on top are about the normalized covariance value with mean intensity image. We generated the mean intensity image from averaging the whole stack of $2 \mathrm{D}$ and $3 \mathrm{D}$ movie. In $2 \mathrm{D}$ motion, the periodicity of motion is clear, but the graph of $3 \mathrm{D}$ motion is more complex than $2 \mathrm{D}$ motion.

Several components with different frequencies were clearly identified through the autocorrelation of the two results. The autocorrelation graph for the two-dimensional covariance showed the beating frequency of normal cells. There is a peak close to 1.75 $\mathrm{Hz}$ in the autocorrelation of the 3D image covariance, and the graph is more complicated than the autocorrelation of the 2D image covariance. In particular, an increase in the signal was evident near the frequency of $1 \mathrm{~Hz}$. Based on this, it was confirmed that the three-dimensional ACTN-2 motion includes various components. 


\subsubsection{D Movement of $\alpha$-ACTN-2 in Single Z-discs}

The discordancy between two-dimensional and three-dimensional data suggests that ACTN-2 molecules may have vertical motion. To determine the exact cause, the threedimensional movement of each single Z-disc was analyzed. It is difficult to isolate the fluorescence signal of a single Z-disc from densely distributed Z-discs. On the other hand, the Z-disc isolated from the others has an independent motion from the adjacent Z-disc, and the separation of the fluorescence signal is also easy. Therefore, the region in which the least number of Z-discs are located was selected to be analyzed.

As a first step, the position of each single Z-disc was projected on the XY and YZ planes. As a result of the projection, repetitive movements were observed in the XY plane image, and fast and irregular motions were observed in the YZ plane image. The motion in the $\mathrm{XY}$ plane image repeated contraction and relaxation along the direction of the cell nucleus. On the other hand, the YZ plane image showed fast and irregular motion. When I averaged all YZ plane images, I found two vertically separated regions. When comparing each frame without averaging, bright spots were predominantly distributed in one of the two locations.
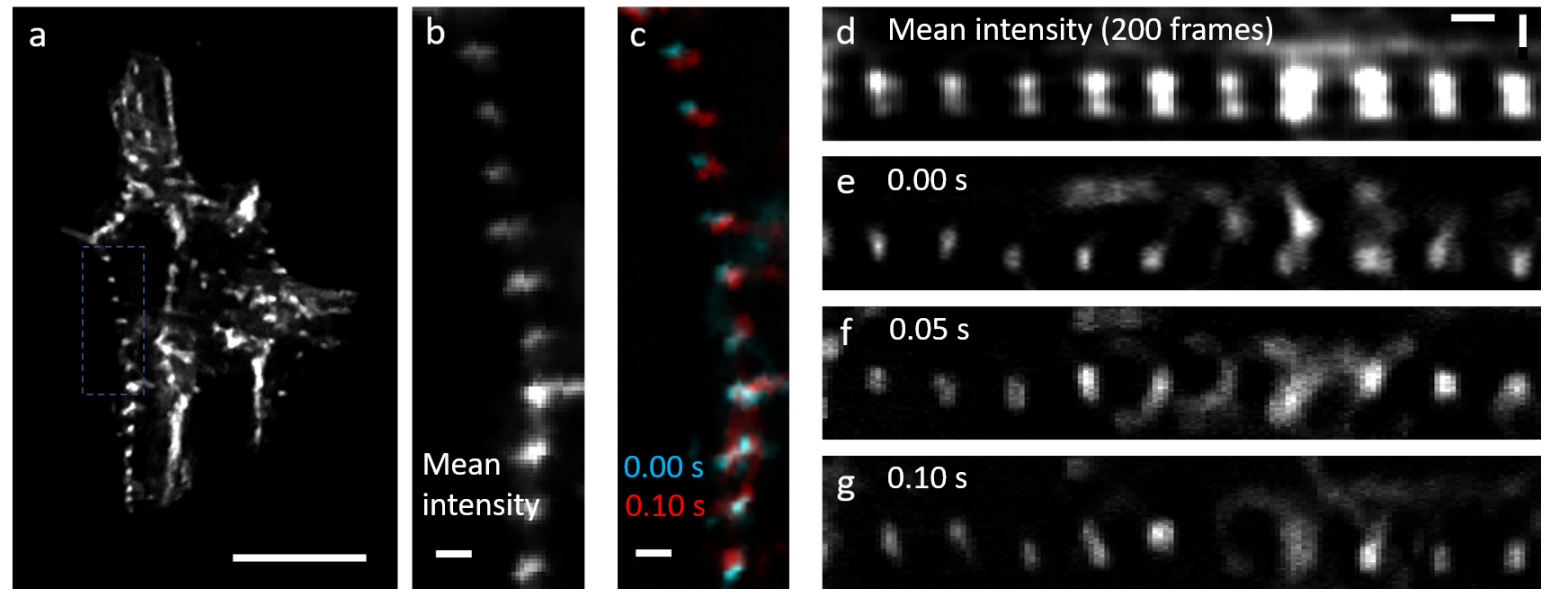

Figure 4.5: $\mathrm{A}$ is the z-axis mean projection image of the first frame. The blue dotted rectangle was cropped to enlarge the mean projection in the z-axis direction $(b, c)$ and the mean projection in the $\mathrm{x}$-axis direction $(\mathrm{d}, \mathrm{e}, \mathrm{f}, \mathrm{g})$. $\mathrm{B}$ and $\mathrm{d}$ are the averages of every frame, and $\mathrm{c}$ is the overlap of the two frames having the most positional difference (1st: cyan, 2nd: red). e, f, and g are the results of comparing the first, second, and third frames. Compared to the movement on the XY plane, it can be seen that the movement in the $\mathrm{z}$-axis direction is more complicated.(scale bar: $10 \mu \mathrm{m}$ in a, $1 \mu \mathrm{m}$ in b and c, $1 \mu \mathrm{m}$ lateral, $500 \mathrm{~nm}$ vertical in $\mathrm{d}, \mathrm{e}, \mathrm{f}$ and $\mathrm{g}$ 

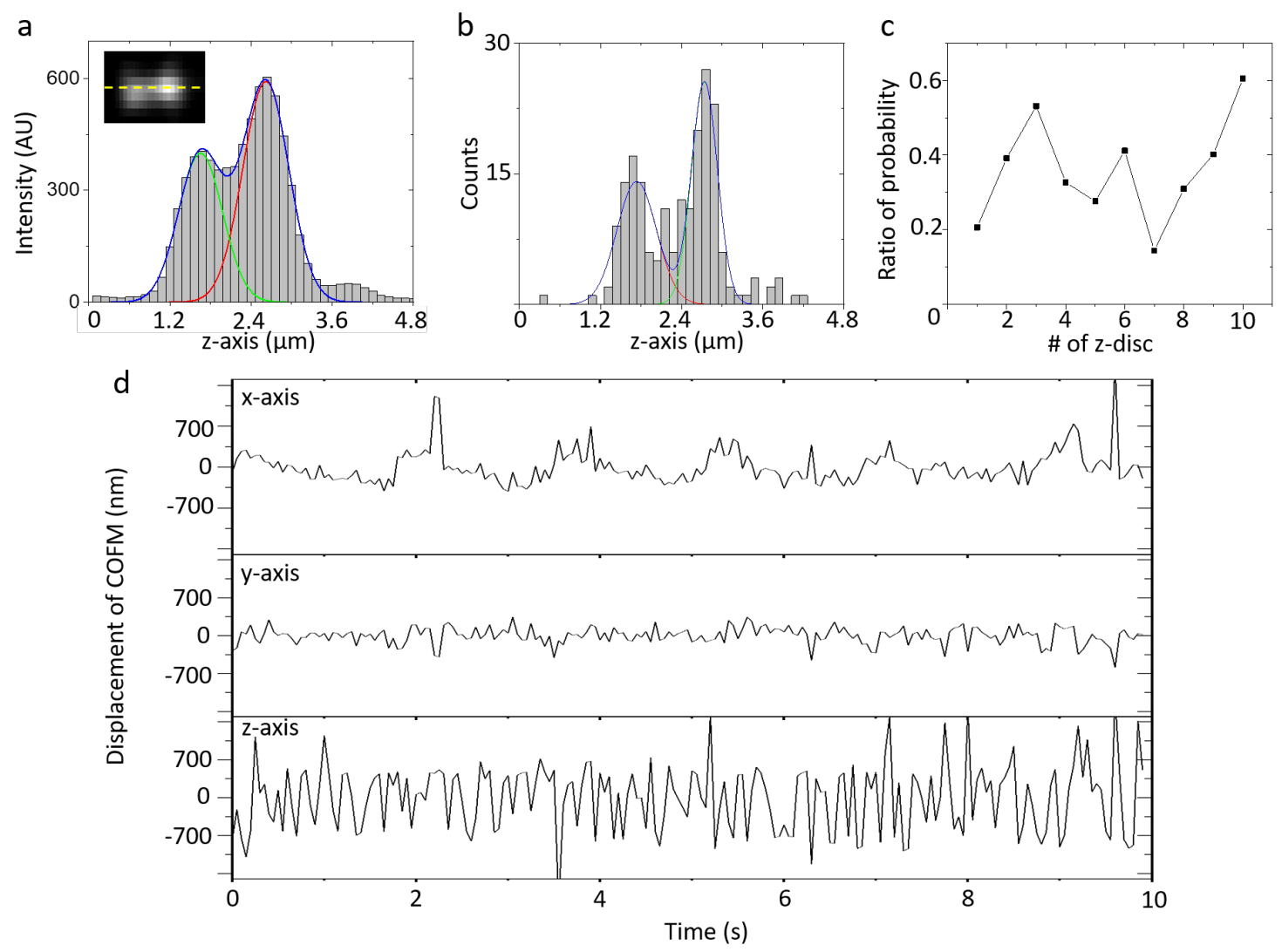

Figure 4.6: $\mathrm{a}$ is the cross section of a single Z-band in a subset. By fitting the cross section with two Gaussian functions, the z-axis coordinates of the most probable location of ACTN-2 were calculated. (peak center: $2.62 \pm 0.01 \mu \mathrm{m}, 1.65 \pm 0.02 \mu \mathrm{m}$ ). The second histogram is about the z-center of fluorescence mass for each frame (peak center: $2.74 \pm 0.02$ $\mu \mathrm{m}, 1.74 \pm 0.04 \mu \mathrm{m})$. The ratio between two probabilities of observing in each position for $10 \mathrm{Z}$-discs were plotted. The COFM movement of single Z-disc along each axis was plotted over time. The displacement on $\mathrm{x}$-axis has strong periodicity, but movement on $\mathrm{y}$-axis is smaller than on $\mathrm{x}$-axis. Th motion on z-axis is stronger and faster than other motions.

For the analysis of both distributions, cross-section intensity and COFM were analyzed. First, the cross-section intensity along the z-axis of a single z-disc is shown in figure 4.6. It is shown in graph a of figure 4.6. Two peaks were found in the cross-section intensity, and Gaussian functions were used to find the maximum points of the two peaks. The vertical position of the measured intensity maxima was compared with the vertical position of the COFM. COFM was also calculated for all frames of the same z-disc images. The vertical positions of the COFM were plotted as a histogram, and the distribution was compared with the mean fluorescence distribution. The most probable positions of 
COFM were equivalent to two positions of intensity maxima. Based on this result, we can infer that ACTN-2 mainly exists in two vertically divided spaces.

Additionally, the positional change on each axis of the COFM over time was also analyzed. The change of COFM along the $\mathrm{x}$-axis showed the periodicity by the two-dimensional beating motion. A relatively small movement was observed also along the y-axis. The displacement of the z-axis rapidly changed to a distance of about $1 \mu \mathrm{m}$ regardless of the beating period of $1.75 \mathrm{~Hz}$. However, the movement along the z-axis was too fast to define the exact frequency. From this result, we can also infer that the distribution of ACTN-2 constituting a single z-disc has a fast vertical motion.

\subsection{Discussion}

These dynamic changes in ACTN-2 position tend to be inconsistent with the role of Zdiscs, which are holding actin filaments.[78, 100] Z-discs have been considered a passive component because it acts as a fixed pillar in horizontal cardiomyocyte movement.[40, $41,88]$ However, in this research, the fast movement was observed. Because the rapid movement of constituent molecules can weaken the structure that holds actin filaments, this fast vertical motion can be considered as an opposite tendency to the major role of Z-discs.

Previous studies on the regulation of the constituent proteins of Z-discs have shown that protein recovery and regulation proceed very rapidly. [95, 96] Z-disc is a complex structure composed of several types of proteins, such as muscle LIM protein, desmin, and cypher. Recently, the molecular level structure of Z-disc is being studied. The study is the result of measurements using cryo-EM, and the result does not provide information on the dynamics and interactions between molecules of Z-disc. In this regard, the regulation mechanism of ACTN-2 has been recently reported, and the rapid regulation of proteins constituting Z-discs has also been reported. [82, 90, 96] It was also confirmed in the paper using FRAP that the fluorescence signal of the proteins constituting Z-discs recovers rapidly. To explain this fast fluorescence recovery time, it may be advantageous to maintain dynamic structure using fast regulation.

Therefore, the movement of ACTN-2 faster than the beating rate of cardiomyocytes can be a basis for explaining the regulation process of proteins constituting Z-discs. Further research is needed to explain the cause of this rapid vertical motion. Since ACTN-2 is 
distributed within Z-discs, the movement may be induced by the deformation of the $\mathrm{Z}$-disc, or this phenomenon may be a result of ACTN-2 regulation. The only conclusion of this study is that ACTN-2 is located in two vertically separated spaces and moves rapidly. Therefore, we need to solve the questions how the tension of the actin filaments is continuously maintained, and why the space is not continuous. To answer these additional questions, additional measurements must be made in different ways. Since only the movement of ACTN-2 was confirmed in this study, it may also be meaningful to conduct a study on the colocalization of ACTN-2 with other proteins constituting Z-discs. In addition, in order to simply find an answer to the spatial distribution of ACTN-2, it is also necessary to study the correlation between the distribution of various organelles and the distribution of ACTN-2.

In this study, the movement of cardiomyocytes was studied through 3D-SUPPOSe multi-plane imaging. Cardiomyocyte beating is generally considered as a planar motion. The planar motion is driven by active components of the cells. Many studies have been conducted on the movements of the active components, actin and myosin.[37] The interaction between actin and myosin induces the beating of cardiac muscle cells, and the movement of calcium ions plays an essential role.[30] However, studies on the role of other components such as ACTN-2 are relatively deficient.

A known role of ACTN-2 is to hold actin filaments and to maintain the tension of the A-band. [78, 100] However, ACTN-2 is a protein constituting Z-discs, and the protein is considered only as a target that marks the boundary of sarcomere that appears repeatedly in cells. Studies on the mechanism and reaction between other constituents are insufficient. Through this study, we were able to know the unknown distribution and movement of ACTN-2, and the dynamic characteristics of ACTN-2. The dynamic information are expected to be used importantly in the process of understanding ACTN-2 and Z-disc.

\subsection{Summary}

- Using the deconvolution multi-plane setup, I imaged the three-dimensional motion of a single cardiomyocyte.

- In a single cardiomyocyte's beating motion, I could find a clue of the vertical motion 
of ACTN-2.

- Using COFM calculation, I traced the motion of a single Z-disc.

- ACTN-2 molecules moved faster than the beating motion of a single cardiomyocyte in a single Z-disc. 


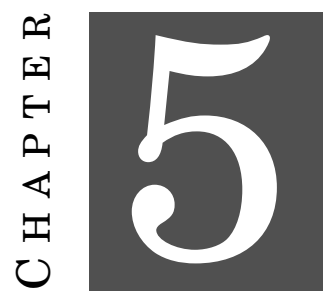

\section{Live Cell Imaging - Dictyostelium Discoideum}

\footnotetext{
$\mathrm{D}$
} ictyostelium Discoideum (Dicty) is a type of soil-dwelling amoeba, and it is commonly referred to as slime mold. The amoeba is considered as a model system for not only describing chemotaxis but also cell adhesion, pattern formation, evolution/multicellularity, or phagocytosis. The cells recognize the chemoattractant's gradient and are able to move towards the source.[45,62] Chemoattractants are inorganic or organic substances inducing chemotaxis effect in motile cells, and in the case of Dicty, the attractant is cAMP. These chemotactic ligands create chemical concentration gradients that organisms, prokaryotic and eukaryotic, move toward or away from, respectively.[118] Dicty moves in the chemoattractant direction using pseudo-pods, which are transient arm-shaped projections of eukaryotic membranes that develop in the direction of movement. In pseudo-pods, the actin polymer pushes the membrane as it grows, forming new pseudo-pods or splitting the ends of existing pseudo-pods. The pseudo-pod then pulls the body of the cell forward through the contraction of the actin-myosin complex in the cell cortex. Thus, the distribution and dynamics of actin and myosin molecules in cells provide information on chemotactical mechanisms. In order to find out this information, the distribution of actin in cells is imaged using actin binding protein to which a fluorophore is attached. 
The effect of Myosine and Actin networks on Dicty's chemotaxis is also studied using Myosine null cells designed to prevent the formation of regular actin networks.[36, 39] Myosin plays a key role in the process of actin filament building network and tension formation. [6] Therefore, actin does not form a proper network in myosin null cells, thus the usual cortical actin flow which induces the chemotactic movement of the cells is disturbed. [121]

In this chapter, I use 3D-SUPPOSe multi-plane microscopy to analyze actin flow inside myosin null cells. The direction of movement is in the two-dimensional plane, but the shape of the cell and the pseudo-pod formation are three-dimensional motion. Therefore, the 3D-SUPPOSe multi-plane setup is a suitable system to study the flow and interaction of molecules involved in pseudo-pod formation.

However, the extent of the cells along the z-axis is sometimes above 10 micrometer and therefore tends to exceed the multi-plane setup range. Therefore, I have prepared a sample preparation method to overcome this problem. By flattening the dicty cells, I fitted the height of the cells into the field of view of the microscope. Thus, this flattening approach allows us to image an entire cell at the same time. Accordingly, the threedimensional distribution of actin within live dicty cells were measured and analyzed. Furthermore, flattening down to 2-3 micrometer was proven to be noninvasive to dicty cells in the past.[115] Using the same principle as the method described in the paper, I prepared a more simplified cell flattening device to study cells. The purpose of this study is to explain the relationship between actin flow and chmotactical motion by imaging the motion of a dicty using a 3D-SUPPOSe multi-plane setup and the flattening device.

\subsection{Method}

\subsubsection{Cell Preparation}

Dicty cells were provided by the Bodenschatz department of the Max Planck Institute for Dynamics and Self-Organization. GFP has been fused to the actin-binding protein LimE by genetical mutation. LimE binds freshly polymerised filamentous actin (F-actin), and the distribution of $\mathrm{F}$-actin can be measured using a multi-plane microscope. The labeling scheme and specific binding affinity of LimE to F-actin were explained in the papers.[19, 34] The Measurements were started approximately six hours after starvation initiation to satisfy the active chemotaxis condition. 


\subsubsection{Microscope}

The field of view is illuminated using a LED illumination system (Lumencore, Spectra $\mathrm{X})$. The illumination corresponds to an epi-luminescence wide-field microscope. For excitation of GFP, a cyan LED (emission between $490 \mathrm{~nm}$ and $500 \mathrm{~nm}$ ) was used, and fluorescence was recorded using a $500 \mathrm{~nm}$ long-pass emission filter. Images were recorded by temporally synchronized two CMOS cameras (Hamamatsu, and recording speed was from 0.4 to $0.8 \mathrm{~s}$ per frame. An epi-fluorescence illumination is obtained by focusing the light at the back focal plane of a 60x water immersion objective (UPLSAPO60XW, 1.2 NA, Olympus). The fluorescence emission is collected by the objective in the backreflection and then is transmitted through a Quad-band beam splitter (Quad Line Beamsplitter R405/488/561/635 lambda/5, Semrock). A multi-plane prism is incorporated in the detection path, which enables to detect eight distinct but equally-spaced image planes along the optical axis (the depth of sample) [32]. These image planes exit the prism as two sets of four adjacent images. Two sCMOS cameras (ORCA-Flash 4.0 V2, Hamamatsu) record the image planes synchronously. A further lateral magnification factor of 1.33 is achieved using a telescope system comprised of two lenses with focal lengths of $150 \mathrm{~mm}$ and $200 \mathrm{~mm}$.

\subsubsection{Cell Flattening Device}

The height of the field of view is limited. When the numerical aperture and lateral magnification of the objective are 1.33 and $60 \mathrm{x}$, the distance of each image plane is 360 $\mathrm{nm}$. Therefore, the height of the image space is about $3.2 \mu \mathrm{m}$. However, the height of normal dicty cells is more than $10 \mu \mathrm{m}$. Due to the limitation of image volume, the whole cell cannot be measured at the same time. The height of the field of view can be changed when we use the objective lens of low magnification, but it decreases the spatial sampling rate.

Because of this reason mentioned above, the cell flattening device was applied. The double-layered flattening device has been developed by Unger et al.[110] Initially, the flattening device was a single-layer device. Later, the device has been modified to a double-layer system. Cells move through the lower channel, and we can control the height of the lower channel by controlling the volume of the actuation channel (upper channel, see figure $5.1 \mathrm{a}$ and c).[115] 

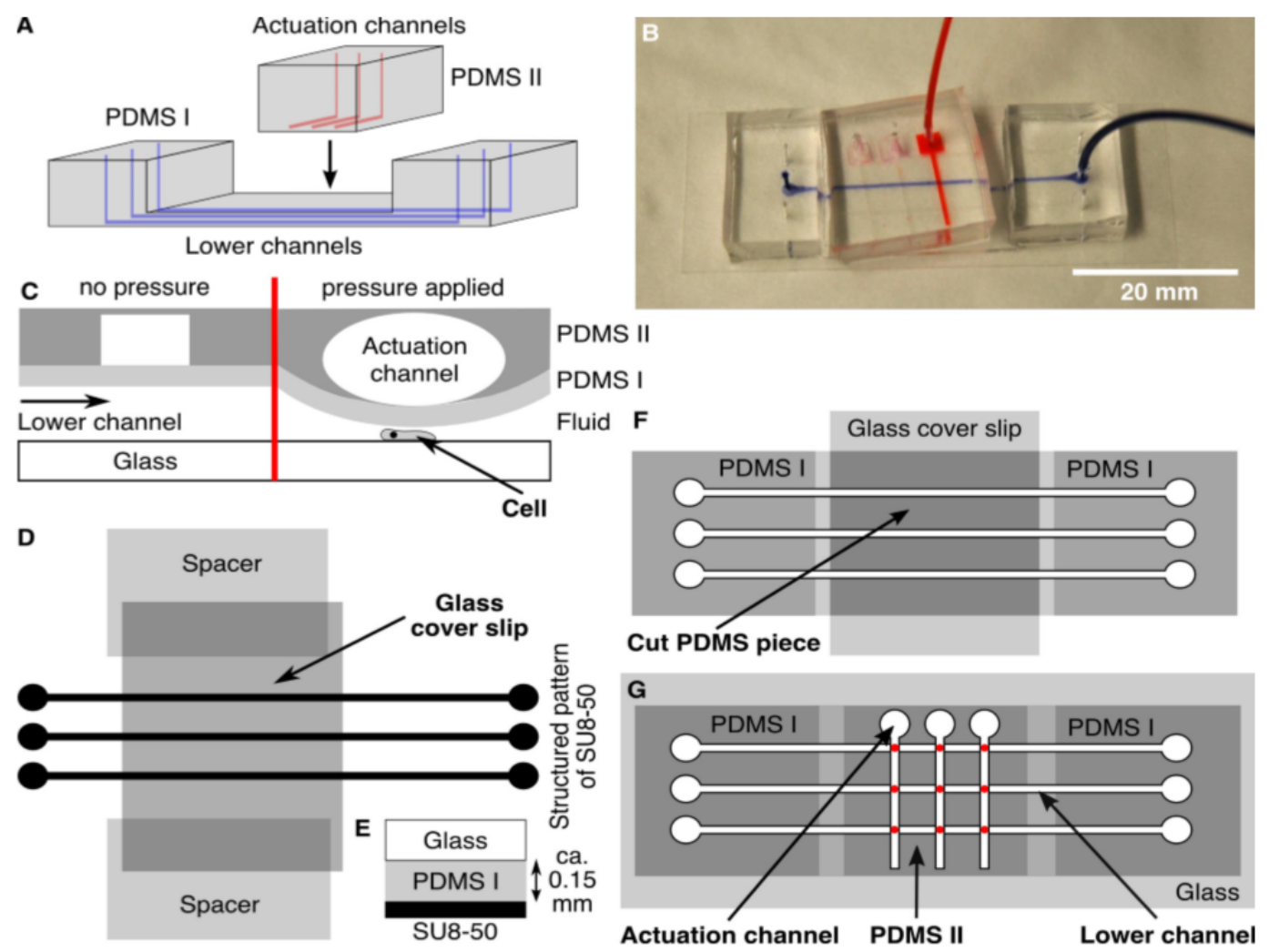

Figure 5.1: Design of the double layered cell flattening device (A) The closed-end actuator consists of a lower layer (PDMS I) with channels containing the cells (blue) and an upper layer (PDMS II) with the actuation channels (red). (B) Photograph of the assembled closed-end actuator filled with ink (actuation channel in the red and lower channel in blue). (C) The principle of operation for the closed-end actuator. (D) To create the lower layer, PDMS was poured onto the wafer. Then a cover slip was placed across the pattern. This cover slip rests on two other cover slips, which act as spacers that define the height of the PDMS layer (E). This layer will separate the lower and the upper channels. (F) After curing and cutting the PDMS, the middle section was cut out and stored. The first cover slip was replaced by a second cover slip and the cut PDMS piece is placed in its former position to provide stability during plasma cleaning. (G) Illustration of the assembled double layer device. The lower layer (PDMS I) contains the microfluidic channel filled with the cells. The upper layer (PDMS II) contains the closed-end channel which applies pressure to the channel below. The red spots in $(G)$ mark the overlapping areas, in which flattening of the cells occur. [115]

The design is based on PDMS-based microfluidic channels. To prepare this device, a clean room and well-designed master for micro-patterning are required. This facility and equipment are expensive, and the process for beginners to learn this skill is also difficult.

For the experiments on the multi-plane microscope, I developed a simpler version of 
the device. The fluidic channel was prepared with PDMS (Sylgard 184, Dow Corning), disposable Petri dish, and scotch tape. For the lower channel, two sheets of tape were used. The height of the tape is around $70 \mu \mathrm{m}$, so the height of the lower channel is less than $150 \mu \mathrm{m}$.

A

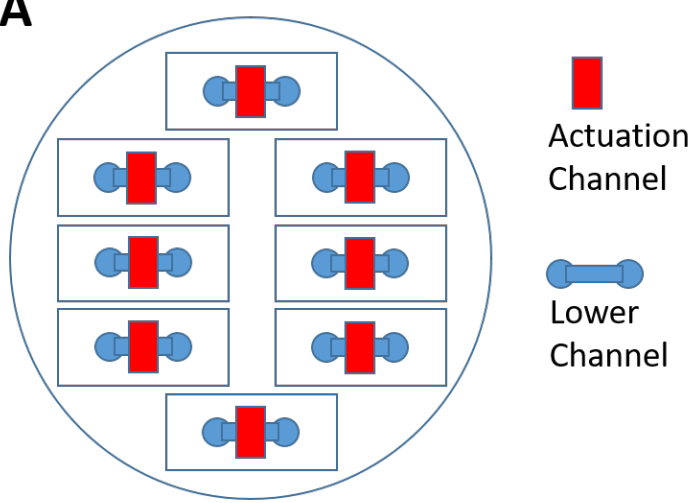

Design of Scotch tape device
B

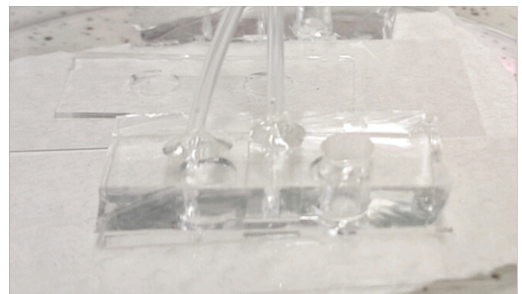

C

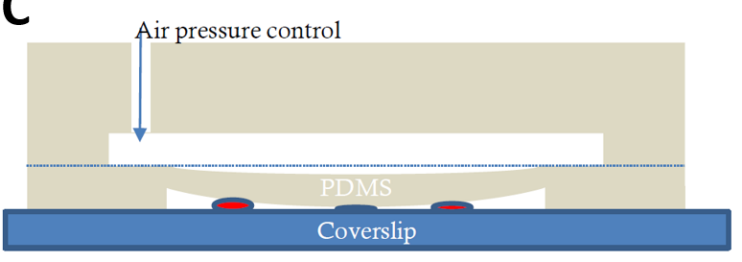

Figure 5.2: Modified design of cell flattening device. (A) Illustration of the master. The circle is the size of petridish, the red rectangle is the size of the upper channel, blue rectangle and circle are a lower channel, and small empty square is the unit size of device. (B) Picture of the device. (C) Illustration of section
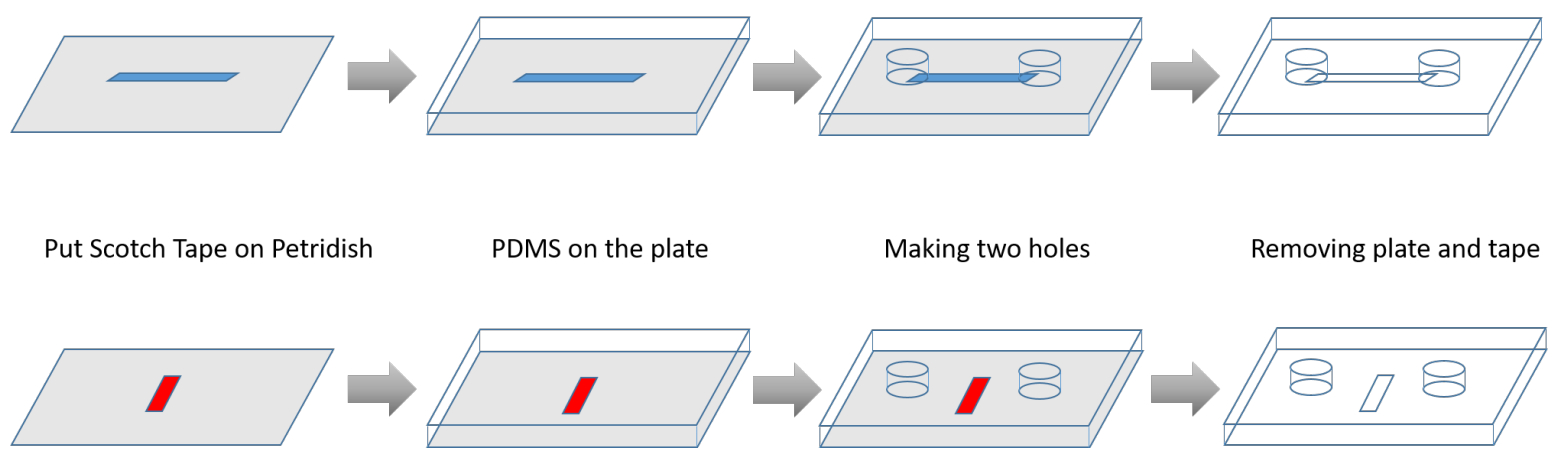

Figure 5.3: A brief description of the making process. PDMS blocks for each layer were made separately and combined.

The master of the lower channel was prepared with the two stacked scotch tape sheets. For the upper channel, ten sheets of tape were used. On the master of the channels, 
PDMS solution was poured and kept in a vacuum chamber until efficient degasing was observed. The mold was cured at $70^{\circ} \mathrm{C}$ in an oven.

\subsection{Results}

\subsubsection{Height Profile - Cell Flattening Effect}

To evaluate the performance of the cell-flattening device, the height of the dicty cells was measured. The height of the cells was imaged using a multi-plane setup. To evaluate the performance of the cell-flattening device, the sample stage was scanned along the z-axis with a step size of $200 \mathrm{~nm}$. By comparing the height of the dicty before and after the use of the flattening device, it was confirmed that the height of the channel is controlled enough to fit the FOV of the multi-plane setup. Through the vertical cross-sectional image, the change in the height of the dicty before and after pressing was compared. To compare the heights, I selected the areas corresponding to the top $20 \%$ brightness. Before the cell was compressed, the height was more than $10 \mu \mathrm{m}$. After pressing the cell, the height decreased to less than $4 \mu \mathrm{m}$.

The performance of the device was also confirmed by the two-dimensional shape change of the cell (see figure 5.4). After pressing the cells, the shapes were changed in the z-projection image. Before pressing, the shape was almost circular, but after pressing, the contact area was increased and the shape became more irregular. In order to compare the changes in the overall cell morphology, the image data were rendered as $3 \mathrm{D}$ volume images. (see figure 5.5) In the 3D result of a typical Dicty image, the cells were domeshaped, but the top structure was irregular, and the height was more than $10 \mu \mathrm{m}$. However, as the flattening started, the irregular structure at the top side decreased and the contact area increased. After flattening, the height was about $4 \mu \mathrm{m}$, and the top and bottom were completely pressed down. 


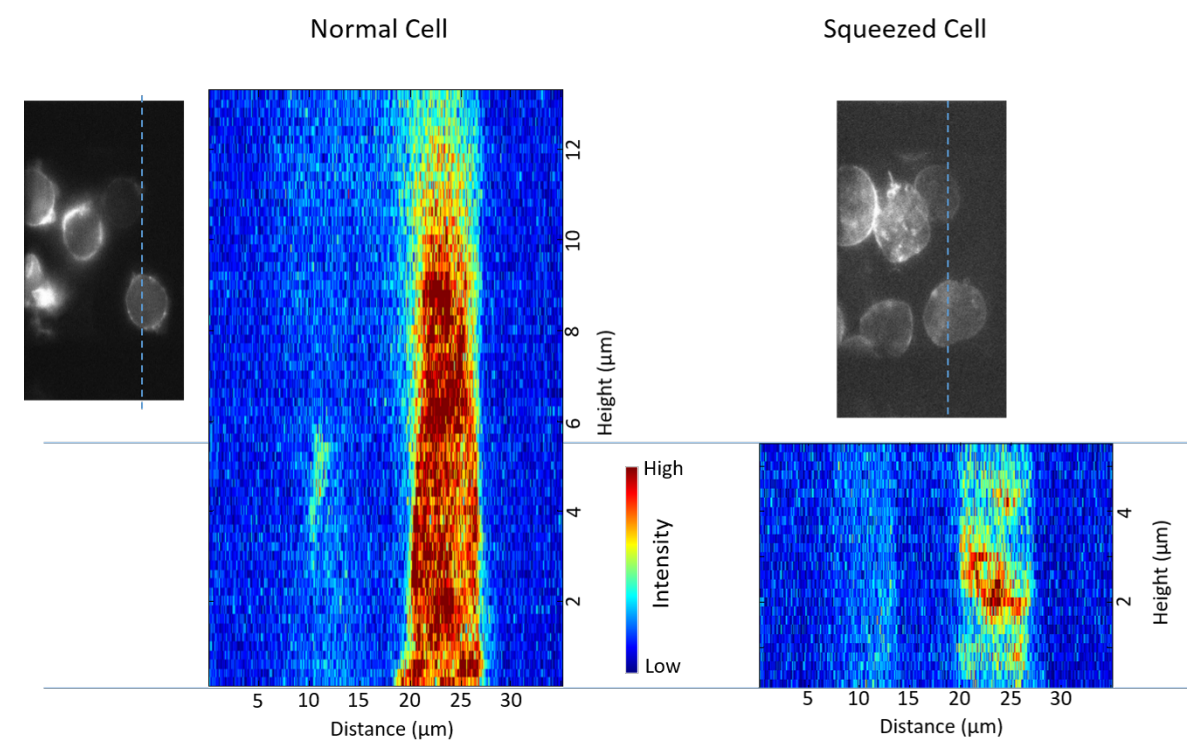

Figure 5.4: Change of the height and shape of cells. Left: z-projection image and section image of normal cells. Right: z-projection image and section image of squeezed cells. (pixel size: $80 \times 80 \times 200 \mathrm{~nm}$ ) To compare the two section images, the images were resized in the same ratio.
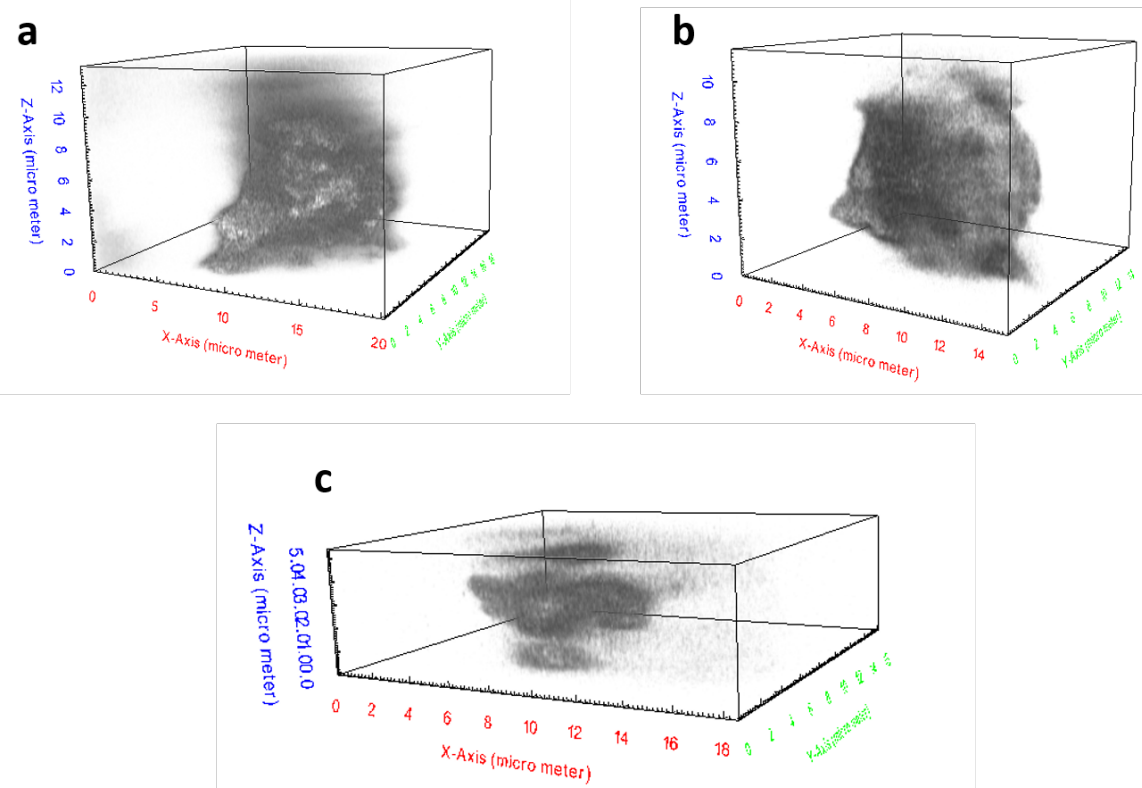

Figure 5.5: 3D rendering result of myosin null Dicty cells. a) is the result of the uncompressed cell. The height is over $10 \mu \mathrm{m}$. b) and c) are the result of the pressed cell. The height was reduced over $10 \mu \mathrm{m}$ to less than $5 \mu \mathrm{m}$. 


\subsubsection{Evaluation of Effect on Cell Vitality}

The change of cell height controlled by the cell-flattening device was clearly shown. However, the vitality of the pressed cells is an important issue to consider. To diagnose Dicty's activity, I observed chemotactical behavior of the cells. Chemotactical behavior is a complex process that detects chemoattractants and moves cells by actin flow inside, and this behavior is also an important phenomenon in the life cycle of dicty. Therefore, in order to diagnose the vitality of the cell, I observed the pattern of cell activity and movement.
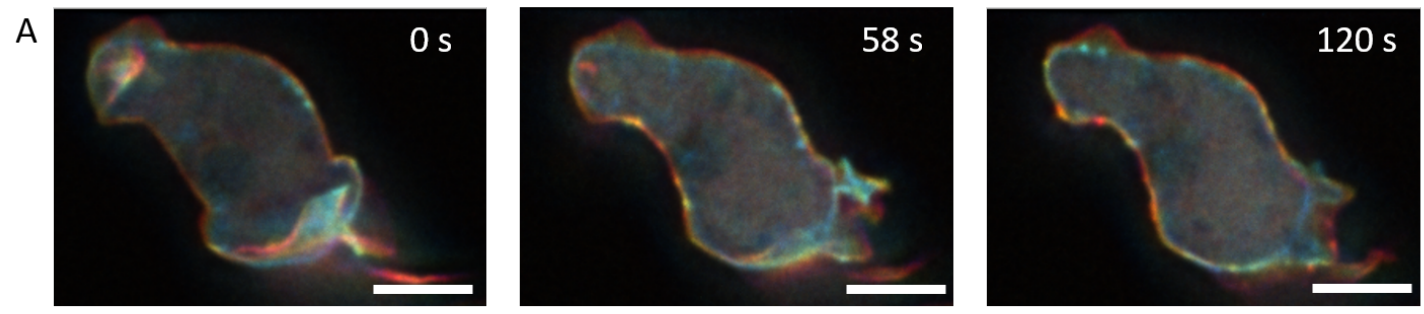

$\mathrm{B}$
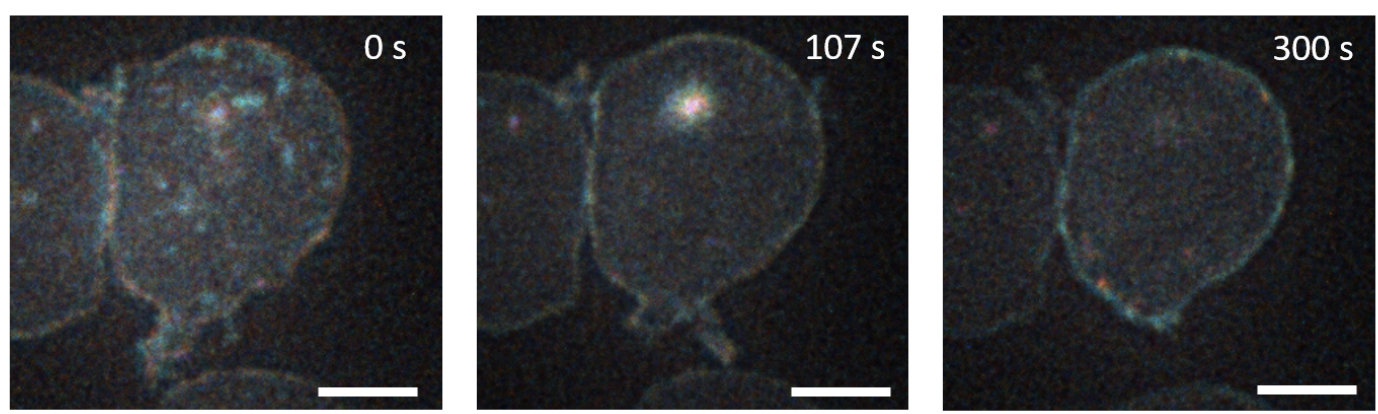

C
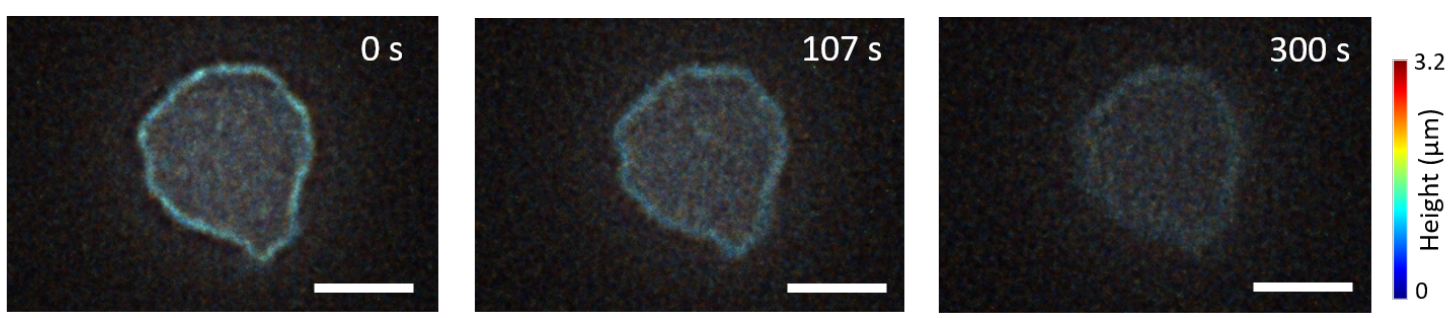

Figure 5.6: Activity of each cells is compared. A are control images of cell before compression. The movement of actin molecules is clearly observed inside and at the cortical periphery of cells. B is the images of cell slightly pressed: a lot of vertical F-actin flows and small movement of pseudo-pods are observed. $\mathrm{C}$ is an image series for an exemplary strongly pressed cell. No movement of F-actin was found in $\mathrm{C}$, and fluorescence signal was bleached out. The size of the scale bar is $5 \mu \mathrm{m}$. (The different color means the different height.)

Native dicty cell before flattening is very active (see figure 5.6 A). F-actin induces pseudopod extension, and some pods are folded into cells to increase cell area. After 58 seconds, 
the cell releases the pseudo-pod to move again, and it continues to move. The area occupied by the active pseudo-pods was about $10-20 \%$ of the cell boundary, and the fluorescence brightness was also strongly maintained during the recording time. Next, the pressed cells showed actively moving pseudo-pod outside the cell like before pressing (see figure $5.6 \mathrm{~B}$ ). However, the area occupied by the pseudo-pod was reduced to less than $10 \%$. This seems like the effect of height reduction, and pseudo-pods of small size seen in the plane continuously repeat the formation and deformation. Although the total fluorescence intensity decreased slightly, the intensity was still sufficiently high for a measurement time of about 300 seconds. However, when the cells were pressed perfectly without any space, the cells could no longer move (see figure 5.6 C). The brightness decreased during the measurement time due to photobleaching. Through the fluorescence intensity change and the movement of cells, we can evaluate the fatality of pressing on cells without sufficient space to move. However, it has been also confirmed that when sufficient space is secured, the pressure is not fatal on the cell vitality.

\subsubsection{Temporal Relation between Actin Movement and Geometric Center of a Cell}

To measure the change of actin molecule distribution, 23 cells were imaged using multiplane setup. The frame rate of the video presented in this thesis is $0.8 \mathrm{sec} /$ frame. After imaging, six interpolation processes were performed along the z-axis to achieve a uniform aspect ratio in all directions. The images were then deconvolved using 3D-SUPPOSe. The image expressed the dynamic 3D motion of the pseudo-pod. Then, the cells were pressed to a non-invasive height in the field of view by the previously verified sample preparation method. Images were recorded while ensuring adequate vitality during the measurement time.

Myosin null cells cannot express myosin II. In the absence of Myosin II, F-actin does not form a regular actin network. Instead of the regular actin network, actin molecules in the cortex play an important role in chemotactical motion. Due to the difference on the role of actin molecules, Myosine null cells move more slowly than normal dicty cells,[121] and these cells are good candidates for elucidating the relationship between myosin, cortical actin and chemotactical motion.

Centroid and COFM were analyzed to study actin flow in Myosin null Dicty cells. In the previous papers about Dicty, many explanations were made based on the geometric 
center to confirm the positional change of cell migration.[10, 12, 97] The geometric center assumes uniform fluorescence intensity as described in 2.5.2. However, in the case of myosin null cells, F-actin forms a non-uniform flow within the cell, and this flow is expressed by the brightness of fluorescence. Therefore, the difference between the centroid and COFM explains the movement of the fluorophores inside of cells. This was used to measure the F-actin movement change over time.

a

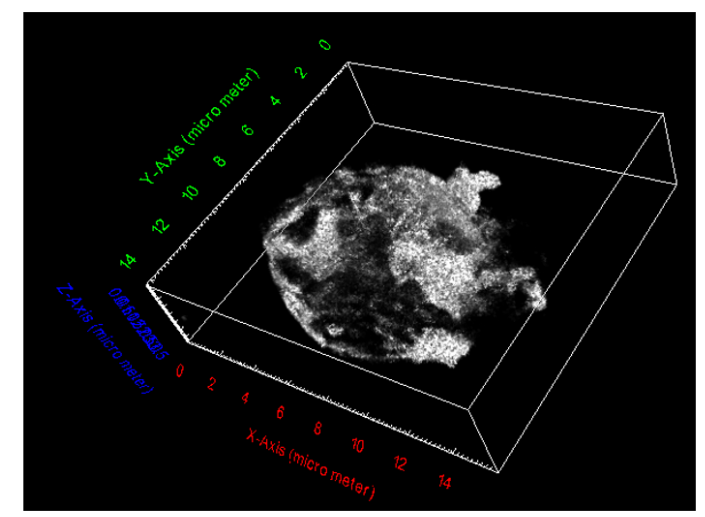

b

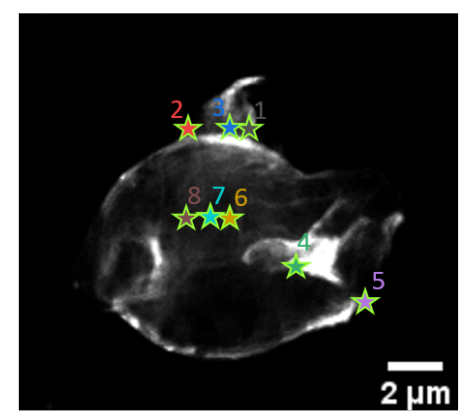

C

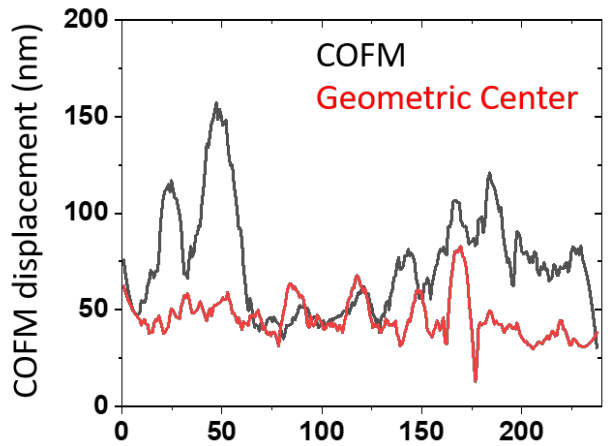

d

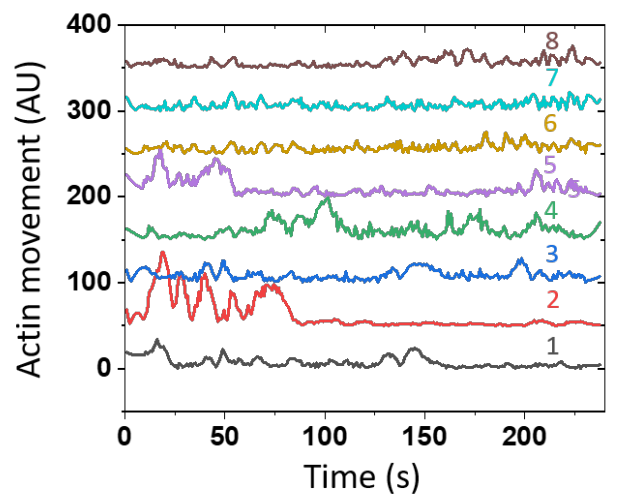

Figure 5.7: (a) 3D rendered result of the dicty image. The image clearly shows the shape of protruded pseudo-pods. We can find some empty space probably where organelles are placed. (b) The regions of interests (ROIs).(11x11x11 voxels for each ROI) I selected some areas and analyzed the intensity change and flow of actin at the regions. (c) Comparison between the amplitude of COFM and centroid of the cell. The position of centroid is moving slowly and continuously relatively than COFM. The graphs were smoothed by Savitzky-Golay filter. (window size: 15 points) (d) The amplitude of estimated flow in the selected regions. The same color coded as in (b). We can find some spikes on the graph of the signals of the areas near pseudo-pod.

The difference between the two parameters is clearly shown in the graphs of COFM and centroid. Centroid maintains a value in a certain range. Conversely, COFM shows two sharp increases before $40 \mathrm{~s}$ and $170 \mathrm{~s}$ (see figure 5.8). c) The increase in COFM 
without change in centroid means that a significant density change occurs inside the cell. Compared with the motion of the image, the first increase was related to the formation and deformation of pseudopods. In the second increase, there was no additional pseudopod change, so it seems that the change in the concentration of F-actin inside the cell caused the COFM difference.

To find the cause of the difference between COFM and Centroid, the optical flow estimation method described in 2.5.2 was used. Eight characteristic regions were selected to compare the flow patterns, and the absolute values of the estimated velocities at the selected locations were compared. Eight characteristic regions are classified into the regions with three different motions, each region having three different motions: the active region near the active pseudo-pod, center of the cell, and the active region near the membrane. The velocity change over time was strongest in the protruding pseudo-pod region.
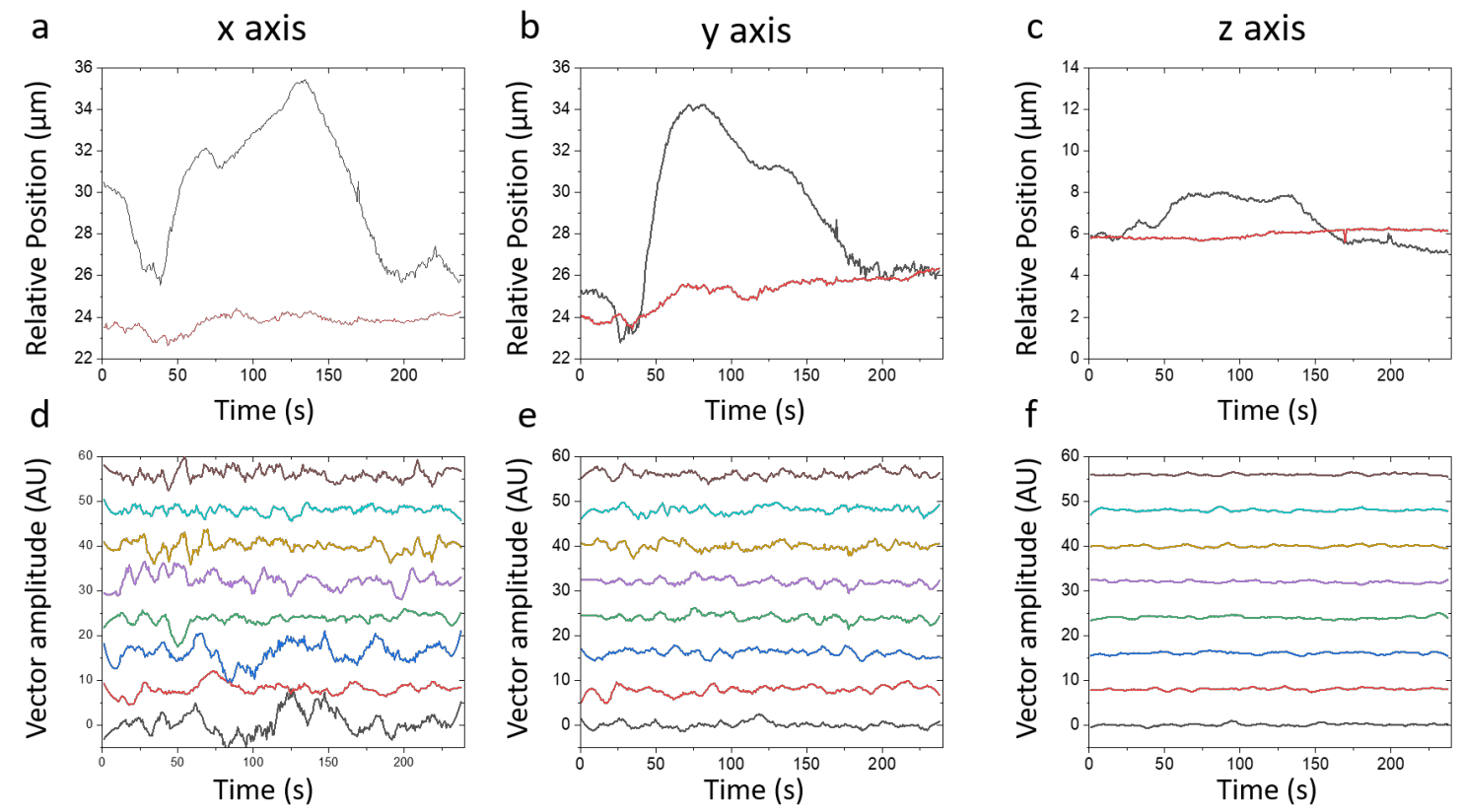

Figure 5.8: The $\mathrm{x}, \mathrm{y}, \mathrm{z}$ components of the flows in the selected regions were plotted. (a), (b), (c) are the $\mathrm{x}, \mathrm{y}, \mathrm{z}$ components of positions of centers. The black lines are the position of COFM, and the red lines the position of centroid. In (d), (e), (f), the same colors with the figure 5.8 were coded for the same regions. The movement on the z-axis is relatively smaller than other components. This phenomenon is related to the confinement of vertical volume. Before $100 \mathrm{~s}$, The cell shows dynamic movement, and the flow is relatively bigger than after $100 \mathrm{~s}$. After $100 \mathrm{~s}$, we can also find some additional flow in the areas near the pseudo-pods. (black and blue lines on the bottom) 
Next, the flow of F-actin was analyzed by dividing it into components for each axis of the three-dimensional space. First, centroid and COFM movements were analyzed by dividing them into $\mathrm{x}, \mathrm{y}$, and $\mathrm{z}$-axis components. The tendency of the central position to move steadily was the same for all axes. The center of the y-axis moved the most, and the movement of the z-axis was very small. The small change in the $\mathrm{z}$-axis is due to the vertical volume limitation using the cell flattening device. However, in COFM, the position changed a lot, and the direction was not constant. The velocity varied in both the $\mathrm{x}$-axis and $\mathrm{y}$-axis, and the amplitude was greater than the center. The $\mathrm{x}$ and $y$ components of the COFM changed in the range of about $10 \mu \mathrm{m}$. The COFM along the $\mathrm{z}$-axis also varied in the range of about $2 \mu \mathrm{m}$. Through the difference between centroid and COFM, it is possible to infer the time when the movement of F-actin is active. However, this phenomenon is related not only to the movement of pseudopods, but also to the movement of organelles inside the cell. This is because the COFM may change due to the movement of organelles in regions where actin binding protein cannot access. In order to more directly understand the molecular flow trends in each region, the optical flow estimation results were analyzed by dividing the $\mathrm{x}, \mathrm{y}$, and z-axis components. Here, the movement of the $x$-axis component was the largest. The reason is that the cell has a weakly polarized shape in the x-axis direction, and a pseudo-pod outside the cell membrane is selected parallel to the $\mathrm{x}$-axis. The movement along the $\mathrm{y}$-axis was smaller than the movement along the $\mathrm{x}$-axis. Motion along the $\mathrm{z}$-axis still had little motion due to spatial constraints. However, weak changes were observed due to the movement of the pseudo-pod or the movement of organelles. Through this, it was confirmed that there is a correlation between the movement direction of pseudo-pod and Dicty and the movement of F-actin.

To better understand the trend of the measured velocity at each selected location, the measured video was divided into three parts over time. The division criterion is the point at which the morphological change of the cell appears. First, small pseudopods appeared or disappeared most prominently at intervals between about 0 and 80 seconds. Second, large pseudo-pod formation along the y-axis occurred between approximately 80 and 180 s. Finally, after about 180 seconds, the cell morphology was relatively stable compared to before 180 seconds.

The calculated velocity amplitude of $\mathrm{F}$-actin was compared for the three different time ranges. As a result of analyzing the actin flow for each event, the actin movement 
appeared the fastest in the first period from 0 to 80 seconds. In addition to the flow near the pseudo-pods, the intracellular actin movement was also stronger than in other periods. In the second period, overall movement decreased, but movement was still active. The last period showed a stable trend except for the cell interior and part of the cell membrane. This trend is more evident in the difference between COFM and centroid. Interestingly, when pseudo-pods were formed in other regions (second period), F-actin flow in the membrane was strong without additional pseudo-pod formation.
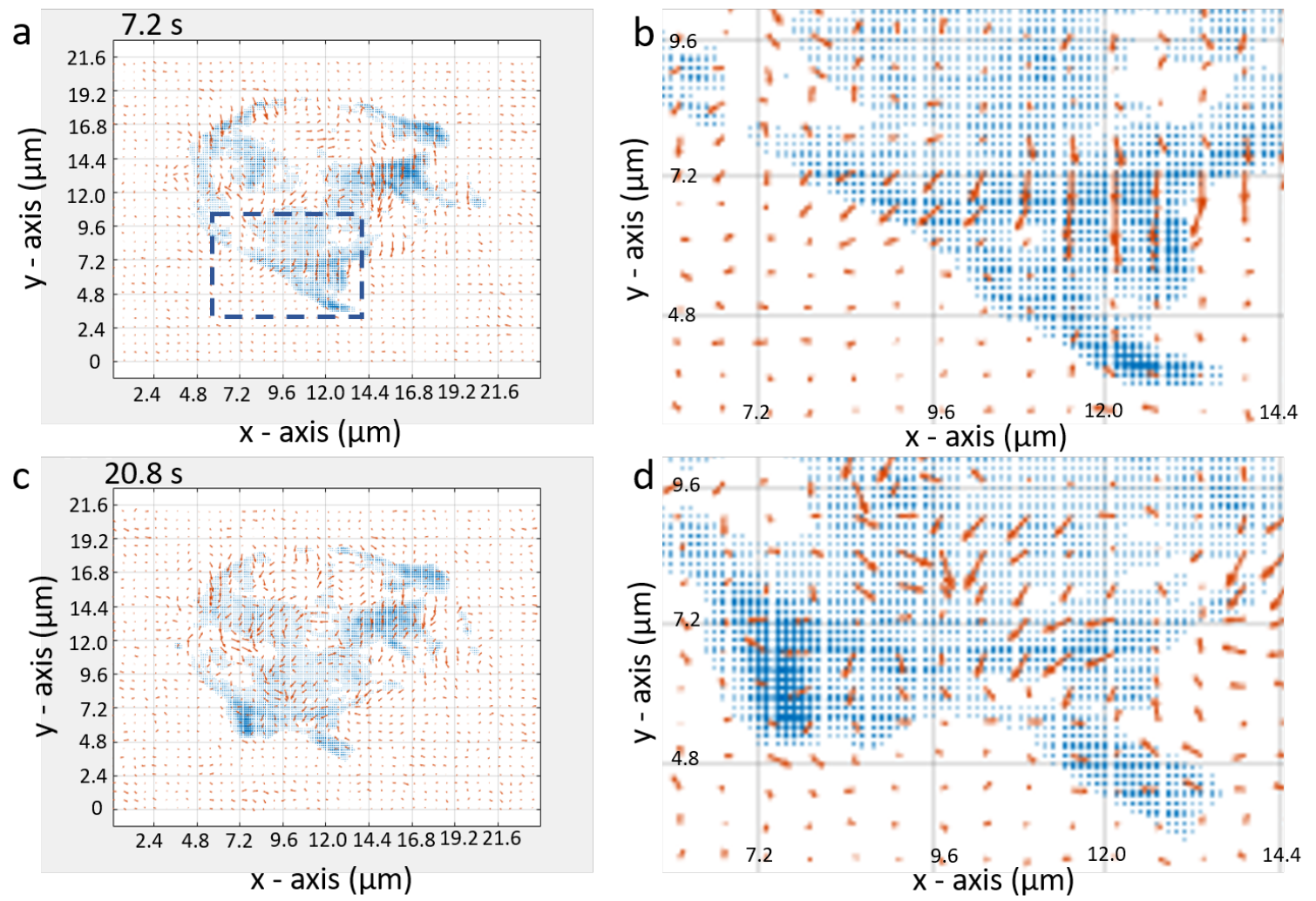

Figure 5.9: The actin flow in the whole area was plotted. The red arrows mean the direction and amplitude of the actin flow. The blue dots show the fluorescence intensity. The images show the movement within the $\mathrm{x}, \mathrm{y}$-plane because the vertical movement of actin was relatively smaller than lateral motion. In $7.2 s$ (a and b), we can find some strong motions inside the cell. In the area of organelles and pseudo-pods, the flow is stronger than other areas. I blowed up the small area of pseudo-pod. (b) shows more clearly the flow near the pseudo-pod when the motion is active. At $20.6 s$ (c and d), the pseudo-pod was deformed, and a new pod was formed. In the process, actins moved out from the old one and moved into new pod. However, the flow was not constant. The actin made some fluctuation in the flow.

The estimated actin flow in figures 5.9 and 5.10 was plotted to visualize real-time changes 
over the whole cell region. Since the z-axis movement is relatively small, the plot is projected onto the xy plane. As a first characteristic phenomenon, I observed that small pseudopods were formed and decomposed transiently, followed by expansion of other large-like pods. In this process, called tip splitting, the flow of F-actin was not fully temporally synchronized with the movement of the pseudo-pod. One enters the newly formed pseudo-pod and the other exits the modified pseudo-pod. Obviously, actin flow into the emerging pseudo-pods was observed but the direction of flow was not fully synchronized with the direction of the pseudo-pods. This suggests that the supply of cortical F-actin and the formation of pseudo-pods do not occur in the same time.
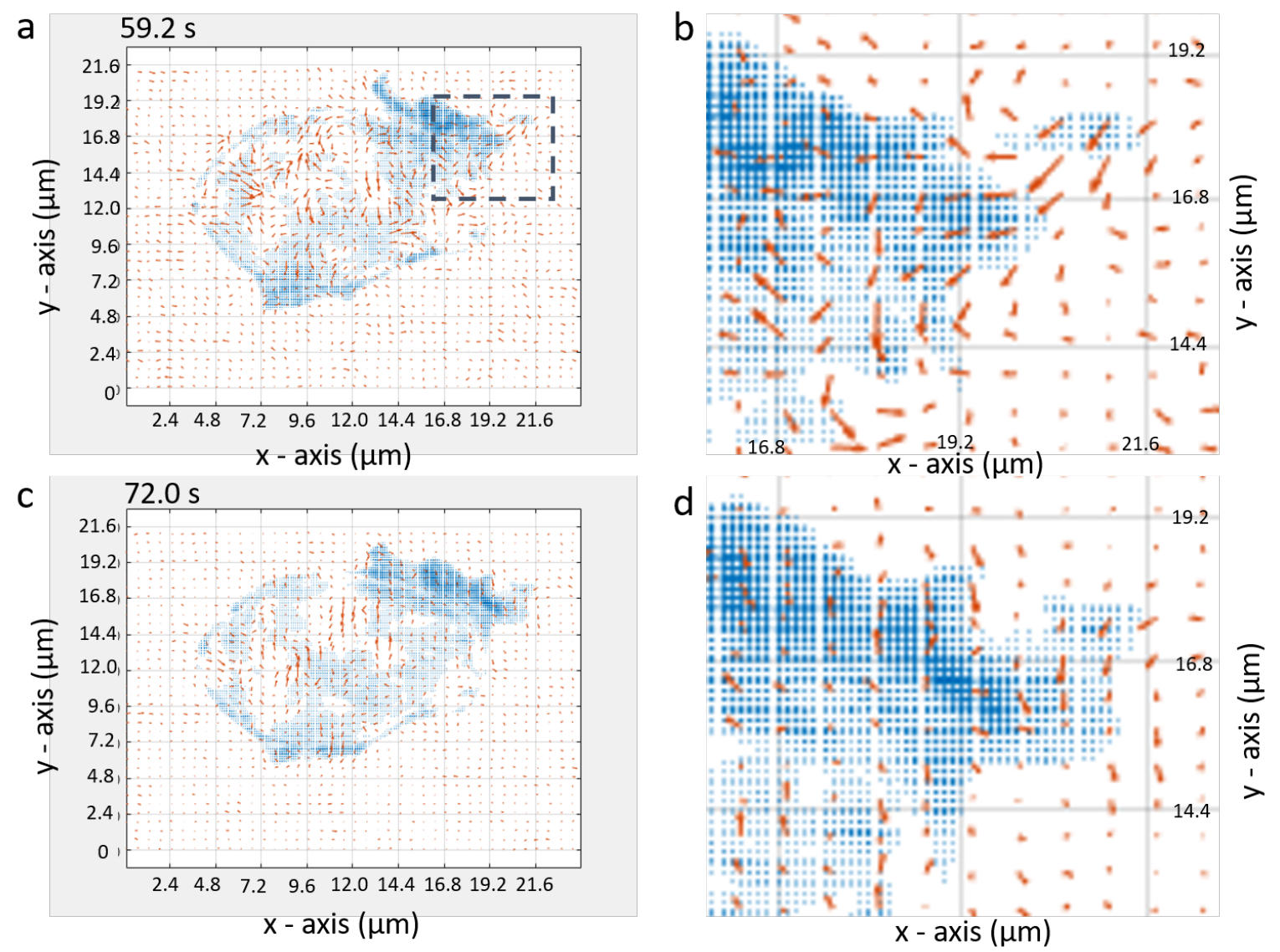

Figure 5.10: The image was plotted in the same manner as the figure 5.9. In this case, the flow near pseudo-pod was also strong in the time frame of $59.2 \mathrm{~s}$, (a) and (b). The pod was shrinking, and the flows are directed to the inside of the cell. In the next frame of $72.0 \mathrm{~s}$, the amplitude of the flow was reduced, and the flows directed inside the cell. However, the new pseudo-pod was extruded outward, so this is an example of temporally mismatched flow with the pseudo-pod formation. 
The difference between the two parameters is clearly shown in the graphs of COFM and centroid. Centroid maintains a value in a certain range. Conversely, COFM shows two sharp increases before $40 \mathrm{~s}$ and $170 \mathrm{~s}$ (see figure 5.8). c) The increase in COFM without change in centroid means that a significant density change occurs inside the cell. Compared with the motion of the

\subsection{Discussion}

The new flow estimation method was used to track the F-actin flow inside the Dicty cell. In particular, the occurrence of pseudo-pods and changes in the local concentration of F-actin were observed. As a result, it was possible to analyze the temporal-spatial distribution change between pseudo-pod and F-actin. This information is essential in the process of studying the role of F-actin in pseudo-pod formation.

A characteristic phenomenon observed in the analysis using optical flow estimation is that the movement of $\mathrm{F}$-actin does not completely coincide with the formation and deformation of pseudo-pods. Actin flows occur frequently, and some of the flows coincide temporally and spatially with pseudo-pod formation. However, it has a different tendency depending on the pseudo-pod even within a single cell, and the condition was not found in the results of this study. Therefore, further studies are needed to explain the signal transduction pathway of chemotactical motion and pseudo-pod formation.

In this study, I imaged the distribution of actin inside Dicty cells using a 3D-SUPPOSe multi-plane setup and analyzed the data using the new optical flow estimation method. For this measurement, I prepared a device that can measure whole cells in a limited field of view by pressing the cells vertically. The principle of the device has been previously introduced, and in this study, a simple method of manufacturing the device without the need to prepare any microfluidic device was introduced. Through this device, the entire cell could be imaged in a multi-plane setup by limiting the FOV, but the problem remains that the height difference between the boundary and the central region occurs due to the structural limitations of the device. This height difference is induced by the increased chamber size. However, this is not a serious problem since the sample stage can be moved over a large area and the appropriate volume of cells can be selected and imaged from several cells in the chamber.

In the study of dicty cells, the pressure applied vertically to the cells always raises 
doubts about the vitality of the cells. It is difficult to say that pressing the cells can ensure a normal environment, since pushing the cells into a limited volume makes stressful conditions. However, since the shape of dicty cells can be changed, many studies on cell-flattening devices are being conducted, and it has been proven that vitality is maintained through cell movement after applying pressure. For application to other cells, vitality issues must always be considered. On the contrary, if vitality is guaranteed, the application of this device will be possible not only in Dicty cells but also in other cells.

\subsection{Summary}

- For 3D whole-cell measurement, I made a cell flattening device. The device's existing design was copied as it is, but the method to produce was modified to make it easier.

- I imaged the motion of the dicty cell using the deconvolution multiplane setup. Through the discrepancy between COFM and centroid, it was confirmed whether actin flow occurred inside the cell.

- Using the flow estimation method, I analyzed each part of the cell's actin flow for all frames. It was confirmed that the formation of pseudo-pod and actin flow showed temporal inconsistency. 


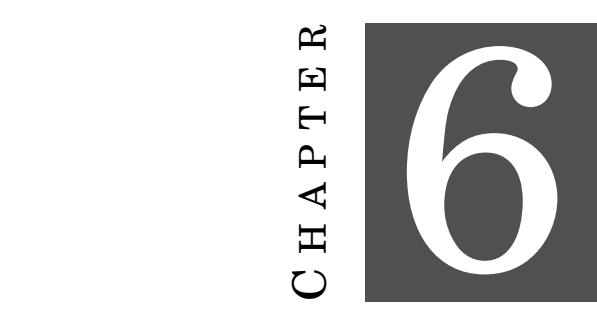

\section{Conclusion}

I

$\mathrm{n}$ this paper, I introduced data analysis methods to improve the performance of multi-plane setup. First, I introduced the intensity calibration method required to reconstruct 3D images. In order to render a 3D image from a multi-plane setup data, the relative intensity between each image plane must be precisely measured. In order to minimize the potential experimental error, a model equation was designed in consideration of the prism structure. Correcting the measured data using this model equation helps to realize a more realistic 3D image by reducing potential errors. An additional method 3.2.1 is also presented to correct some errors that cannot be solved by this method. It is a method to increase the accuracy by maximizing the similarity of the entire image plane by considering the continuity between the image planes. This method can provide the necessary information for more accurate $3 \mathrm{D}$ image reconstruction.

Second, we proposed a new deconvolution method. 3D-SUPPOSe is a modified program of the previously developed SUPPOSe algorithm to be suitable for multi-plane settings. It was changed to control the increasing memory usage for 3D image processing, and the optimization method was changed from a genetic algorithm to a gradient descent algorithm to obtain a more accurate answer. The performance of this algorithm was evaluated by comparing it with other deconvolution methods, and it was also confirmed that the performance was improved by using Fourier Interpolation together.

Interpolation method is very useful to analyze multi-plane setup data. In multi-plane 
setup, the number of image planes that can be measured at one time is limited to eight, and the axial size of a voxel is 6 times larger than the lateral size of it. Interpolation can be used to make the sampling rate uniform in all directions, but this method cannot remove image blur. The most important cause of image blur in multi-plane setup is the missing cone problem of OTF caused by wide-field illumination, and a deconvolution program was used to eliminate it. As the results show, using 3D-SUPPOSe with Fourier interpolation yields the sharpest images with the least artifacts. Therefore, this 3DSUPPOSe was used as a core algorithm for image analysis measured in a deconvolution multi-plane setup.

Finally, an optical flow estimation method is proposed. After reconstructing the image measured in the multi-plane setup into a clear three-dimensional image using 3DSUPPOSe, an algorithm for calculating the movement of fluorescent molecules was proposed. The new optical flow estimation method has a theoretical background similar to 3D-SUPPOSe. In 3D-SUPPOSe, the image is composed through super-positioning of massive virtual emitters. In the resulting image, the intensity in a voxel indicates the number of fluorescent molecules distributed in space, and the movement of the fluorescent signal over time means the flow of the fluorescent molecule. This trend is common to typical fluorescence images, but it becomes clearer by removing the blurring effect. In order to quantify the flow of fluorescent molecules, the optical flow estimation method used a convolution operation in voxel units like 3D-SUPPOSe. This method has the advantage of directly calculating the change in intensity of each voxel, unlike the existing optical flow estimation, which is calculated based on the similarity of small unit patches of the image.

Based on the three proposed methods, cardiomyocytes and dicty cells were studied. First, we analyzed the movement of ACTN-2, which is considered as a marker site in cardiomyocyte studies. Using the deconvolution multi-plane setup, we measured the cell movement at a rate about 30 times faster than the beating motion of the cardiomyocytes. As a result of imaging the distribution of ACTN-2 based on the precisely reconstructed three-dimensional image, the vertical motion of the molecule was visualized. The movement of ACTN-2 which was considered a passive component could be found, but it was not enough to find the biological meaning of the motion. This information will have meaning in future studies on the interaction of various proteins constituting cardiomyocytes, and this motion is expected to provide information related to the regulation of proteins constituting static structures constituting cells. 
Next, in the last chapter I introduced the study of chemotaxis of dicty cells. For 3D whole-cell measurements, I designed a device that can fit cells to the image volume. Although the design of the device is not entirely new, it has suggested a way to make it a little easier. Dicty cells were imaged using this method, and intracellular actin flow was analyzed. By analyzing the flow generated in myosin null cells, I observed the formation and deformation of the actin flow in the process of a pseudo-pod driven chemotactical motion. This observation result shows that a time delay occurs between pseudo-pod formation and F-actin flow. In the future, through a quantitative study on the temporal delay and spatial correlation between the two phenomena, the effect and mechanism of cortical actin flow on pseudo-pod formation can be identified.

The core deconvolution and optical flow estimation methods proposed in this study have a common characteristics of understanding fluorescence images at the molecular level. Both of these programs assume that all fluorescent molecules have the same brightness, and they are dispersed discontinuously. Unit molecules are identical molecules, share the same properties, but have individual motions and are distributed apart from each other in a given space. 3D-SUPPOSe is to describe and optimize the distribution of the unit molecules, and optical flow estimation is an attempt to directly calculate the motion of the molecules.

These algorithms use direct computation methods, and the programs should be improved more. Calculation using 3D-SUPPOSe still requires a long calculation time, and subvoxel calculation has not been solved. Also, there is a need to improve the objective function for calculating noise. The noise removal method should be applied not only to 3D-SUPPOSe but also to the optical flow estimation method. If the newly developed noise removal method is effectively applied to 3D-SUPPOSe and optical flow estimation, the efficiency of these analysis methods will be further increased. Considering the rapidly developing computational technique, it is expected that the problem of computational cost of these analysis methods will be gradually solved.

In this thesis, the newly developed programs were used only for image data analysis of multi-plane setup, and only the advantages as a tool to analyze multi-plane setup datasets were examined. However, these programs have the potential to be widely used for molecular spectroscopy-based image analysis beyond fluorescence microscopy. Since the analysis method needs only the condition that the magnitude of the signal emitted by all molecules is constant, the application possibility is not limited into the 
fluorescence imaging. However, the current super-resolution microscopy technology has been developed in the direction of using the signals and properties of fluorescent molecules. SUPPOSe was developed for the purpose of approximating super-resolution images as presented in the paper,[73] where the necessary condition is that all particles have equal brightness. Therefore, it can be used for all imaging techniques that image the distribution of specific molecules. Therefore, it is expected that the scope of application of these analysis methods will be further expanded in the future. 


\section{BIBLIOGRAPHY}

[1] S. Abrahamsson, H. Blom, A. Agostinho, D. C. Jans, A. Jost, M. Müller, L. Nilsson, K. Bernhem, T. J. Lambert, R. Heintzmann, et AL., Multifocus structured illumination microscopy for fast volumetric super-resolution imaging, Biomedical optics express, 8 (2017), pp. 4135-4140.

[2] S. Abrahamsson, J. Chen, B. HajJ, S. Stallinga, A. Y. Katsov, J. WisNiewski, G. Mizuguchi, P. Soule, F. Mueller, C. D. Darzacq, et Al., Fast multicolor 3d imaging using aberration-corrected multifocus microscopy, Nature methods, 10 (2013), pp. 60-63.

[3] S. Abrahamsson, R. Ilic, J. Wisniewski, B. Mehl, L. Yu, L. Chen, M. DaVANCO, L. OUdJEDI, J.-B. Fiche, B. HAJJ, ET AL., Multifocus microscopy with precise color multi-phase diffractive optics applied in functional neuronal imaging, Biomedical optics express, 7 (2016), pp. 855-869.

[4] S. Abrahamsson, M. McQuilken, S. B. Mehta, A. Verma, J. Larsch, R. Ilic, R. Heintzmann, C. I. Bargmann, A. S. Gladfelter, and R. OldenbourG, Multifocus polarization microscope (mf-polscope) for $3 d$ polarization imaging of up to 25 focal planes simultaneously, Optics express, 23 (2015), pp. 7734-7754.

[5] E. H. Adelson, C. H. Anderson, J. R. Bergen, P. J. Burt, And J. M. Ogden, Pyramid methods in image processing, RCA engineer, 29 (1984), pp. 33-41.

[6] M. AfFolter AND C. J. Weijer, Signaling to cytoskeletal dynamics during chemotaxis, Developmental cell, 9 (2005), pp. 19-34.

[7] D. A. AGARD AND J. W. SEDAT, Three-dimensional architecture of a polytene nucleus, Nature, 302 (1983), pp. 676-681. 
[8] E. Ahmadzadeh, K. Jaferzadeh, S. Shin, And I. Moon, Automated single cardiomyocyte characterization by nucleus extraction from dynamic holographic images using a fully convolutional neural network, Biomedical optics express, 11 (2020), pp. 1501-1516.

[9] F. Amat, E. W. Myers, AND P. J. Keller, Fast and robust optical flow for timelapse microscopy using super-voxels, Bioinformatics, 29 (2013), pp. 373-380.

[10] G. Amselem, M. Theves, A. Bae, E. Bodenschatz, And C. Beta, A stochastic description of dictyostelium chemotaxis, PloS one, 7 (2012), p. e37213.

[11] J. D. ANGRIst AND J.-S. PischKe, Mostly harmless econometrics: An empiricist's companion, Princeton university press, 2008.

[12] Y. Artemenko, K. F. Swaney, And P. N. Devreotes, Assessment of development and chemotaxis in dictyostelium discoideum mutants, in Cell Migration, Springer, 2011, pp. 287-309.

[13] C. Barnes, E. Shechtman, A. Finkelstein, And D. B. Goldman, Patchmatch: A randomized correspondence algorithm for structural image editing, ACM Trans. Graph., 28 (2009), p. 24.

[14] S. S. BEAUCHEMIN AND J. L. BARRON, The computation of optical flow, ACM computing surveys (CSUR), 27 (1995), pp. 433-466.

[15] M. Bertero, P. Boccacci, G. Desiderà, AND G. Vicidomini, Image deblurring with poisson data: from cells to galaxies, Inverse Problems, 25 (2009), p. 123006.

[16] E. Betzig, G. H. Patterson, R. Sougrat, O. W. Lindwasser, S. Olenych, J. S. Bonifacino, M. W. Davidson, J. Lippincott-Schwartz, And H. F. HESS, Imaging intracellular fluorescent proteins at nanometer resolution, Science, 313 (2006), pp. 1642-1645.

[17] D. S. BIGGS, 3d deconvolution microscopy, Current Protocols in Cytometry, 52 (2010), pp. 12-19.

[18] M. Born AND E. Wolf, Principles of optics, Elsevier, 2013.

[19] T. Bretschneider, S. Diez, K. Anderson, J. Heuser, M. Clarke, A. Müller-Taubenberger, J. Köhler, And G. Gerisch, Dynamic actin 
patterns and arp2 / 3 assembly at the substrate-attached surface of motile cells, Current Biology, 14 (2004), pp. 1-10.

[20] T. Brox, A. Bruhn, N. PAPenberG, And J. Weickert, High accuracy optical flow estimation based on a theory for warping, in European conference on computer vision, Springer, 2004, pp. 25-36.

[21] T. BRox AND J. MALIK, Large displacement optical flow: descriptor matching in variational motion estimation, IEEE transactions on pattern analysis and machine intelligence, 33 (2010), pp. 500-513.

[22] P. Camelliti, J. O. Gallagher, P. Kohl, and A. D. McCulloch, Micropatterned cell cultures on elastic membranes as an in vitro model of myocardium, Nature Protocols, 1 (2006), pp. 1379-1391.

[23] Y. Chen, Y. C. EldAR, AND A. J. Goldsmith, Shannon meets nyquist: capacity limits of sampled analog channels, in 2011 IEEE International Conference on Acoustics, Speech and Signal Processing (ICASSP), IEEE, 2011, pp. 3104-3107.

[24] J.-A. Conchello AND J. G. MCNAlly, Fast regularization technique for expectation maximization algorithm for optical sectioning microscopy, in ThreeDimensional Microscopy: Image Acquisition and Processing III, vol. 2655, International Society for Optics and Photonics, 1996, pp. 199-209.

[25] C. CREMER AND T. CREMER, Considerations on a laser-scanning-microscope with high resolution and depth of field, Microscopica acta, (1974), pp. 31-44.

[26] H. B. CURRY, The method of steepest descent for non-linear minimization problems, Quarterly of Applied Mathematics, 2 (1944), pp. 258-261.

[27] M. DAVEnPoRT, The fundamentals of compressive sensing, IEEE Signal Processing Society Online Tutorial Library, 12 (2013).

[28] M. W. Davidson, Education in microscopy and digital imaging, 1999.

[29] M. W. Davidson, Understanding digital imaging, 1999.

[30] E. N. Dedkova AND L. A. Blatter, Calcium signaling in cardiac mitochondria, Journal of molecular and cellular cardiology, 58 (2013), pp. 125-133. 
[31] T. Dertinger, R. Colyer, G. Iyer, S. Weiss, and J. Enderlein, Fast, background-free, $3 d$ super-resolution optical fluctuation imaging (sofi), Proceedings of the National Academy of Sciences, 106 (2009), pp. 22287-22292.

[32] A. Descloux, K. Grussmayer, E. Bostan, t. Lukes, A. Bouwens, A. Sharipov, S. Geissbuehler, A.-L. Mahul-Mellier, H. Lashuel, M. LeUteneGGer, ET AL., Combined multi-plane phase retrieval and superresolution optical fluctuation imaging for $4 D$ cell microscopy, Nature Photonics, 12 (2018), pp. 165-172.

[33] A. Descloux, M. Müller, V. Navikas, A. Markwirth, R. Van den Eynde, T. Lukes, W. Hübner, T. Lasser, A. Radenovic, P. Dedecker, et Al., High-speed multiplane structured illumination microscopy of living cells using an image-splitting prism, Nanophotonics, 9 (2019), pp. 143-148.

[34] S. Diez, G. Gerisch, K. Anderson, A. Müller-Taubenberger, and T. BRETSCHNEIDER, Subsecond reorganization of the actin network in cell motility and chemotaxis, Proceedings of the National Academy of Sciences, 102 (2005), pp. 7601-7606.

[35] D. L. Donoho, For most large underdetermined systems of linear equations the minimal l1-norm solution is also the sparsest solution, Communications on Pure and Applied Mathematics: A Journal Issued by the Courant Institute of Mathematical Sciences, 59 (2006), pp. 797-829.

[36] T. T. Egelhoff, D. J. Manstein, And J. A. Spudich, Complementation of myosin null mutants in dictyostelium discoideum by direct functional selection, Developmental biology, 137 (1990), pp. 359-367.

[37] E. EHLER, Actin-associated proteins and cardiomyopathy,Äîthe ,Äòunknown,Äôbeyond troponin and tropomyosin, Biophysical reviews, 10 (2018), pp. 1121-1128.

[38] A. E. Eiben, P.-E. RAUe, AND Z. RUtTKAY, Genetic algorithms with multi-parent recombination, in International conference on parallel problem solving from nature, Springer, 1994, pp. 78-87.

[39] S. Eliott, G. H. Joss, A. Spudich, and K. L. Williams, Patterns in dictyostelium discoideum: the role of myosin ii in the transition from the unicellular to the multicellular phase, Journal of Cell Science, 104 (1993), pp. 457-466. 
[40] G. FAulkner, G. LANFranchi, AND G. VAlle, Telethonin and other new proteins of the z-disc of skeletal muscle, IUBMB life, 51 (2001), pp. 275-282.

[41] D. Frank, C. Kuhn, H. A. KATUs, And N. Frey, The sarcomeric $z$-disc: a nodal point in signalling and disease, Journal of molecular medicine, 84 (2006), p. 446.

[42] F. Galton, Kinship and correlation, The North American Review, 150 (1890), pp. 419-431.

[43] C. F. GAUss, Theoria combinationis observationum erroribus minimis obnoxiae, vol. 2, H. Dieterich, 1823.

[44] S. Geissbuehler, A. Sharipov, A. Godinat, N. L. Bocchio, P. A. Sandoz, A. Huss, N. A. Jensen, S. Jakobs, J. Enderlein, F. G. Van Der Goot, ET AL., Live-cell multiplane three-dimensional super-resolution optical fluctuation imaging, Nature Communications, 5 (2014), pp. 1-7.

[45] G. Gerisch, Chemotaxis in dictyostelium, Annual Review of Physiology, 44 (1982), pp. $535-552$.

[46] J. J. GiBSON, The perception of the visual world., (1950).

[47] J. R. GisPert, Coordination chemistry, vol. 483, Wiley-VCH Weinheim, 2008.

[48] C. Grădinaru, J. M. Łopacińska, J. Huth, H. A. Kestler, H. FlyvbJerg, AND K. MøLHAVE, Assessment of automated analyses of cell migration on flat and nanostructured surfaces, Computational and structural biotechnology journal, 1 (2012), p. e201207004.

[49] J. M. GuerRA, Super-resolution through illumination by diffraction-born evanescent waves, Applied physics letters, 66 (1995), pp. 3555-3557.

[50] M. Guo, Y. Li, Y. Su, T. Lambert, D. Dalle Nogare, M. W. Moyle, L. H. Duncan, R. IKegami, A. SAntella, I. ReY-SuAREZ, ET AL., Rapid image deconvolution and multiview fusion for optical microscopy, Nature Biotechnology, (2020), pp. 1-10.

[51] B. Hebert, S. Costantino, and P. W. Wiseman, Spatiotemporal image correlation spectroscopy (stics) theory, verification, and application to protein velocity mapping in living cho cells, Biophysical journal, 88 (2005), pp. 3601-3614. 
[52] S. W. Hell, S. Lindek, C. Cremer, and E. H. Stelzer, Measurement of the 4piconfocal point spread function proves $75 \mathrm{~nm}$ axial resolution, Applied Physics Letters, 64 (1994), pp. 1335-1337.

[53] S. W. Hell and J. Wichmann, Breaking the diffraction resolution limit by stimulated emission: stimulated-emission-depletion fluorescence microscopy, Optics Letters, 19 (1994), pp. 780-782.

[54] C. A. Henderson, C. G. Gomez, S. M. Novak, L. Mi-Mi, and C. C. GregoRIO, Overview of the muscle cytoskeleton, Comprehensive Physiology, 7 (2011), pp. 891-944.

[55] B. K. HoRn And B. G. Schunck, Determining optical flow, Artificial intelligence, 17 (1981), pp. 185-203.

[56] Y. HU, R. SonG, AND Y. LI, Efficient coarse-to-fine patchmatch for large displacement optical flow, in Proceedings of the IEEE Conference on Computer Vision and Pattern Recognition, 2016, pp. 5704-5712.

[57] S. Hugelier, J. J. De Rooi, R. Bernex, S. Duwé, O. Devos, M. Sliwa, P. Dedecker, P. H. Eilers, AND C. Ruckebusch, Sparse deconvolution of high-density super-resolution images, Scientific Reports, 6 (2016), p. 21413.

[58] S. Hugelier, P. Eilers, O. Devos, And C. Ruckebusch, Improved superresolution microscopy imaging by sparse deconvolution with an interframe penalty, Journal of Chemometrics, 31 (2017), p. e2847.

[59] H. Ikoma, M. Broxton, T. Kudo, And G. Wetzstein, A convex 3D deconvolution algorithm for low photon count fluorescence imaging, Scientific Reports, 8 (2018), pp. 1-12.

[60] M. S. Itano, M. Bleck, D. S. Johnson, And S. M. Simon, Readily accessible multiplane microscopy: $3 d$ tracking the hiv-1 genome in living cells, Traffic, 17 (2016), pp. 179-186.

[61] Y. Iwadate, C. OKimura, K. Sato, Y. Nakashima, M. Tsujioka, and K. MiNAMI, Myosin-ii-mediated directional migration of dictyostelium cells in response to cyclic stretching of substratum, Biophysical journal, 104 (2013), pp. $748-758$. 
[62] J. S. KING AND R. H. INSALL, Chemotaxis: finding the way forward with dictyostelium, Trends in cell biology, 19 (2009), pp. 523-530.

[63] D. P. KIngma AND J. BA, Adam: A method for stochastic optimization, arXiv preprint arXiv:1412.6980, (2014).

[64] A. M. Lacapmesure, S. Martínez, and O. E. Martínez, A new objective function for super-resolution deconvolution of microscopy images by means of a genetic algorithm, in Proceedings of the 2020 Genetic and Evolutionary Computation Conference Companion, 2020, pp. 271-272.

[65] A. M. LEGEndRE, Nouvelles méthodes pour la détermination des orbites des comètes: avec un supplément contenant divers perfectionnemens de ces méthodes et leur application aux deux comètes de 1805, Courcier, 1806.

[66] C. LemarÉchal, Cauchy and the gradient method, Doc Math Extra, 251 (2012), p. 10.

[67] L. B. LUCY, An iterative technique for the rectification of observed distributions, The astronomical journal, 79 (1974), p. 745.

[68] J. MACIEL AND J. P. Costeira, A global solution to sparse correspondence problems, IEEE Transactions on Pattern Analysis and Machine Intelligence, 25 (2003), pp. 187-199.

[69] B. F. MADORE AND W. L. FREEDMAN, Super-nyquist sampling strategies, in Astronomical Data Analysis II, vol. 4847, International Society for Optics and Photonics, 2002, pp. 156-166.

[70] V. Maltsev, H. Sabbah, M. Tanimura, M. Lesch, S. Goldstein, And A. UnDROVINAS, Relationship between action potential, contraction-relaxation pattern, and intracellular ca2+ transient in cardiomyocytes of dogs with chronic heart failure, Cellular and Molecular Life Sciences CMLS, 54 (1998), pp. 597605 .

[71] S. Manandhar, P. Bouthemy, E. Welf, G. Danuser, P. Roudot, and C. KERVRANN, $3 d$ flow field estimation and assessment for live cell fluorescence microscopy, Bioinformatics, 36 (2020), pp. 1317-1325. 
[72] B. Mandracchia, X. Hua, C. Guo, J. Son, T. Urner, and S. Jia, Fast and accurate scmos noise correction for fluorescence microscopy, Nature Communications, 11 (2020), pp. 1-12.

[73] S. Martinez, M. Toscani, ANd O. E. Martinez, Superresolution method for a single wide-field image deconvolution by superposition of point sources, Journal of Microscopy, 275 (2019), pp. 51-65.

[74] J. G. McNally, T. Karpova, J. Cooper, and J. A. Conchello, Threedimensional imaging by deconvolution microscopy, Methods, 19 (1999), pp. 373385 .

[75] E. MÉmin ANd P. PÉrez, Hierarchical estimation and segmentation of dense motion fields, International Journal of Computer Vision, 46 (2002), pp. 129155.

[76] R. Milo AND R. Phillips, Cell biology by the numbers, Garland Science, 2015.

[77] J. Min, C. Vonesch, H. Kirshner, L. Carlini, N. Olivier, S. Holden, S. MANLEY, J. C. Ye, AND M. UnSER, Falcon: fast and unbiased reconstruction of high-density super-resolution microscopy data, Scientific reports, 4 (2014), p. 4577.

[78] A. C. Murphy AND P. W. Young, The actinin family of actin cross-linking proteins-a genetic perspective, Cell \& bioscience, 5 (2015), pp. 1-9.

[79] D. MurPhy And M. Davidson, Fundamentals of light microscopy and electronic imaging, secondwiley, 2013.

[80] K. P. MURPhy, Machine learning: a probabilistic perspective, MIT press, 2012.

[81] A. NEICE, Methods and limitations of subwavelength imaging, Advances in imaging and electron physics, 163 (2010), pp. 117-140.

[82] T. Oda AND H. YANAGISAWA, Cryo-electron tomography of cardiac myofibrils reveals a $3 d$ lattice spring within the $z$-discs, Communications biology, 3 (2020), pp. 1-9.

[83] L. Oudjedi, J.-B. Fiche, S. Abrahamsson, L. Mazenq, A. Lecestre, P.-F. Calmon, A. Cerf, and M. Nöllmann, Astigmatic multifocus microscopy 
enables deep 3d super-resolved imaging, Biomedical optics express, 7 (2016), pp. 2163-2173.

[84] M. Pharr, W. JAKOB, AND G. Humphreys, Physically based rendering: From theory to implementation, Morgan Kaufmann, 2016.

[85] S. PINEDA SAN JUAN, Statistical methods for the integration analysis of-omics data (genomics, epigenomics and transcriptomics): An application to bladder cancer, PhD thesis, Université de Liège,,Äã Liège,,Äã,Äã Belgique, 2015.

[86] J. Pinero AND D. BATES, Mixed-effects models in s and s-plus (statistics and computing), 2000.

[87] P. Prabhat, S. Ram, E. S. WARD, And R. J. Ober, Simultaneous imaging of different focal planes in fluorescence microscopy for the study of cellular dynamics in three dimensions, IEEE transactions on nanobioscience, 3 (2004), pp. 237-242.

[88] W. G. Pyle AND R. J. Solaro, At the crossroads of myocardial signaling: the role of z-discs in intracellular signaling and cardiac function, Circulation research, 94 (2004), pp. 296-305.

[89] A. J. Ribeiro, Y.-S. Ang, J.-D. Fu, R. N. Rivas, T. M. Mohamed, G. C. Higgs, D. SRivastava, AND B. L. PRUitT, Contractility of single cardiomyocytes differentiated from pluripotent stem cells depends on physiological shape and substrate stiffness, Proceedings of the National Academy of Sciences, 112 (2015), pp. $12705-12710$.

[90] J. E. A. Ribeiro, N. Pinotsis, A. Ghisleni, A. Salmazo, P. V. Konarev, J. Kostan, B. Sjöblom, C. Schreiner, A. A. Polyansky, E. A. GkougKOULIA, ET AL., The structure and regulation of human muscle $\alpha$-actinin., Cell, 159 (2014), pp. 1447-1460.

[91] W. H. RICHARDSON, Bayesian-based iterative method of image restoration, JOSA, 62 (1972), pp. 55-59.

[92] D. E. Rumelhart, G. E. Hinton, AND R. J. Williams, Learning representations by back-propagating errors, nature, 323 (1986), pp. 533-536. 
[93] M. J. RUSt, M. BATES, AND X. ZhUANG, Sub-diffraction-limit imaging by stochastic optical reconstruction microscopy (storm), Nature methods, 3 (2006), pp. 793796.

[94] D. Sage, L. Donati, F. Soulez, D. Fortun, G. Schmit, A. Seitz, R. Guiet, C. Vonesch, AND M. Unser, Deconvolutionlab2: An open-source software for deconvolution microscopy, Methods, 115 (2017), pp. 28-41.

[95] J. M. SANGER AND J. W. SANGER, The dynamic $Z$ bands of striated muscle cells, Science Signaling, 1 (2008), pp. pe37-pe37.

[96] J. W. Sanger, J. Wang, Y. Fan, J. White, L. Mi-Mi, D. K. Dube, J. M. Sanger, AND D. PRUYNE, Assembly and maintenance of myofibrils in striated muscle, in The Actin Cytoskeleton, Springer, 2016, pp. 39-75.

[97] E. Schäfer, M. Tarantola, E. Polo, C. Westendorf, N. Oikawa, E. BodenSCHATZ, B. GEIL, AND A. JANSHOFF, Chemotaxis of dictyostelium discoideum: Collective oscillation of cellular contacts, PLoS One, 8 (2013), p. e54172.

[98] A. SCHRIJVER, On the history of combinatorial optimization (till 1960), Handbooks in operations research and management science, 12 (2005), pp. 1-68.

[99] J.-B. Sibarita, Deconvolution microscopy, in Microscopy Techniques, Springer, 2005, pp. 201-243.

[100] B. Sjöвlom, A. SAlmazo, And K. Djinović-CARUgo, $\alpha$-actinin structure and regulation, Cellular and molecular life sciences, 65 (2008), pp. 2688-2701.

[101] S. C. Stein, A. Huss, D. Hähnel, I. Gregor, AND J. Enderlein, Fourier interpolation stochastic optical fluctuation imaging, Optics Express, 23 (2015), pp. 16154-16163.

[102] F. STRÖHL, Single-shot volumetric microscopy, Imaging and microscopy, (2020), p. 24.

[103] P. Thevenaz, U. E. Ruttimann, And M. Unser, A pyramid approach to subpixel registration based on intensity, IEEE Transactions on Image Processing, 7 (1998), pp. 27-41. 
[104] M. Tiburcy, J. E. Hudson, P. Balfanz, S. Schlick, T. Meyer, M.-L. Chang Liao, E. Levent, F. RaAd, S. Zeidler, E. Wingender, et Al., Defined engineered human myocardium with advanced maturation for applications in heart failure modeling and repair, Circulation, 135 (2017), pp. 18321847.

[105] H. C. Tie, B. Chen, X. Sun, L. Cheng, And L. Lu, Quantitative localization of a Golgi protein by imaging its center of fluorescence mass, JoVE (Journal of Visualized Experiments), (2017), p. e55996.

[106] A. N. Tikhonov, A. Goncharsky, V. Stepanov, and A. G. Yagola, Numerical methods for the solution of ill-posed problems, vol. 328, Springer Science \& Business Media, 2013.

[107] J. TOlles AND W. J. MEURER, Logistic regression: relating patient characteristics to outcomes, Jama, 316 (2016), pp. 533-534.

[108] E. Toprak, H. Balci, B. H. Blehm, AND P. R. Selvin, Three-dimensional particle tracking via bifocal imaging, Nano letters, 7 (2007), pp. 2043-2045.

[109] H. Trevor, T. RoberT, AND F. JH, The elements of statistical learning: data mining, inference, and prediction, 2009.

[110] M. A. Unger, H.-P. Chou, T. Thorsen, A. Scherer, And S. R. QuAKe, Monolithic microfabricated valves and pumps by multilayer soft lithography, Science, 288 (2000), pp. 113-116.

[111] G. D. B. Vazquez, A. M. Lacapmesure, M. Toscani, S. Martínez, and O. E. MARTÍNEZ, Super-resolution microscopy from standard images, Optics and Photonics News, 31 (2020), pp. 58-58.

[112] G. D. B. Vazquez, S. Martínez, and O. E. Martínez, Super-resolved edge detection in optical microscopy images by superposition of virtual point sources, Optics Express, 28 (2020), pp. 25319-25334.

[113] B. J. WALKER AND R. J. WHEELER, High-speed multifocal plane fluorescence microscopy for three-dimensional visualisation of beating flagella, Journal of cell science, 132 (2019). 
[114] Y. WaN, K. MCDole, AND P. J. Keller, Light-sheet microscopy and its potential for understanding developmental processes, Annual review of cell and developmental biology, 35 (2019), pp. 655-681.

[115] C. Westendorf, A. J. Bae, C. Erlenkamper, E. Galland, C. Franck, E. BoDENSCHATZ, AND C. BETA, Live cell flattening,Äîtraditional and novel approaches, PMC biophysics, 3 (2010), p. 9.

[116] N. WIENER, Extrapolation, interpolation, and smoothing of stationary time series: with engineering applications, (1949).

[117] A. T. R. Williams, An introduction to fluorescence microscopy, 2000.

[118] F. Xu, R. Bierman, F. Healy, ANd H. NGUYen, A multi-scale model of escherichia coli chemotaxis from intracellular signaling pathway to motility and nutrient uptake in nutrient gradient and isotropic fluid environments, Computers \& Mathematics with Applications, 71 (2016), pp. 2466-2478.

[119] F. W. Yang, C. Venkataraman, V. Styles, V. Kuttenberger, E. Horn, Z. VON GutTENBerg, AND A. MADZvamuse, A computational framework for particle and whole cell tracking applied to a real biological dataset, Journal of biomechanics, 49 (2016), pp. 1290-1304.

[120] R. D. Yates AND D. J. Goodman, Probability and stochastic processes: a friendly introduction for electrical and computer engineers, John Wiley \& Sons, 2014.

[121] S. Yumura, G. Itoh, Y. Kikuta, T. KikUChI, T. Kitanishi-Yumura, AND M. TsujIOKA, Cell-scale dynamic recycling and cortical flow of the actinmyosin cytoskeleton for rapid cell migration, Biology open, 2 (2013), pp. 200209 .

[122] L. Zhu, W. Zhang, D. Elnatan, AND B. Huang, Faster STORM using compressed sensing, Nature Methods, 9 (2012), pp. 721-723. 


\section{ACKNOWLEDGements}

This opportunity for my doctoral degree was precious to me. I appreciate everybody who gave me this opportunity and helped that I could make it.

First of all, I'd like to thank my supervisor, prof. Joerg Enderlein, for giving me this interesting research project, and this opportunity to get $\mathrm{PhD}$ degree. I'd like to thank the other thesis advisory committee members, prof. Andreas Janshoff and prof. Alexander Egner, who understood my complex situation and helped me successfully complete my doctoral program.

I thank Dr. Ingo Gregor for his help with my thesis correction and lots of things in the laboratory. I'd like to thank Alexey, Anna, Roman, Oleksii, Subhabrata, Sebastian, Arindam, Christoph, Shun, Soheil, Steffen, Tao, Nazar, Akshita, and Aditya, who has been in the same space for a long time.

I would also like to thank Sangeun for being by my side and overcoming together the difficult moments. I appreciate the support of my parents and all family members.

Lastly, I acknowledge the financial support of this work by SFB 937 and MBExC. 